\title{
Maximal Hypersurfaces in Asymptotically Stationary Spacetimes
}

\author{
Piotr T. Chruściell \\ Max Planck Institut für Astrophysik \\ Karl Schwarzschild Strasse 1 \\ D 8046 Garching bei München \\ Robert M. Wald \\ Enrico Fermi Institute and Department of Physics \\ University of Chicago \\ 5640 S. Ellis Ave., Chicago, IL 60637.
}

\begin{abstract}
Existence of maximal hypersurfaces and of foliations by maximal hypersurfaces is proven in two classes of asymptotically flat spacetimes which possess a one parameter group of isometries whose orbits are timelike "near infinity". The first class consists of strongly causal asymptotically flat spacetimes which contain no "black hole or white hole" (but may contain "ergoregions" where the Killing orbits fail to be timelike). The second class of spacetimes possess a black hole and a white hole, with the black and white hole horizons intersecting in a compact 2-surface $S$.
\end{abstract}

\footnotetext{
${ }^{*}$ Alexander von Humboldt fellow. On leave of absence from the Institute of Mathematics, Polish Academy of Sciences, Warsaw. E-mail: piotr@ibm-1.mpa.ipp-garching.mpg.de. Supported in part by KBN grant \#2 1047 9101.

${ }^{\dagger}$ Supported in part by NSF grant PHY-8918388. E-mail: rmwa@midway.uchicago.edu.
} 


\section{Introduction}

The question of the existence of maximal slices (i.e., slices with vanishing trace, $K=K^{a}{ }_{a}$ of extrinsic curvature, $K_{a b}$ ) in asymptotically flat spacetimes has arisen frequently in the analysis of many issues in general relativity. The main reason for this is that the "kinetic term"

$$
K^{a b} K_{a b}-K^{2}
$$

in the Hamiltonian constraint equation is non-negative when $K=0$, thereby simplifying many arguments. Another reason is that the momentum constraint becomes conformally invariant when $K=0$.

The issue of the existence of maximal hypersurfaces arose again recently in an analysis of solutions to the Einstein-Yang-Mills equation by Sudarsky and Wald [21]. In theorems 3.3, 3.4, and the discussion following theorem 3.4 of [21] two results were proven, which may be roughly summarized as follows ( $c f$. [21] for precise statements of the asymptotic conditions assumed):

1. Consider an asymptotically flat (with a single "end") solution to the Einstein-Yang-Mills equations (on a trivial $S U(2)$ principal bundle) with a Killing vector field $X$, timelike at infinity, which has vanishing electric charge or asymptotically vanishing electrostatic potential. Suppose there exists an asymptotically flat maximal slice $\Sigma$ with compact interior (i.e., a two-sphere in the asymptotic region bounds a compact subset of $\Sigma$ ), such that $X$ is asymptotically orthogonal to $\Sigma$ (i.e., $X \longrightarrow_{r \rightarrow \infty} \partial / \partial t$ in the asymptotically Minkowskian coordinates in which $\Sigma \subset\{t=0\}$ ). Then the solution is static (i.e., $X$ is orthogonal to $\Sigma$ ) and has vanishing Yang-Mills electric field on the static hypersurfaces.)

2. Consider an asymptotically flat solution to the Einstein-Yang-Mills equations (on a trivial $S U(2)$ principal bundle) which possesses a Killing vector field $X$ which is timelike at infinity and possesses a black and white hole, whose horizons comprise a bifurcate Killing horizon with bifurcation surface $S$. Suppose that $X$ is normal to the horizon (i.e., suppose that the angular velocity of the horizon vanishes) and suppose that the solution has vanishing electric charge or asymptotically vanishing electrostatic potential. Suppose further that there exists an asymptotically flat maximal hypersurface $\Sigma$ with boundary $S$, and compact interior such that $X$ is asymptotically orthogonal to $\Sigma$. Then the solution is static "in the exterior world" (i.e., $X$ is orthogonal to $\Sigma$ ), with $X$ strictly timelike outside of the black and white holes, and has vanishing electric field on the static hypersurfaces. (In the Einstein-Maxwell (on a trivial $U(1)$ principal bundle) case, the hypothesis that the electric charge or the asymptotic electrostatic potential vanishes may be dropped, and it then can be shown that the solution is static in the exterior world and has vanishing magnetic field on the static hypersurfaces.)

Clearly, to obtain a more satisfactory picture one has to understand how restrictive are the conditions of existence of maximal hypersurfaces made above. In the past decade, some progress has been made in proving existence of maximal slices in asymptotically flat spacetimes [2], [5]. In particular, it has been shown that asymptotically flat maximal slices always exist in strictly stationary, asymptotically flat spacetimes [5]. Unfortunately, this theorem requires that the Killing vector field be timelike everywhere, not just "near infinity". Furthermore, this theorem 
does not encompass the situation where one wishes the maximal hypersurface to pass through a given 2-surface (e.g. the bifurcation 2-surface, $S$, of the second result above).

The purpose of this paper is to extend the previous results on existence of maximal hypersurfaces to encompass the situations considered by Sudarsky and Wald, and, in fact, some more general situations as well. We will prove that in asymptotically stationary spacetimes, there exist maximal hypersurfaces of the type needed in their results 1 and 2 above. Indeed, our analysis will be more general in that

1. Einstein's equation will not be used (i.e., no energy conditions will be imposed), and

2. we will assume weaker asymptotic conditions on our spacetimes than assumed in 21].

In addition, in an appendix we shall generalize our results to "asymptotically stationaryrotating spacetimes". Thus, under the conditions of this paper, the hypotheses of the existence of a maximal hypersurface can be removed from theorems 3.3 and 3.4 of [21] (as well as from the discussion in [21] following theorem 3.4).

We refer the reader to section 2 below for a precise specification of the classes of spacetimes considered in our paper and we refer the reader to section 0 for the precise statement (and proof) of our existence theorems on maximal slices. However, it is worth elucidating here by some informal discussion and examples the nature of the spacetimes to which our results apply - as well as those which are not treated by our analysis.

To begin, we emphasize that we restrict attention to strongly causal spacetimes $\left(M, g_{a b}\right)$ for which there exists a smooth, acausal hypersurface $\Sigma \subset M$ (possibly possessing a compact boundary $S$ ) with a finite number of asymptotically flat "ends", $\Sigma_{i}$. Furthermore, we require $\left(M, g_{a b}\right)$ to possess a one-parameter group of isometries which has timelike orbits at each "end". (As mentioned above, a generalization to the case where $X$ is "stationary-rotating" (rather than timelike) at each end is given in the appendix.) We shall denote the Killing field which generates these isometries as $X$ and shall denote the isometry corresponding to parameter $t$ by $\phi[X]_{t}$. The orbit of $\Sigma_{i}$ under $\phi[X]_{t}$ will be denoted as $M_{i}$. We emphasize that we do not impose any conditions on the Killing orbits in the "interior portion" of the spacetime. In particular, "ergoregions" where $X$ is null or spacelike are permitted.

The general form of the spacetime metric in the spacetimes we consider can be described in concrete terms as follows. In section 1 we shall show that in the class of spacetimes which we consider, there always exists a smooth, spacelike hypersurface $\Sigma^{\prime}$ such that $X$ is transverse to $\Sigma^{\prime}$, and the orbits of $X$ passing through $\Sigma^{\prime}$ are diffeomorphic to $\mathbb{R}$. We define the set $M_{\Sigma^{\prime}}=\left\{p \in M: \exists t \in \mathbb{R}\right.$ such that $\left.\phi[X]_{t}(p) \in \Sigma^{\prime}\right\}$. Then $M_{\Sigma^{\prime}}$ is an open submanifold of $M$ diffeomorphic to $\Sigma^{\prime} \times \mathbb{R}$. Furthermore, the hypersurface $\Sigma^{\prime}$ is given by the equation $t=0$, where $t$ is the function on $M_{\Sigma^{\prime}}$ appearing in the definition of $M_{\Sigma^{\prime}}$. It follows that on $M_{\Sigma^{\prime}}$ the metric can be written in the form

$$
\begin{aligned}
& g_{\mu \nu} d x^{\mu} d x^{\nu}=\left(|\beta|_{\gamma}^{2}-\alpha\right) d t^{2}+2 \beta d t+\gamma \\
& X^{\mu} \partial_{\mu}=\partial_{t}, \quad \frac{\partial \alpha}{\partial t}=\frac{\partial \beta}{\partial t}=\frac{\partial \gamma}{\partial t}=0
\end{aligned}
$$

where $\alpha>0$ is a function on $\Sigma^{\prime}, \beta$ is a one form on $\Sigma^{\prime}$, and $\gamma$ is a Riemannian metric on $\Sigma^{\prime}$. Furthermore, we have,

$$
g_{a b} X^{a} X^{b} * 0 \Longleftrightarrow-\alpha+|\beta|_{\gamma}^{2} * 0
$$


where $*$ stands for $=,>$ or $<$. It should be stressed that (1.1) defines a Lorentzian metric regardless of the sign of $g_{a b} X^{a} X^{b}$ provided only that $\alpha>0$ and that $\gamma$ is a Riemannian metric. When $X$ is spacelike, then all the coordinate vectors $\frac{\partial}{\partial x^{\mu}}, \mu=0, \ldots, 3$ are spacelike and one loses the a priori control of the slopes of the light cones, which is at the origin of the following difficulty: From the point of view of PDE theory, when considering existence of maximal hypersurfaces a natural hypothesis is that of compactness of domains of dependence of compact sets. Given a metric of the form (1.1) in which $g_{a b} X^{a} X^{b}$ is allowed to change sign, it seems far from being a trivial exercise to determine whether or not compactness of domains of dependence of compact sets holds. Nevertheless, in Section 3 we shall show that this compactness property holds for the spacetimes we consider.

Since the spacetimes we consider possess a hypersurface $\Sigma$ which extends to one or more asymptotically flat "ends", $\Sigma_{i}$ (with orbits $M_{i}$ under $\phi[X]_{t}$ ), the notions of a "black hole" and a "white hole" can be introduced. In this paper, we define a the black hole region of the spacetime to consist of the events which do not lie in the past of the union of the asymptotically flat regions, $M_{i}$. This notion of black hole should be sharply contrasted with the set of events which merely fail to lie in the past of a particular region $M_{i}$. (This set of events will be referred to as the black hole with respect to $M_{i}$ ). The notions of a white hole and a white hole with respect to $M_{i}$ are defined similarly, with "past" replaced by "future". Note that it is possible for a spacetime to possess a black and/or white hole with respect to one or more individual, $M_{i}$, and yet fail to have a black hole and/or white hole; indeed, the spacetime composed of the union of blocks $B, D$, and $E$ of Figure 1.1 provides an example of such a spacetime. Note also that if more than one asymptotic region is present in the spacetime, the definition of the black and white hole regions may depend upon the choice of $\Sigma$, since only the asymptotic regions, $M_{i}$, generated by ends, $\Sigma_{i}$, of $\Sigma$ enter the definition. Thus, for example, in the spacetime of Figure 1.1 below, the black hole region for slice $\Sigma$ is block $F$, but the black hole region for slice $\Sigma^{\prime}$ is the union of blocks $D, E$, and $F$, and the black hole region for hypersurface $\Sigma^{\prime \prime}$ is the union of blocks $C, D, E$, and $F$.

The spacetimes to which our theorems apply divide into the following two classest: (1) $\Sigma$ is a slice (i.e., a closed hypersurface without boundary) whose "interior portion" is compact, and no black hole or white hole is present. In this case theorem 4.1 establishes existence of a maximal slice which is complete, asymptotically flat at each end and is asymptotically orthogonal to $X$. (2) $\Sigma$ is a hypersurface with compact boundary $S$ (which need not be connected) and the "interior portion" of $\Sigma$ is compact. The spacetime contains both a black hole and a white hole and their horizons intersect at $S$. In this case, Theorem 4.2 establishes existence of a maximal hypersurface with boundary $S$, which is complete, asymptotically flat at the ends and is asymptotically orthogonal to $X$.

It should be emphasized, that our results on existence of maximal surfaces are sharp, in the following sense: There exist asymptotically flat space-times which contain a black hole region, and a white hole region, and in which no complete, asymptotically flat, maximal surfaces which are asymptotically orthogonal to the Killing vector exist. An examplea of such a space-time is presented at the beginning of Section 1 .

\footnotetext{
${ }^{1}$ The reader should note that the classes of space-times (1) and (2) discussed here correspond to the classes of space-times (a) and (b) defined in the main body of the paper with additional conditions imposed upon the black and white hole regions of the spacetime.

${ }^{2}$ This example was suggested to us by Dieter Brill. We wish to thank him for useful discussions concerning the question of sharpness of Theorem 4.1.
} 
We now give some examples of spacetimes which lie in these classes, as well as some examples which do not. First, class (1) includes all asymptotically flat spacetimes possessing an asymptotically flat slice, $\Sigma$, with compact interior, such that $X$ is globally timelike. (Namely, if $X$ is globally timelike, it is easily seen that no black hole or white hole can be present: The event horizon of a black or white hole is a null surface which is mapped into itself by the isometries, so if a black or white hole exists, $X$ must be null or spacelike on its horizon.) Numerous examples of such spacetimes are easily constructed using (1.1). Existence of maximal slices when $X$ is globally timelike was previously proven in [5]. Thus, Theorem 4.1 may be viewed as a generalization of this result to the case where an "ergoregion" (but no black or white hole) is present.

An explicit example of a spacetime in class (1) which contains an ergoregion may be constructed as follows. Let $g_{a b}$ be a stationary, axisymmetric metric on $\mathbb{R}^{4}$ of the form,

$$
d s^{2}=-V d t^{2}+2 W d t d \varphi+r^{2} \sin ^{2} \theta d \varphi^{2}+r^{2} d \theta^{2}+d r^{2} .
$$

Choose $r_{1}, r_{2}, \theta_{1}, \theta_{2} \in \mathbb{R}$ with $0<r_{1}<r_{2}$ and $0<\theta_{1}<\theta_{2}<\pi$. Choose $V=V(r, \theta)$, $W=W(r, \theta)$ to be smooth functions of their arguments such that (a) $V=1$ and $W=0$ whenever $r \notin\left(r_{1}, r_{2}\right)$ or $\theta \notin\left(\theta_{1}, \theta_{2}\right)$, (b) $V\left(r_{0}, \theta_{0}\right)<0$ for some $r_{0} \in\left(r_{1}, r_{2}\right)$ and $\theta_{0} \in\left(\theta_{1}, \theta_{2}\right)$, and (c) $W^{2}>-V r^{2} \sin ^{2} \theta$ everywhere. Clearly, the orbit of the asymptotically stationary Killing field $\partial / \partial t$ at $\left(r_{0}, \theta_{0}\right)$ is spacelike. However, no black hole or white hole is present in this spacetime, since at every point there exists some linear combination of the Killing fields $\partial / \partial t$ and $\partial / \partial \varphi$ which is timelike. (As already noted above, if a black hole were present, its event horizon would be null and every Killing field would have to be tangent to it. Hence, no Killing field could be timelike on the horizon.) Spacetimes with this character (i.e., possessing an "ergoregion" but no black or white hole) which are solutions to the Einstein-perfect-fluid equations have been numerically constructed by Butterworth and Ipser [7]. Theorem 4.1] is applicable to such spacetimes.

Another example of a spacetime containing an ergoregion for which our result for case (1) is applicable is provided in Figure 1.1. For the slice $\Sigma$ shown there, there is both a black hole (block $F$ ) and a white hole (block $A$ ), so this spacetime is not of class (1). However, the union of the blocks $B, D$, and $E$ - viewed as a spacetime in its own right - is a spacetime of class (1) with respect to $\Sigma$, despite the fact that the Killing orbits are spacelike in block $D$. Thus, Theorem 4.1 guarantees existence of a maximal slice extending from $M_{1}$ to $M_{2}$. On the other hand, it is worth noting that for the slice $\Sigma^{\prime}$ of Figure 1.1, the white hole region is block $A$, and the black hole region includes block $D$. Thus, no subset of this spacetime is of class (1) for the slice $\Sigma^{\prime}$ (or for any other slice spanning the asymptotic regions $M_{1}$ and $M_{3}$ ). Thus, Theorem 4.1 cannot be invoked to infer existence of a maximal slice extending from $M_{1}$ to $M_{3}$.

Examples of spacetimes of class (2) include the standard "non-extremal" stationary black hole solutions, such as the charged Kerr solutions with $e^{2}+a^{2}<m^{2}$ or the recently discovered "colored black hole" solutions to the Einstein-Yang-Mills equations. (Here $\Sigma$ can be taken to be any Cauchy surface for the domain of outer communications of one asymptotically flat region.) Note that the charged Kerr solutions with $a \neq 0$ contain an ergoregion exterior to the black and white holes. Note also that in the above examples, the intersection surface, $S$, of the black and white hole horizons has the topology of a sphere. However, using the methods of [14] one can construct asymptotically stationary spacetimes (not necessarily satisfying any 
Figure 1.1: The Penrose diagram of a spacetime which illustrates some applications of our theorems. Each point on this Figure represents a two sphere. The arrows represent the direction and character (spacelike or timelike) of Killing orbits. A spherically symmetric, asymptotically stationary spacetime, not necessarily satisfying any reasonable field equations, with the global structure displayed in this Figure can be easily constructed using the methods of 23. 
reasonable field equations or energy inequalities) such that $S$ has an arbitrary number of connected components, each of them having arbitrarily specifiable topology

Another example of a spacetime of class (2) is provided by Figure 1.1: The spacetime shown there is of class (2) with respect to the, hypersurface $\Sigma^{\prime \prime}$. Thus, Theorem 4.2 guarantees that there exists a maximal hypersurface with end $M_{1}$ and boundary $S$, which is asymptotically flat at $M_{1}$ and is asymptotically orthogonal to $X$.

We turn, now, to giving some examples of spacetimes for which our theorems do not apply. A simple class of examples of asymptotically flat, spacetimes with complete Killing field, $X$, timelike at each end, which contain neither a black hole nor white hole but fail to be of class (1) can be constructed by starting with any spacetime of class (1) and removing a Killing orbit which passes through $\Sigma$. (The compactness requirement on the "interior portion" of $\Sigma$ then will not be satisfied.) Inextendible spacetimes with similar properties also may easily be constructed. The Schwarzschild solution with negative mass provides a good example of such a spacetime.

A spacetime containing a black hole with respect to one or more asymptotic regions can fail to be in class (2) for a variety of reasons. The following are some examples of spacetimes which fail to be in class (2) for any choice of $\Sigma$ : (i) Any spacetime which contains a black hole with respect to one or more asymptotic regions, but not a white hole. The extended Schwarzschild spacetime with its white hole region removed provides a simple example of such a spacetime. (ii) Any spacetime which contains a black hole and white hole with respect to one or more ends, but the black and white hole horizons do not intersect. The "extreme" charged Kerr solutions (satisfying $e^{2}+a^{2}=m^{2}$ ) and the Majumdar-Papapetrou black holes (cf. e.g. [16]) provide examples of such spacetimes. Additional examples can be constructed in which the black hole horizon is a Killing horizon with non-constant surface gravity. In that case, Rácz and Wald [19] have recently shown that some of the generators of the horizon terminate in a parallelly propagated curvature singularity, and thus cannot intersect the generators of the horizon of any white hole that may be present. (iii) Numerous further examples can be constructed by starting with a spacetime of class (2) and removing suitable Killing orbits.

Although the above examples make clear that the assumptions made in case (2) are rather restrictive from the mathematical point of view, the following considerations indicate that these assumptions may not be very restrictive from the physical point of view. First, if Einstein's equation holds with matter satisfying suitable hyperbolic equations and energy conditions, and if both the matter fields and the spacetime are analytic, then Hawking has argued (cf. Propositions 9.3.5 and 9.3.6 of [18]) that each connected component of the event horizon of a black hole must be a Killing horizon. (This implies that either the asymptotically stationary Killing field must be normal to the horizon, or there must exist an additional Killing field in the spacetime.) Furthermore, if Einstein's equation with matter satisfying the dominant energy condition holds, then the surface gravity, $\kappa$, must be constant over each connected component of the horizon [4]: However, Rácz and Wald [19] have recently shown that if a spacetime possesses a one-parameter group of isometries with a Killing horizon such that $\kappa$ is constant and $\kappa \neq 0$, then a neighborhood of the horizon can be smoothly extended (if necessary) so that the Killing horizon is a bifurcate horizon. Thus, these combined results lend plausiblity to

\footnotetext{
${ }^{3}$ The construction of "toroidal black holes" given on p. 682 of [14] is easily generalized to other topologies. By construction the Killing orbits in "opposite" ends have opposite time orientation, and the existence of black hole regions follows from Lemma 3.2 proved below.
} 
the idea that the only physically relevant stationary black holes are ones whose event horizon has $\kappa=0$ (i.e., the "extremal case") and ones whose event horizon comprises a portion of a bifurcate Killing horizon. In the latter case, a white hole must also be present, and, under additional, presumably mild, assumptions, the spacetime should be of class (2).

In section 2 we define precisely the class of spacetimes we consider, and give some preliminary results. Some theorems concerning the structure of these spacetimes are presented in section 3; in particular, relationships are obtained between "having no black holes and no white holes" and compactness of domains of dependence of compact sets. Finally, section 4 contains our theorems on the existence of maximal hypersurfaces. In Appendix $\mathrm{A}$ we point out the existence of a generalization of our results to space-times which are "stationary-rotating". In Appendix B we give an alternative proof of one of our theorems under the additional hypothesis that the "timelike convergence condition" holds.

Acknowledgements: P.T.C. acknowledges useful discussions with R. Bartnik. Most of the work on this paper was done when P.T.C. was visiting the Centre for Mathematics and its Applications of the Australian National University, Canberra; he wishes to thank N. Trudinger and the CMA for friendly hospitality.

\section{Preliminary Definitions and Results on Asymptotically Sta- tionary Spacetimes}

Throughout this paper, we will assume that all manifolds are smooth, connected, Hausdorff, and paracompact, and all spacetimes are time oriented. For simplicity, unless otherwise specified, only smooth metrics will be considered, although all the results presented below would hold under appropropriate finite differentiability conditions. Unless specified otherwise, our notation and conventions will follow [22], with one exception: We shall define the domain of dependence $\mathcal{D}(\Sigma)$ of an achronal set $\Sigma$ as the set of all points $p$ such that every inextendible timelike (as opposed to causal) curve through $p$ intersects $\Sigma$. (This agrees with the definition given in 18] and corresponds when $\Sigma$ is closed to the closure of the domain of dependence as defined in 222.) We define $\stackrel{o}{\mathcal{D}}(\Sigma)=\operatorname{int} \mathcal{D}(\Sigma)$, where $\operatorname{int} \Omega$ stands for the interior of a set $\Omega$.

We begin by defining the notion of asymptotic stationarity. First, recall that a hypersurface is an embedded submanifold (without boundary) of codimension 1; a hypersurface with boundary is an embedded submanifold of codimension 1 with boundary; a slice is a closed, embedded submanifold without boundary.

Definition 2.1 A spacetime $\left(M, g_{a b}\right)$ possessing an acausal hypersurface $\Sigma$ (possibly with boundary) expressed in the form of a disjoint union $\Sigma=\Sigma_{1} \cup \Sigma^{\prime}$ will be called $(k, \alpha)$ asymptotically stationary with respect to the "end" $\Sigma_{1}$ if the following three conditions hold:

1. $\Sigma_{1}$ is diffeomorphic to $\mathbb{R}^{3} \backslash B\left(R_{1}\right)$, where $B\left(R_{1}\right)$ is a closed ball of radius $R_{1}$. There exists a coordinate system defined on some neighbourhood $\mathcal{O}_{1}$ of $\Sigma_{1}$ in which the coordinate components, $g_{\mu \nu}$, of the spacetime metric satisfy, for some $0<\alpha \leq 1,0<\lambda \leq 1, k \geq 2$,

$$
g_{00}<-C^{-1}, \quad\left|g_{\mu \nu}-\eta_{\mu \nu}\right| \leq C r^{-\alpha}
$$

\footnotetext{
${ }^{4}$ In particular, the indices $a, b, \ldots$ etc. are abstract tensor indices, while latin indices $i, j, \ldots$ etc. are component indices and run from 1 to 3 , and greek indices are component indices and run from 0 to 3 .
} 


$$
\begin{aligned}
& \forall Z^{i} \in \mathbb{R}^{3} \quad g_{i j} Z^{i} Z^{j} \geq C^{-1} \sum_{i=1}^{3}\left(Z^{i}\right)^{2} \\
& \mid \begin{array}{l}
\partial_{\sigma_{1}} \ldots \partial_{\sigma_{i}} g_{\mu \nu} \mid \leq C r^{-\alpha-i} \quad 1 \leq i \leq k \\
\left|\left(\partial_{\sigma_{1}} \ldots \partial_{\sigma_{k}} g_{\mu \nu}\right)(t, x)-\left(\partial_{\sigma_{1}} \ldots \partial_{\sigma_{k}} g_{\mu \nu}\right)(\tau, y)\right| \\
\leq C r^{-\alpha-k-\lambda}\left(|t-\tau|^{\lambda}+|x-y|^{\lambda}\right)
\end{array}
\end{aligned}
$$

for all $(t, x),(\tau, y) \in \mathcal{O}_{1}$ such that $|t-\tau| \leq 1,|x-y| \leq r(x) / 2$, and for some constant C. (Here $r \equiv\left(\sum_{i=1}^{3} x_{i}{ }^{2}\right)^{\frac{1}{2}}$.)

2. $\partial \bar{\Sigma}_{1}$, where $\bar{\Sigma}_{1}$ denotes the closure of $\Sigma_{1}$ considered as a subset of $\mathbb{R}^{3}$, is identified with a connected component of $\partial \Sigma^{\prime}$ by a diffeomorphism.

3. On $M$, there exists a Killing vector field $X$, the orbits of which are complete and which is uniformly timelike on $\Sigma_{1}\left(g_{a b} X^{a} X^{b}<-C^{-1}\right)$. Furthermore, in the coordinate system of point 1 we have as $r \rightarrow \infty$,

$$
X=\left(A+O\left(r^{-\alpha}\right)\right) \frac{\partial}{\partial t}+O\left(r^{-\alpha}\right) \frac{\partial}{\partial x^{i}}
$$

for some constant $A(\neq 0)$.

\section{Remarks:}

1. It is not too difficult to show from (2.1)-2.4) and from

$$
\nabla_{\mu} \nabla_{\nu} X_{\alpha}=R_{\lambda \mu \nu \alpha} X^{\lambda}
$$

(this last equation being a well known consequence of the Killing equations) that there exists constants $A \neq 0, B^{i}$ such that any Killing vector which is uniformly timelike for $r \geq R_{2}$, for some $R_{2} \geq R_{1}$, necessarily satisfies, in the coordinate system of definition (2.1),

$$
\begin{array}{ll} 
& X-A \frac{\partial}{\partial t}-B^{i} \frac{\partial}{\partial x^{i}}=O\left(r^{-\alpha}\right), \\
1 \leq i \leq k \quad & \partial_{\sigma_{1}} \ldots \partial_{\sigma_{i}} X^{\mu}=O\left(r^{-\alpha-i}\right),
\end{array}
$$

with an obvious uniform weighted Hölder condition satisfied by $\partial_{\sigma_{1}} \ldots \partial_{\sigma_{k}} X^{\mu}$. If $|B|<$ $|A|$ as must occur since $X$ is uniformly timelike on $\Sigma$, the constants $B^{i}$ can then be set to zero by constructing a coordinate system similar to that of Proposition 2.1 below, and subsequently performing an appropriate Lorentz transformation ("boost"). Thus, eq. (2.5) involves no loss of generality if $X$ is uniformly timelike 0 on $\Sigma_{1}$.

2. If (2.1) and (2.3) hold with $1 \leq i \leq k+1$, then (2.4) will hold as well (with any $0 \leq \lambda \leq 1$ ).

\footnotetext{
${ }^{5}$ If $X$ is merely timelike on $\Sigma_{1}$, then $X$ could asymptotically approach a null vector (so that $|B|=|A|$ ), in which case it could not be put in the form eq. (2.5). An example of such a spacetime is constructed in the Remark following the proof of Proposition A.1, Appendix A. We do not expect there to exist any metrics with a timelike Killing vector asymptotically approaching a null vector, and satisfying some reasonable field equations and/or energy inequalities; however, it might be of some interest to analyze this question for vacuum spacetimes.
} 
3. A generalization of this definition to asymptotically "stationary-rotating" spacetimes is given in Appendix A.

Let $\left(M, g_{a b}\right)$ with $\Sigma=\Sigma_{1} \cup \Sigma^{\prime}$ be asymptotically stationary with respect to $\Sigma_{1}$. We define $M_{1}$ by

$$
M_{1}=\bigcup_{t \in(-\infty, \infty)} \phi[X]_{t}\left(\Sigma_{1}\right)
$$

where throughout this paper $\phi[W]_{t}$ denotes the one-parameter group of isometries generated by a Killing vector field $W$. The following proposition, which gives some insight into the structure of asymptotically stationary spacetimes, will be used throughout (the coordinates the existence of which is claimed below will be referred to as Killing coordinates based on $\left.\Sigma \cap M_{1}\right)$ :

Proposition 2.1 (Killing time based on $\left.\Sigma \cap M_{1}\right)$ Let $\left(M, g_{a b}\right)$ with $\Sigma=\Sigma_{1} \cup \Sigma^{\prime}$ be asymptotically stationary with respect to $\Sigma_{1}$. Then there exists a global coordinate system on $M_{1}$ such that $M_{1} \approx \mathbb{R} \times\left(\mathbb{R}^{3} \backslash B\left(R_{1}\right)\right)$ with $\Sigma_{1}=\left\{x^{0}=0\right\}$, and

$$
\begin{gathered}
g_{00}<-C^{-1}, \quad \frac{\partial g_{\mu \nu}}{\partial t}=0 \\
\forall Z^{i} \in \mathbb{R}^{3} \quad g_{i j} Z^{i} Z^{j} \geq C^{-1} \sum_{i=1}^{3}\left(Z^{i}\right)^{2} \\
\left|\partial_{\sigma_{1}} \ldots \partial_{\sigma_{i}}\left(g_{\mu \nu}-\eta_{\mu \nu}\right)\right| \leq C r^{-\alpha-i} \quad 0 \leq i \leq k
\end{gathered}
$$

for some constant $C$; moreover, $\partial_{\sigma_{1}} \ldots \partial_{\sigma_{k}} g_{\mu \nu}$ satisfy an obvious weighted Hölder condition.

A generalization of Proposition 2.1 (Proposition A.1) is stated and proven in Appendix A. It should be noted that, as shown in the proof of Proposition A.1 given in Appendix A, one does not lose differentiability and/or uniform decay bounds of the derivatives of the metric when going to the Killing coordinates.

We now define the two classes of spacetimes which will be analyzed in this paper. These two classes correspond roughly to the two classes of Einstein-Yang-Mills solutions considered in [21].

Definition 2.2 (Spacetimes of class (a)) $\left(M, g_{a b}\right)$ is a strongly causal spacetime which contains a connected, acausal slice $\Sigma$ expressible as a disjoint union in the form $\Sigma=\bigcup_{i=1}^{I} \Sigma_{i} \cup \Sigma_{\text {int }}$, where $\Sigma_{\text {int }}$ is compact (with boundary). On $M$ there exists a Killing vector field $X$ such that $\left(M, g_{a b}\right)$ is $(k, \alpha)$-asymptotically stationary with respect to each $\Sigma_{i}$.

Let us emphasize that the hypersurface $\Sigma$ above is closed and has no boundary (as opposed to the case (b) defined below in which $\Sigma$ has a boundary). It should be stressed that we assume that $X$ approaches (a multiple of) $\partial / \partial t$ in all the asymptotic ends $\Sigma_{i}$. Thus, when more then one Killing vector and more than one end are present, we do not allow for situations in which one combination of Killing vectors is asymptotic to $\partial / \partial t$ in one end, and a different combination of Killing vectors has this property in another end. Note also that, under the conditions of Definition 2.2, $\Sigma$ is necessarily a complete Riemannian manifold with respect to the metric induced from $\left(M, g_{a b}\right)$. 
Definition 2.3 (Spacetimes of class (b)) $\left(M, g_{a b}\right)$ is a strongly causal spacetime which contains a connected, closed, acausal hypersurface $\Sigma$ with boundary $S$, such that $\Sigma$ is expressible as a disjoint union in the form $\Sigma=\bigcup_{i=1}^{I} \Sigma_{i} \cup \Sigma_{\text {int }}$, where $\Sigma_{\text {int }}$ is compact (with boundary). On $M$ there exists a Killing vector field $X$ such that $\left(M, g_{a b}\right)$ is $(k, \alpha)$-asymptotically stationary with respect to each $\Sigma_{i}$, and $X$ is tangent to $S$ (so that the isometries, $\phi[X]_{t}$, map $S$ into $S$ ).

Note that we do not assume that $S$ is connected. Note also that under the conditions of Definition $2.3 \Sigma$ is necessarily a complete Riemannian manifold with boundary $S$.

By a standard theorem ( $c f .$, e.g., [18, 22]), the future Cauchy horizon, $\mathrm{H}^{+}$, of any closed, achronal set is generated by null geodesics which either are past inextendible or have past endpoint on the edge of that set. For spacetimes of class (a), $\Sigma$ is edgeless. However, for spacetimes of class (b) the edge of $\Sigma$ is $S$. For spacetimes of class (b), we define $\mathcal{H}_{+}$to consist of the portion of $\mathrm{H}^{+}$which is generated by null geodesics with past endpoint on $S$. Similarly, we define $\mathcal{H}_{-}$to consist of the portion of the of the past Cauchy horizon, $H^{-}$, of $\Sigma$ generated by null geodesics with future endpoint on $S$. Since $I^{+}(S)$ and $I^{-}(S)$ do not lie in $\mathcal{D}(\Sigma)$ and since the boundary, $\partial \mathcal{D}(\Sigma)$, of $\mathcal{D}(\Sigma)$ is the entire Cauchy horizon, $\partial \mathcal{D}(\Sigma)=H=H^{+} \cup H^{-}$, $(c f .$, e.g., [18, 22]), it follows that

$$
\mathcal{H}_{+}=J^{+}(S) \cap \mathcal{D}(\Sigma), \quad \mathcal{H}_{-}=J^{-}(S) \cap \mathcal{D}(\Sigma)
$$

We define

$$
\mathcal{H}=\mathcal{H}_{+} \cup \mathcal{H}_{-} .
$$

In our analysis of spacetimes of class (b) given in the next section, we will focus our attention on the manifold with boundary,

$$
M^{\prime}=\stackrel{o}{\mathcal{D}}(\Sigma) \cup \mathcal{H}
$$

It should be stressed that $\mathcal{H}$ may comprise only a portion of the boundary of $\mathcal{D}(\Sigma)$, as illustrated in Figure 2.1.

For spacetimes of class (a) and (b), we define

$$
M_{\mathrm{ext}}=\bigcup_{i=1}^{I} M_{i},
$$

where $M_{i}=\bigcup_{t \in \mathbb{R}} \phi[X]_{t}\left(\Sigma_{i}\right)$. and we set

$$
M_{\mathrm{int}}=M \backslash M_{\mathrm{ext}} \text {. }
$$

Note that by construction we have $\phi[X]_{t}\left[M_{\text {ext }}\right]=M_{\text {ext }}$ for all $t$, whence $\phi[X]_{t}\left[M_{\text {int }}\right]=M_{\text {int }}$.

In both cases (a) and (b), for an asymptotic end $M_{i}$ we define "the black hole region with respect to $M_{i} "$ by

$$
\mathcal{B}_{i}=M \backslash I^{-}\left(M_{i}\right),
$$

with the "white hole region with respect to $M_{i}$ " defined dually,

$$
\mathcal{W}_{i}=M \backslash I^{+}\left(M_{i}\right) .
$$


Figure 2.1: A Penrose diagram of a spacetime in which $\mathcal{H}$ is not the entire Cauchy horizon of $\Sigma$. In this spacetime $\partial \mathcal{D}(\Sigma)$ has two connected components, $\mathcal{H}=\mathcal{H}_{+} \cup \mathcal{H}_{-}$and $\mathcal{H}^{\prime}$.

It is clear that (2.14)-(2.15) are equivalent to the standard definition 6 using $\mathcal{I}$ [22] [18], whenever $\mathcal{I}$, understood as a conformal completion based on a Bondi coordinate system, exists $\mathbb{J}$.

We define the black hole region, $\mathcal{B}$, of the spacetime by,

$$
\mathcal{B}=M \backslash I^{-}\left(M_{\text {ext }}\right)=\bigcap_{i=1}^{I} \mathcal{B}_{i},
$$

and similarly define the white hole region by,

$$
\mathcal{W}=M \backslash I^{+}\left(M_{\text {ext }}\right)=\bigcap_{i=1}^{I} \mathcal{W}_{i} .
$$

As already mentioned in the Introduction, it should be emphasized that it is possible for spacetimes of class (a) to have $\mathcal{B}_{i} \neq \emptyset$ for some $i$ and yet have $\mathcal{B}=\emptyset$. The union of blocks $B$, $D$, and $E$ of Figure 1.1 provides an illustration of such a spacetime. On the other hand, in case (b), since $\phi[X]_{t}[S]=S$, it follows from Corollary 3.2, proven in the next section, that $\mathcal{B}, \mathcal{W} \neq \emptyset$, and, indeed, $J^{+}(S) \subset \mathcal{B}, J^{-}(S) \subset \mathcal{W}$. Finally, it should be mentioned that Proposition 3.3

\footnotetext{
${ }^{6}$ It is customary to define the black hole, respectively the white hole, with respect to the asymptotic end $M_{i}$ as $M \backslash J^{-}\left(\mathcal{I}^{+} ; \tilde{M}\right)$, respectively $M \backslash J^{+}\left(\mathcal{I}^{-} ; \tilde{M}\right)$, where $J^{ \pm}(\Omega ; \tilde{M})$ denotes the causal past and future of the set $\Omega$ in the conformally extended manifold $\tilde{M}$.

${ }^{7}$ More generally, the equivalence of 2.14 2.15) with the standard definition can be established whenever the manifold $M_{i}$ admits a $\mathcal{I}$ satisfying the conditions of [15], and the Ricci tensor falls off fast enough (in the sense of the note added in proof (3) of [1] ) in the asymptotic end in question. The advantage of (2.14)-(2.15) is, that one avoids all the issues related to the possible low differentiability and/or incompleteness of $\mathcal{I}$. On the other hand the definition given here does not generalize in any obvious way to non-stationary space-times. It should be mentioned that if $M_{i}$ is assumed to be vacuum, then a smooth $\mathcal{I}$ satisfying the requirements of 15 necessarily exists ( $c f$. [11] [Appendix], and also [6, 20]).
} 
below shows that $\mathcal{B}_{i}$ and $\mathcal{W}_{i}$ are independent of that arbitrariness in the definition of $M_{\text {ext }}$, which is related to the arbitrariness of separation of $\Sigma$ into $\Sigma_{\text {ext }}$ and $\Sigma_{\text {int }}$.

Since $M_{\text {ext }}$ is $\phi[X]_{s}$ invariant, so are $I^{ \pm}\left(M_{\text {ext }}\right)$, we thus have

$$
\forall t \in \mathbb{R} \quad \phi[X]_{t}(\mathcal{B})=\mathcal{B}, \quad \phi[X]_{t}(\mathcal{W})=\mathcal{W} .
$$

It follows immediately that the boundaries of $\mathcal{B}$ and $\mathcal{W}$ - known as the event horizons of $\mathcal{B}$ and $\mathcal{W}$, respectively, are null surfaces which are invariant under $\phi[X]_{t}$. As already mentioned in the Introduction, this implies that if the Killing field $X$ is strictly timelike in $M$, then $\mathcal{B}=\mathcal{W}=\emptyset$. Furthermore, as shown explicitly in the Introduction, in case (a), it is possible to have $\mathcal{B}=\mathcal{W}=\emptyset$ even though $X$ becomes spacelike in certain regions (called ergoregions) of $M$. Similarly, as explicitly demonstrated by the example of the Kerr metric, in case (b), $X$ may be spacelike within $\stackrel{o}{\mathcal{D}}(\Sigma)$ and yet have $\stackrel{o}{\mathcal{D}}(\Sigma) \cap \mathcal{B}=\emptyset, \stackrel{o}{\mathcal{D}}(\Sigma) \cap \mathcal{W}=\emptyset$.

In this paper, we shall be concerned with the issue of the existence of asymptotically flat, maximal hypersurfaces and foliations by maximal hypersurfaces in spacetimes of class (a) and (b). The notion of asymptotic flatness of a hypersurface is defined as follows:

Definition 2.4 Let $\left(M, g_{a b}\right)$ be a space-time in which (2.1)-(2.4) hold. A hypersurface $\Sigma \subset M$ will be called asymptotically flat if $\Sigma \cap \mathcal{O}_{1}\left(\mathcal{O}_{1}\right.$ - as in point 11 of Definition 2.1) is a graph $t=u(x)$ of a function $u$ satisfying

$$
|u|+r\left|\partial_{i} u\right|+\ldots+r^{k+1}\left|\partial_{i_{1}} \ldots \partial_{i_{k+1}} u\right| \leq \begin{cases}C(1+r)^{1-\alpha}, & 0<\alpha<1 \\ C(1+\ln (1+r)), & \alpha=1\end{cases}
$$

(together with an appropriate weighted Hölder condition on $\partial_{i_{1}} \ldots \partial_{i_{k+1}} u$ ).

By a common abuse of terminology, we shall say that a spacelike hypersurface $\Sigma$ is maximal if the trace of the extrinsic curvature of $\Sigma$, perhaps defined distributionally, vanishes. It follows that a maximal hypersurface is locally maximal in a variational sense, cf. e.g. 3].

Let $t$ be a time function on $M$, i.e., a continuous function strictly increasing along any future directed causal curve. We shall use the notation

$$
\Sigma_{s}(t)=\{p \in M: t(p)=s\}
$$

for the level sets of $t$. The sets $\Sigma_{s}(t)$ are closed, and if $t \in C^{k}(M), k \geq 1$, with $d t$ non-vanishing on an open subset $\mathcal{O}$ of $M$, then $\Sigma_{s}(t) \cap \mathcal{O}$ are $C^{k}$ acausal submanifolds of $M$.

Let $\Sigma_{t}, t \in I$, where $I \subset \mathbb{R}$, be a family of hypersurfaces. We shall say that $\Sigma_{t}$ foliates $\mathcal{O}$ if there exists a function $t$ on $\mathcal{O}$ such that $\Sigma_{s}=\Sigma_{s}(t)$. Note that this implies in particular that $\mathcal{O}=\bigcup_{t \in I} \Sigma_{t}$, and that the implication $\left(t \neq t^{\prime}\right) \Longrightarrow\left(\Sigma_{t} \cap \Sigma_{t^{\prime}}=\emptyset\right)$ holds. If $\Sigma_{t}$ foliate $\mathcal{O}$, then the family $\Sigma_{t}$ will be called a foliation (of $\mathcal{O}$ ). The function $t$ will be said associated to the foliation $\Sigma_{t}$. If $d t$ is nowhere vanishing on $\mathcal{O}$ and $I$ is connected, with $0 \in I$, then $t$ induces a natural diffeomorphism $\varphi: \mathcal{O} \longrightarrow I \times \Sigma_{0}$ by setting $\varphi(p)=(t(p), q(p))$, where $q(p)$ is the intersection of $\Sigma_{0}$ with the integral curve of $\nabla t$ through $p$.

We conclude this section by introducing some terminology to be used in the next section ( $c f$. [17] [Chapter VII]). 
Definition 2.5 Let $M$ be a manifold, let $\psi_{t}: M \rightarrow M$ denote a one-parameter group of diffeomorphisms and let $\gamma_{p}$ denote the orbit of $\psi_{t}$ through $p \in M$. The $\alpha$-limit set $\alpha_{p}$, of $\gamma_{p}$ is defined as the set of all $q \in M$ such that $q$ can be expressed as $q=\lim _{i \rightarrow \infty} \psi_{t_{i}}(p)$ for some $t_{i} \rightarrow-\infty$. The $\omega$-limit set, $\omega_{\sigma}$, of $\gamma_{p}$ is defined similarly, except that $\left\{t_{i}\right\}$ is required to diverge to $+\infty$.

\section{Causal Properties of Spacetimes of Classes (a) and (b)}

In this section we establish some properties of the spacetimes introduced in the previous section. Since our results and proofs for case (b) are closely analogous to those of case (a), in general, we will merely state the results for case (b) without proof, or will simply indicate the modifications to the proof for case (a).

Our first result is the following:

Proposition 3.1 Let $\left(M, g_{a b}\right)$ be a spacetime of class (a), with slice $\Sigma$. Then $\mathcal{D}(\Sigma)$ contains complete Killing orbits, i.e., if $p \in \mathcal{D}(\Sigma)$ then $\phi[X]_{t}(p) \in \mathcal{D}(\Sigma)$ for all $t \in \mathbb{R}$. Moreover $\phi[X]_{t}(\Sigma)$ are Cauchy surfaces for $\stackrel{\circ}{\mathcal{D}}(\Sigma)$, for all $t \in \mathbb{R}$.

Proof: We wish to show that $\phi[X]_{t}[\mathcal{D}(\Sigma)]=\mathcal{D}(\Sigma)$ for all $t \in \mathbb{R}$. Let

$$
\Sigma_{t}=\phi[X]_{t}[\Sigma]
$$

Since the $\phi[X]_{t}$ 's are isometries we have $\phi[X]_{t}[\mathcal{D}(\Sigma)]=\mathcal{D}\left(\Sigma_{t}\right)$, and the proposition will be proven by showing that $\mathcal{D}\left(\Sigma_{t}\right)=\mathcal{D}(\Sigma)$.

Let $\gamma \subset \stackrel{o}{\mathcal{D}}(\Sigma)$ be an inextendible (in $\stackrel{o}{\mathcal{D}}(\Sigma)$ ), piecewise differentiable timelike curve, and define

$$
I=\left\{t \in \mathbb{R}: \phi[X]_{t}(\Sigma) \cap \gamma \neq \emptyset\right\} .
$$

Since $\gamma$ is a timelike curve and all the $\phi[X]_{t}(\Sigma)$ 's are spacelike it follows that $I$ is open. From the fact that $\Sigma$ is a Cauchy surface for $\stackrel{o}{\mathcal{D}}(\Sigma)$ we have $0 \in I$, thus $I \neq \emptyset$. Let $t_{i} \in I$ be such that $t_{i} \rightarrow t \in \mathbb{R}$, let $q_{i}=\phi[X]_{-t_{i}}\left(\gamma \cap \phi[X]_{t_{i}}(\Sigma)\right) \in \Sigma$. Asymptotic flatness (cf. Proposition 2.1) implies that $q_{i} \in K$ for some compact subset of $\Sigma$. Passing to a subsequence, still denoted by $t_{i}$, there exists $q \in K$ such that $q_{i} \rightarrow q$. Continuity of $\phi[X]_{s}(p)$ with respect to $s$ and $p$ imply that the sequence $\phi[X]_{t_{i}}\left(q_{i}\right)=\gamma \cap \phi[X]_{t_{i}}(\Sigma)$ accumulates at $\phi[X]_{t}(q)$, thus $\gamma$ accumulates at $\phi[X]_{t}(q)$ and inextendability of $\gamma$ implies that $t \in I$. Thus $I=\mathbb{R}$ and the result follows.

By similar arguments, we obtain the following Proposition for case (b). (The only modifications needed to the proof concerns analysis of the possibility that the accumulation point $x$ might lie on $S$; in that case, the invariance of $S$ under the isometries should be used.)

Proposition 3.2 Let $\left(M, g_{a b}\right)$ be a spacetime of class (b). Then for $p \in \mathcal{D}(\Sigma)$, respectively $p \in \stackrel{o}{\mathcal{D}}(\Sigma), t \in \mathbb{R}$, we have $\phi[X]_{t}(p) \in \mathcal{D}(\Sigma)$, respectively $\phi[X]_{t}(p) \in \stackrel{o}{\mathcal{D}}(\Sigma)$. Moreover the hypersurfaces $\phi[X]_{t}(\Sigma \backslash S)$ are Cauchy surfaces for $\stackrel{o}{\mathcal{D}}(\Sigma)$, and $\mathcal{D}\left[\phi[X]_{t}(\Sigma)\right]=\mathcal{D}(\Sigma)$.

As a corollary of Propositions 3.1 and 3.2 we have the following: 
Corollary 3.1 Let $\left(M, g_{a b}\right)$ be a spacetime of class (a) or (b). Then $M \backslash \stackrel{o}{\mathcal{D}}(\Sigma) \subset \mathcal{B} \cup \mathcal{W}$. In particular, we have $M_{\text {ext }} \subset \stackrel{o}{\mathcal{D}}(\Sigma)$.

Proof: That $M_{\text {ext }} \subset \stackrel{o}{\mathcal{D}}(\Sigma)$ follows immediately from the definition of $M_{\text {ext }}$ and Propositions 3.1 and 3.2. Now, suppose that $p \notin \mathcal{B} \cup \mathcal{W}$ and $p \notin \stackrel{o}{\mathcal{D}}(\Sigma)$. Then there exist $q_{-}, q_{+} \in M_{\text {ext }}$ such that $p \in I^{-}\left(q_{+}\right) \cap I^{+}\left(q_{-}\right)$. Without loss of generality, we may assume that $q_{-} \in I^{-}[\Sigma]$ and $q_{+} \in I^{+}[\Sigma]$. Since $p \notin \stackrel{o}{\mathcal{D}}(\Sigma)$, there exists $p^{\prime} \in I^{-}\left(q_{+}\right) \cap I^{+}\left(q_{-}\right)$such that $p^{\prime} \notin \mathcal{D}(\Sigma)$. Let $\lambda^{\prime}{ }_{-}$be a future directed timelike curve which connects $q_{-}$to $p^{\prime}$, and let $\lambda^{\prime}{ }_{+}$be a past directed timelike curve which connects $q_{+}$to $p^{\prime}$. Since $\Sigma$ is achronal, $\lambda^{\prime}{ }_{-}$and $\lambda^{\prime}{ }_{+}$cannot both intersect $\Sigma$. Suppose that $\lambda^{\prime}$ - fails to intersect $\Sigma$. Then since $p^{\prime} \notin \mathcal{D}(\Sigma)$, there exists a future inextendible timelike curve starting at $p^{\prime}$ which fails to intersect $\Sigma$. By adjoining this curve to $\lambda^{\prime}{ }_{-}$, we obtain a future inextendible timelike curve from $q_{-} \in I^{-}[\Sigma]$ which fails to intersect $\Sigma$. This contradicts the fact that $q_{-} \in M_{\text {ext }} \subset \stackrel{o}{\mathcal{D}}(\Sigma)$. A similar argument yields a contradiction if $\lambda^{\prime}+$ fails to intersect $\Sigma$.

For some arguments it will be convenient to have global hyperbolicity of the objects at hand (but it should be stressed that we are not assuming global hyperbolicity unless indicated otherwise). The main significance of Proposition 3.1 is that it will have the effect of allowing us to restrict consideration in case (a) to globally hyperbolic spacetimes for which the hypersurface $\Sigma$ is a Cauchy surface. Namely, in case $(\mathrm{a})$, since $\mathcal{D}(\Sigma)$ is invariant under the isometries $\phi[X]_{t}$, so is $M^{\prime}=\stackrel{o}{\mathcal{D}}(\Sigma)$. Hence, the spacetime $\left(M^{\prime}, g_{a b}\right)$ also is of class (a) (with the same asymptotic regions) but is globally hyperbolic with Cauchy surface $\Sigma$. Existence of a maximal hypersurface in $\left(M^{\prime}, g_{a b}\right)$ implies existence of a maximal hypersurface in $\left(M, g_{a b}\right)$ and existence of a maximal foliation of the spacetime $\left(M^{\prime}, g_{a b}\right)$ implies existence of a maximal foliation of a subset of $\left(M, g_{a b}\right)$ which covers the entire asymptotic region. Similarly, in case (b) Proposition 3.2 will have the effect of allowing us to restrict consideration to $M^{\prime}=\stackrel{o}{\mathcal{D}}(\Sigma) \cup \mathcal{H}$.

Most of the remainder of this section will be devoted to proving the equivalence of a "no black or white hole" condition to a compactness condition. In preparation for this, we prove the following lemmas:

Lemma 3.1 Let $\left(M, g_{a b}\right)$ be a spacetime of class (a) or (b) and let $Q \subset M$ be any isometry invariant subset, i.e., $\phi[X]_{t}[Q]=Q$. Let $M_{i}$ denote any "end" (cf. eq. (2.9)). Then either $I^{+}(Q) \supset M_{i}$ or $I^{+}(Q) \cap M_{i}=\emptyset$. Similarly either $I^{-}(Q) \supset M_{i}$ or $I^{-}(Q) \cap M_{i}=\emptyset$.

Proof: Suppose $I^{+}(Q) \cap M_{i} \neq \emptyset$. Let $x \in I^{+}(Q) \cap M_{i}$. Since $\phi[X]_{t}\left[I^{+}(Q)\right]=I^{+}\left[\phi[X]_{t}(Q)\right]=$ $I^{+}(Q)$ and $\phi[X]_{t}\left(M_{i}\right)=M_{i}$, it follows that $\phi[X]_{t}(x) \in I^{+}(Q) \cap M_{i}$ for all $t \in \mathbb{R}$, i.e., $\gamma_{x} \subset I^{+}(Q) \cap M_{i}$ where $\gamma_{x}$ denotes the orbit of $x$ under $\phi[X]_{t}$. However, from the asymptotic form of the metric given by Proposition 2.1, it follows that given any $x, y \in M_{i}$, there exists a sufficiently large negative $t$ such that $y \in I^{+}\left[\phi[X]_{t}(x)\right]$. This implies that $M_{i} \subset I^{+}\left(\gamma_{x}\right) \subset$ $I^{+}(Q)$.

We note the following Corollaries (cf. also [8] [Corollary, p. 136]):

Corollary 3.2 Let $\left(M, g_{a b}\right)$ be a space-time of class (a), let $Q$ be a compact set such that $\phi[X]_{t}(Q)=Q, t \in \mathbb{R}$. Then we have $\mathcal{B} \cup \mathcal{W} \neq \emptyset$. If moreover $Q \subset \stackrel{o}{\mathcal{D}}(\Sigma)$ then $\mathcal{B} \cap \mathcal{W}$ is nonempty, with $Q \subset \mathcal{B} \cap \mathcal{W}$. In particular if $p \in \stackrel{\circ}{\mathcal{D}}(\Sigma)$ and $\left.X\right|_{p}=0$, then $p \in \mathcal{B} \cap \mathcal{W}$. 


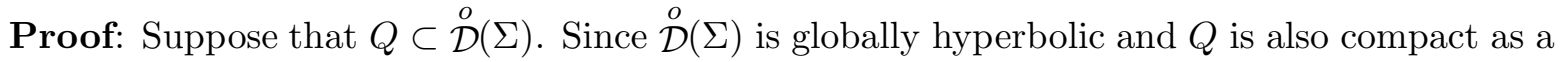
subset of $\stackrel{o}{\mathcal{D}}(\Sigma)$ the inclusions $M_{i} \subset I^{+}(Q), M_{i} \subset I^{-}(Q)$ are not possible (cf. e.g. [13]). Thus Lemma 3.1 gives $M_{i} \cap I^{+}(Q)=M_{i} \cap I^{-}(Q)=\emptyset, i=1, \ldots, I$, which implies $Q \subset \mathcal{B} \cap \mathcal{W}$. If on the other hand $Q \not \subset \stackrel{o}{\mathcal{D}}(\Sigma)$, then $M \backslash \stackrel{o}{\mathcal{D}}(\Sigma) \neq \emptyset$ and $\mathcal{B} \cup \mathcal{W} \neq \emptyset$ follows from Corollary 3.1.

Corollary 3.3 Let $\left(M, g_{a b}\right)$ be a spacetime of class (b). Then $S \subset \mathcal{B} \cap \mathcal{W}$.

Proof: We have $\phi[X]_{t}[S]=S$, but $I^{+}(S) \cap \Sigma_{\text {ext }}=I^{-}(S) \cap \Sigma_{\text {ext }}=\emptyset$ since $\Sigma$ is achronal.

Before stating our final corollary of lemma 3.1, we introduce the following terminology:

Definition 3.1 For $p \in \stackrel{o}{\mathcal{D}}(\Sigma)$ we shall say that an orbit $\gamma_{p}(t) \equiv \phi[X]_{t}(p)$ is future oriented, respectively past oriented, if there exists an increasing sequence $t_{i} \in \mathbb{R}, i \in \mathbf{Z}$, with $\left|t_{i}\right| \longrightarrow|i| \rightarrow \infty$ $\infty$ such that $\phi[X]_{t_{i+1}}(p) \in I^{+}\left(\phi[X]_{t_{i}}(p)\right)$, respectively $\phi[X]_{t_{i+1}}(p) \in I^{-}\left(\phi[X]_{t_{i}}(p)\right)$. An orbit will be said time oriented if it is future or past oriented.

If $X_{p}$ is timelike future pointing, respectively past pointing, then $\gamma_{p}(t)$ is clearly future oriented, respectively past oriented. It should be noted, that if there exists $p$ and $\Delta t \in \mathbb{R}$ such that $\phi[X]_{\Delta t}(p) \in I^{+}(p)$, then the orbit $\gamma_{p}$ of $\phi[X]$ through $p$ is necessarily time oriented, for then for any $q \in \gamma_{p}$ we have $\phi[X]_{\Delta t}(q) \in I^{+}(q)$, and the desired sequence is given by $i \Delta t, i \in \mathbf{Z}$.

Corollary 3.4 Let $\left(M, g_{a b}\right)$ be a spacetime of class (a) or (b) such the orbits of $X$ are future oriented at each "end", $M_{i}$. Let $p \notin \mathcal{W}$. Then, there exists $T \in \mathbb{R}$ such that for all $t>T$, we have $\phi[X]_{t}[p] \in I^{+}\left[\Sigma_{\text {ext }}\right]$. Similarly, if $p \notin \mathcal{B}$, then there exists $T^{\prime} \in \mathbb{R}$ such that for all $t<T^{\prime}$, we have $\phi[X]_{t}[p] \in I^{-}\left[\Sigma_{\text {ext }}\right]$. Furthermore, if $p \notin \mathcal{B} \cup \mathcal{W}$, then the set $\left\{t \in \mathbb{R}: \phi[X]_{t}(p) \in \Sigma\right\}$ is nonempty and compact.

Proof: If $p \notin \mathcal{W}$, then by lemma 3.1, the past of the Killing orbit through $p$ must contain at least one "end", $M_{i}$. Consequently, there exists $T \in \mathbb{R}$ such that $\phi[X]_{T}[p] \in I^{+}\left[\Sigma_{\text {ext }}\right]$. The first claim of the corollary then follows immediately by applying $\phi[X]_{t^{\prime}}$ to this relation, for any $t^{\prime}>0$, using the fact that for $t^{\prime}>0, \phi[X]_{t^{\prime}}$ takes $\Sigma_{\text {ext }}$ into $I^{+}\left[\Sigma_{\text {ext }}\right]$. Similar arguments establish the second claim. Finally, if $p \notin \mathcal{B} \cup \mathcal{W}$, then $\left\{t \in \mathbb{R}: \phi[X]_{t}(p) \in \Sigma\right\}$ is a closed subset of $\left[T^{\prime}, T\right]$, and hence is compact. To prove that this set is nonempty, we note that $\phi[X]_{t}$ enters both $I^{-}[\Sigma]$ and $I^{+}[\Sigma]$. Furthermore, by Corollary 3.1, $p \in \mathcal{D}(\Sigma)$, and by Propositions 3.1 and 3.2, $\phi[X]_{t} \subset \stackrel{o}{\mathcal{D}}(\Sigma)$. However, $\stackrel{o}{\mathcal{D}}(\Sigma)$ is globally hyperbolic, so it can be expressed as the disjoint union $\stackrel{o}{\mathcal{D}}(\Sigma)=\left[I^{-}[\Sigma] \cap \stackrel{o}{\mathcal{D}}(\Sigma)\right] \cup[\Sigma \cap \stackrel{o}{\mathcal{D}}(\Sigma)] \cup\left[I^{+}[\Sigma] \cap \stackrel{o}{\mathcal{D}}(\Sigma)\right]$. Hence, it follows that a continuous curve in $\stackrel{\circ}{\mathcal{D}}(\Sigma)$ which enters both $I^{-}[\Sigma]$ and $I^{-}[\Sigma]$ must intersect $\Sigma$.

The following Proposition gives an invariant characterization of $\mathcal{B}_{i}$ and $\mathcal{W}_{i}$ :

Proposition 3.3 Let $\mathcal{O}_{i}$ be the connected component of the set $\left\{p \in \stackrel{o}{\mathcal{D}}(\Sigma)\right.$ : the orbit $\gamma_{p}$ through $p$ is time oriented $\}$ which contains $M_{i}$. Then $\mathcal{B}_{i}=M \backslash I^{-}\left(\mathcal{O}_{i}\right), \mathcal{W}_{i}=M \backslash I^{+}\left(\mathcal{O}_{i}\right)$.

Proof: By definition $M_{i} \subset \mathcal{O}_{i}$, thus $M \backslash I^{-}\left(\mathcal{O}_{i}\right) \subset \mathcal{B}_{i}$. Now $\partial\left(M \backslash I^{-}\left(M_{i}\right)\right) \cap \mathcal{O}_{i}$ is an achronal subset of $\mathcal{O}_{i}$ which is invariant under $\phi[X]_{t}$ by construction. Because all the orbits of $X$ in $\mathcal{O}_{i}$ are time oriented, the only such subset of $\mathcal{O}_{i}$ is the empty set, and the reverse inclusion follows. 
Lemma 3.2 Let $\left(M, g_{a b}\right)$ be a spacetime of class (a) such that the orbits of $\phi[X]_{t}$ fail to have the same (past or future) orientation on all ends, i.e. suppose $A_{i} A_{j}<0$ for some $i$ and $j$, where $A_{i}$ is the constant $A$ of Definition 2.1 (cf. (2.5)) in the region $M_{i}$. Then $\mathcal{B} \cup \mathcal{W} \neq \emptyset$, where $\mathcal{B}$ and $\mathcal{W}$ were defined by eqs. 2.10) and (2.17). Moreover $I^{+}\left(M_{i}\right) \cap M_{j}=\emptyset, I^{-}\left(M_{i}\right) \cap M_{j}=\emptyset$.

Remark: Note that the last part of Lemma 3.2 says, that two ends with different Killingtime orientations cannot communicate with each other (in fact, the argument here shows the stronger statement that if the orbit $\gamma_{p}$ has time orientation which is opposite to that of the orbit $\gamma_{q}$, then $\left.I^{+}(p) \cap I^{-}(q)=I^{-}(p) \cap I^{+}(q)=\emptyset\right)$, while the first part says that, reversing time orientation if necessary, there exists a region which cannot send a signal to any of the ends $M_{i}$.

Proof: Let $M_{+}=\bigcup_{A_{i}>0} M_{i}$ and let $M_{-}=\bigcup_{A_{i}<0} M_{i}$. We show first that $I^{+}\left(M_{-}\right) \cap M_{+}=\emptyset$. Namely, suppose that $x \in M_{+}$and $y \in M_{-}$were such that $x \in I^{+}(y)$. By choosing $t$ sufficiently large negative, we can ensure that $\phi[X]_{t}(x) \in I^{-}[\Sigma], \phi[X]_{t}(y) \in I^{+}[\Sigma]$. However, by isometry invariance, we have $\phi[X]_{t}(x) \in I^{+}\left[\phi[X]_{t}(y)\right]$, which contradicts the achronality of $\Sigma$. Similarly, we have $I^{-}\left(M_{-}\right) \cap M_{+}=\emptyset$ and the same relations with $M_{+}$and $M_{-}$interchanged.

Now, $I^{+}\left(M_{+}\right)$and $I^{+}\left(M_{-}\right)$are open sets. If their union failed to cover $M$, then $\mathcal{W} \neq \emptyset$. If their union covers $M$, connectedness of $M$ implies that they cannot be disjoint. Let $x \in I^{+}\left(M_{+}\right) \cap$ $I^{+}\left(M_{-}\right)$. Then $I^{+}(x) \subset I^{+}\left(M_{+}\right) \cap I^{+}\left(M_{-}\right)$and hence $I^{+}(x) \cap M_{-}=\emptyset, I^{+}(x) \cap M_{+}=\emptyset$. Thus, $I^{+}(x) \cap M_{\text {ext }}=\emptyset$, i.e. $x \in \mathcal{B}$, so $\mathcal{B} \neq \emptyset$.

Similarly one proves:

Lemma 3.3 Let $\left(M, g_{a b}\right)$ be a spacetime of class (b) such that the orbits of $\phi[X]_{t}$ fail to have the same (past or future) orientation on all ends. Then

$$
(\mathcal{B} \cup \mathcal{W}) \cap \stackrel{o}{\mathcal{D}}(\Sigma) \neq \emptyset
$$

Lemma 3.4 Let $\left(M, g_{a b}\right)$ be a spacetime of class (a) or (b) with $A_{i}>0$ for all $i$. Let $p \in \stackrel{o}{\mathcal{D}}(\Sigma)$ and let $\omega_{p}$, respectively $\alpha_{p}$, denote the $\omega$-limit set, respectively the $\alpha$-limit set of the orbit $\phi[X]_{t}(p)$. Then $\omega_{p} \subset \mathcal{B}, \alpha_{p} \subset \mathcal{W}$.

Proof: By duality it is sufficient to prove $\omega_{p} \subset \mathcal{B}$. It follows immediately from definition 2.5 that $\phi[X]_{t}\left[\omega_{p}\right]=\omega_{p}$. Hence, by lemma 3.1 it suffices to show that $I^{+}\left(\omega_{p}\right) \not \supset M_{\text {ext }}$. However, since $A_{i}>0$, for all $t>0$ we have $I^{+}(p) \cap M_{\text {ext }} \supset \phi[X]_{t}\left[I^{+}(p) \cap M_{\text {ext }}\right]=I^{+}\left[\phi[X]_{t}(p)\right] \cap M_{\text {ext }}$, from which it follows that $I^{+}(p) \cap M_{\text {ext }} \supset I^{+}\left(\omega_{p}\right) \cap M_{\text {ext }}$. However, since $p \in \stackrel{o}{\mathcal{D}}(\Sigma), J^{+}(p)$ intersects $\Sigma$ compactly (cf. e.g. [13]), so there exists $y \in \Sigma_{\text {ext }} \subset M_{\text {ext }}$ such that $y \notin I^{+}(p)$. Thus, $I^{+}\left(\omega_{p}\right) \not \supset M_{\text {ext }}$, as we desired to show.

Finally, in the course of proving the second half of theorem 3.1, we shall appeal to the following lemma:

Lemma 3.5 Let $\left(M, g_{a b}\right)$ be a spacetime of class (a), and suppose that $\mathcal{D}\left(\Sigma_{\mathrm{int}}\right)$ is compact. Then $\mathcal{D}\left(\Sigma_{\text {int }}\right) \subset \stackrel{o}{\mathcal{D}}(\Sigma)$. 
Proof: Clearly, $\mathcal{D}\left(\Sigma_{\text {int }}\right) \subset \mathcal{D}(\Sigma)$, so we need only prove a contradiction with the possibility that there exists $p \in \mathcal{D}\left(\Sigma_{\text {int }}\right)$ with $p \in \partial \mathcal{D}(\Sigma)$. However, we have $\partial \mathcal{D}(\Sigma)=H^{+}(\Sigma) \cup H^{-}(\Sigma)$ where $H^{+}$and $H^{-}$denote the future and past Cauchy horizons. Without loss of generality, we may assume $p \in H^{+}(\Sigma)$. Since $\Sigma$ is edgeless, $p$ lies on a past inextendible null geodesic $\lambda$ which remains in $H^{+}(\Sigma)$. However, since $p \in \mathcal{D}^{+}\left(\Sigma_{\text {int }}\right)$ and $\lambda$ does not intersect $\Sigma_{\text {int }}$, it follows that $\lambda \subset \mathcal{D}^{+}\left(\Sigma_{\text {int }}\right) \subset \mathcal{D}\left(\Sigma_{\text {int }}\right)$. Since $\mathcal{D}\left(\Sigma_{\text {int }}\right)$ is compact, this violates strong causality (cf. e.g. lemma 8.2.1 of [22]).

Similarly, we have

Lemma 3.6 Let $\left(M, g_{a b}\right)$ be a spacetime of class (b) and suppose that $\mathcal{D}\left(\Sigma_{\mathrm{int}}\right)$ is compact. Then $\mathcal{D}\left(\Sigma_{\text {int }}\right) \subset \stackrel{o}{\mathcal{D}}(\Sigma) \cup \mathcal{H}$.

We now prove the main theorems of this section.

Theorem 3.1 Let $\left(M, g_{a b}\right)$ be a spacetime of class (a). Then

$$
(\mathcal{B} \cup \mathcal{W}) \cap \stackrel{o}{\mathcal{D}}(\Sigma)=\emptyset
$$

if and only if

(i) $A_{i} A_{j}>0$ for all $i, j$, where the $A_{i}$ 's are the constants $A$ of definition 2.1 (cf. eq. (2.5)) in the $i$ 'th end, and

(ii) $\mathcal{D}\left(\Sigma_{\text {int }}\right)$ is compact.

Remark: It is easily seen that compactness of domains of dependence of compact sets can hold even if black hole and white hole regions occur when Killing orbits having different time orientations exist in $\left(M, g_{a b}\right)$ - an example is given by e.g. the slice $\Sigma^{\prime}$ in the space-time of Figure 1.1 from which the blocks $E$ and $F$ have been removed. (After these excisions the metric on this space-time can actually be taken to be that of the $|e|<m$ Reissner-Nordström space-time).

Proof of $(\mathcal{B} \cup \mathcal{W}) \cap \stackrel{o}{\mathcal{D}}(\Sigma)=\emptyset \Longrightarrow$ (i), (ii): That property (i) holds follows from lemma 3.2 applied to the spacetime $M^{\prime}=\stackrel{o}{\mathcal{D}}(\Sigma)$. We shall prove compactness of $\mathcal{D}\left(\Sigma_{\text {int }}\right) \cap \stackrel{o}{\mathcal{D}}(\Sigma)$, from which it follows by elementary topology considerations that $\mathcal{D}\left(\Sigma_{\text {int }}\right) \subset \stackrel{o}{\mathcal{D}}(\Sigma)$ and hence that $\mathcal{D}\left(\Sigma_{\text {int }}\right)$ is compact. To prove compactness of $\mathcal{D}\left(\Sigma_{\text {int }}\right) \cap \stackrel{o}{\mathcal{D}}(\Sigma)$, we consider a sequence $\left\{x_{i}\right\}$ in $\mathcal{D}\left(\Sigma_{\text {int }}\right) \cap \stackrel{o}{\mathcal{D}}(\Sigma)$ and will show existence of an accumulation point. By corollary 3.4 the orbit through $x_{i}, \gamma_{i} \equiv \bigcup_{t \in(-\infty, \infty)} \phi[X]_{t}\left(x_{i}\right)$, must intersect $\Sigma$ (possibly more than once). Let $y_{i}$ denote an intersection point of $\gamma_{i}$ with $\Sigma$, and let $t_{i}$ be such that $\phi[X]_{t_{i}}\left(y_{i}\right)=x_{i}$. It is easily seen, e.g. using the coordinates of Proposition 2.1 that we have $\mathcal{D}\left(\Sigma_{\text {int }}\right) \cap M_{\text {ext }}=\emptyset$, which implies $x_{i} \notin M_{\text {ext }}$, and hence $y_{i} \in \Sigma_{\text {int }}$. Since $\Sigma_{\text {int }}$ is compact, there exists a subsequence $\left\{\tilde{y}_{i}\right\}$ which converges to $y \in \Sigma_{\text {int }}$. Let $\left\{\tilde{t}_{i}\right\}$ denote the corresponding sequence of numbers satisfying $\phi[X]_{\tilde{t}_{i}}\left(\tilde{y}_{i}\right)=\tilde{x}_{i}$.

Now, by lemma 3.1 the orbit, $\gamma$, through $y$ satisfies $I^{+}(\gamma) \supset M_{j}$ for some $j$. Let $t_{-} \in \mathbb{R}$ be such that $I^{+}\left[\phi[X]_{t_{-}}(y)\right] \cap \Sigma_{\text {ext }} \neq \emptyset$. Then $I^{+}\left[\phi[X]_{t}(y)\right] \cap \Sigma_{\text {ext }} \neq \emptyset$ for all $t<t_{-}$. However, 
$I^{+}\left[\mathcal{D}\left(\Sigma_{\text {int }}\right)\right] \cap \Sigma_{\text {ext }}=\emptyset$, so $\phi[X]_{t}(y) \notin \mathcal{D}\left(\Sigma_{\text {int }}\right)$ for $t<t_{-}$. Similarly, there exists $t_{+} \in \mathbb{R}$ such that $\phi[X]_{t}(y) \notin \mathcal{D}\left(\Sigma_{\text {int }}\right)$ for all $t>t_{+}$. However, since $\phi[X]_{\tilde{t}_{i}}\left(\tilde{y}_{i}\right) \in \mathcal{D}\left(\Sigma_{\text {int }}\right)$ it follows by continuity of $\phi[X]_{t}(y)$ with respect to $t$ and $y$ that $\lim \sup \tilde{t}_{i} \leq t_{+}, \liminf \tilde{t}_{i} \geq t_{-}$. Thus, $\left\{\tilde{t}_{i}\right\}$ is a bounded sequence and hence has an accumulation point $t$. The point $x=\phi[X]_{t}(y)$ is then an accumulation point of the original sequence $\left\{x_{i}\right\}$. We have $x \in \mathcal{D}\left(\Sigma_{\text {int }}\right)$ since $\mathcal{D}\left(\Sigma_{\text {int }}\right)$ is closed, and we have $x \in \stackrel{o}{\mathcal{D}}(\Sigma)$ since all orbits in $\stackrel{o}{\mathcal{D}}(\Sigma)$ are complete (cf. Proposition 3.1 and the discussion following Proposition 3.2).

Proof of (i) and (ii) $\Longrightarrow(\mathcal{B} \cup \mathcal{W}) \cap \stackrel{o}{\mathcal{D}}(\Sigma)=\emptyset$ : Given (i), by changing time orientation if necessary we may assume without loss of generality that $A_{i}>0$ for all $i$. We restrict attention to the globally hyperbolic region $M^{\prime}=\stackrel{o}{\mathcal{D}}(\Sigma)$ which, by Proposition 3.1, also is a spacetime of class (a). By Lemma 3.5 and condition (ii), we have that $\mathcal{D}\left(\Sigma_{\text {int }}\right) \subset M^{\prime}$, so $\mathcal{D}\left(\Sigma_{\text {int }}\right)$ is compact as a subset of $M^{\prime}$. Furthermore, $\mathcal{B} \cap M^{\prime}$ is precisely the black hole region of the spacetime $M^{\prime}$, and similarly for $\mathcal{W} \cap M^{\prime}$. This means that, without loss of generality, we may assume that $M$ is globally hyperbolic with Cauchy surface $\Sigma$, and we then must prove that $\mathcal{B} \cup \mathcal{W}=\emptyset$.

Suppose, now, that $\mathcal{W} \neq \emptyset$. We note, first, that it follows straightforwardly from the definition of $\mathcal{B}$ and $\mathcal{W}$ that $\mathcal{B} \subset J^{+}\left[\mathcal{D}\left(\Sigma_{\text {int }}\right)\right]$ and $\mathcal{W} \subset J^{-}\left[\mathcal{D}\left(\Sigma_{\text {int }}\right)\right]$. Furthermore, $J^{-}[\mathcal{W}] \subset \mathcal{W}$. Let $x \in \mathcal{W}$ and consider a past inextendible causal curve $\lambda$ with future end point $x$. Clearly, we have $\lambda \subset \mathcal{W}$. Since $M$ is globally hyperbolic, $\lambda$ must enter and remain in $I^{-}(\Sigma)$. However, if $\lambda$ remained in the compact region $\mathcal{D}\left(\Sigma_{\text {int }}\right)$, we would violate strong causality. This implies that $\lambda$ must enter $I^{-}\left[\Sigma_{\text {ext }}\right]$ so that there exists a point $p \in \mathcal{W} \cap I^{-}\left[\Sigma_{\text {ext }}\right]$. Since $I^{-}\left[\Sigma_{\text {ext }}\right] \cap \mathcal{B}=\emptyset$ we have $p \notin \mathcal{B}$. Choose any $q \in I^{-}(p)$, and let $\tau_{0}>0$ denote the Lorentz distance, $\tau(q, p)$, between $q$ and $p$. Clearly, we also have $q \in \mathcal{W} \cap I^{-}\left[\Sigma_{\text {ext }}\right]$, and $q \notin \mathcal{B}$.

Consider, now, the orbit, $\gamma_{q}$, of $q$ under the isometries. Since $\mathcal{W}$ is isometry invariant, we have $\gamma_{q} \subset \mathcal{W} \subset J^{-}\left[\mathcal{D}\left(\Sigma_{\text {int }}\right)\right]$, so $\gamma_{q} \cap J^{+}\left[\Sigma_{\text {ext }}\right]=\emptyset$, i.e., for all $t \in \mathbb{R}$ we have $\phi[X]_{t}(q) \cap J^{+}\left[\Sigma_{\text {ext }}\right]=\emptyset$. On the other hand for each $j$ such that $I^{+}\left(\gamma_{q}\right) \cap M_{j} \neq \emptyset$, by lemma 3.1, we have $M_{j} \subset J^{+}\left[\gamma_{q}\right]$. Equivalently, for each $j$ such that $I^{+}\left(\gamma_{q}\right) \cap M_{j} \neq \emptyset, J^{+}(q)$ intersects every orbit in $M_{j}$, and in particular, $J^{+}(q)$ intersects every orbit on $\partial M_{j}$. Since $\partial \Sigma_{\text {ext }}$ has only a finite number of connected components each of which is compact, it follows that there exists $T \in \mathbb{R}$ such that $\phi[X]_{T}\left[J^{+}(q) \cap \partial M_{\text {ext }}\right] \subset J^{+}(\Sigma)$. This implies that for all $t \geq T$, we have $\phi[X]_{t}(q) \cap J^{-}\left[\Sigma_{\text {ext }}\right]=\emptyset$. Combined with the previous result, this shows that for all $t \geq T$ we have $\phi[X]_{t}(q) \in \mathcal{D}\left(\Sigma_{\text {int }}\right)$. Similarly, increasing $T$ if necessary, for all $t \geq T$ we have $\phi[X]_{t}(p) \in \mathcal{D}\left(\Sigma_{\text {int }}\right)$.

Now for $n \in \mathbb{N}$, consider the sequences $\left\{p_{n} \equiv \phi[X]_{n}(p)\right\},\left\{q_{n} \equiv \phi[X]_{n}(q)\right\}$. Since $p, q \notin \mathcal{B}$, we have $p_{n}, q_{n} \notin \mathcal{B}$ for all $n$. Since $\left\{p_{n}\right\}$ enters and remains in the compact subset $\mathcal{D}\left(\Sigma_{\text {int }}\right)$, there exists a subsequence $\left\{p_{n_{i}}\right\}$ which converges to a point $x \in \mathcal{D}\left(\Sigma_{\text {int }}\right)$. Similarly, the corresponding subsequence $\left\{q_{n_{j}}\right\}$ has a sub-subsequence $\left\{q_{n_{i_{j}}}\right\}$ converging to $y \in \mathcal{D}\left(\Sigma_{\text {int }}\right)$. By isometry invariance, we have $\tau\left(q_{n_{i_{j}}}, p_{n_{i_{j}}}\right)=\tau_{0}$. By continuity of the Lorentz distance function $\tau$ in a globally hyperbolic spacetime, we have $\tau(y, x)=\tau_{0}>0$. In particular this implies that $x \in I^{+}(y)$. However, since $x$ is in the $\omega$-limit set of the orbit of $p$, and $y$ is in the $\omega$-limit set of the orbit of $q$, by lemma 3.4 we have $x, y \in \mathcal{B}$. Since $q_{n_{i_{j}}}, p_{n_{i_{j}}} \notin \mathcal{B}$, we must have $x, y \in \partial \mathcal{B}$. However, $\partial \mathcal{B}$ is an achronal set, which contradicts $x \in I^{+}(y)$. The hypothesis that $\mathcal{B} \neq \emptyset$ leads to a contradiction in a similar manner.

Before proving the analogous theorem for spacetimes of class (b), we shall need the following lemma: 
Lemma 3.7 Let $\left(M, g_{a b}\right)$ be a spacetime of class (b) such that $(\mathcal{B} \cup \mathcal{W}) \cap \stackrel{o}{\mathcal{D}}(\Sigma)=\emptyset$. Then, for $p \in \mathcal{H}_{+}$, the $\alpha$-limit set, $\alpha_{p}$, of the orbit $\phi[X]_{t}(p)$ is a nonempty subset of $S$. Similarly for $p \in \mathcal{H}_{-}, \omega_{p}$ is a nonempty subset of $S$.

Remark: Note that the proof below shows that the null geodesic generators of $\mathcal{H}_{+}$are actually future complete. [This follows from the fact that for each $n \in \mathbb{N}$ the minimum value of affine parameter on the cross-section $\phi[X]_{n t_{+}}\left[S^{+}\right]$is $\left.\beta^{n} \epsilon\right]$.

Proof: Since by Lemma 3.4, any $\alpha$-limit set is contained in $\mathcal{W}$, and since $\mathcal{H}_{+} \backslash S$ is contained in $I^{+}[\stackrel{o}{\mathcal{D}}(\Sigma)]$, it follows immediately that the $\alpha$-limit set of any orbit on $\mathcal{H}_{+}$must be a subset of $S$. Thus, we need only prove that this subset is nonempty. To do so, on $S$, define $k^{a}=n^{a}+r^{a}$, where $n^{a}$ is the future-directed unit normal to $\Sigma$ and $r^{a}$ is the unit normal to $S$ in $\Sigma$ (pointing "towards $\Sigma$ "). Then $k^{a}$ is tangent to the null geodesic generators of $\mathcal{H}_{+}$. [This can be seen as follows: Clearly, $I^{+}[S] \cap \mathcal{D}(\Sigma)$ is empty, so the null geodesic determined by $k^{a}$ can't lie in $\stackrel{o}{\mathcal{D}}(\Sigma)$. On the other hand, a simple local argument can be used to show that sufficiently near $S$, this null geodesic lies in $\mathcal{D}(\Sigma)$.] Let $\lambda$ denote the affine parameter along the null generators of $\mathcal{H}_{+}$determined by $k^{a}$. Consider the map, $\psi$, defined perhaps only on a sufficiently small neighborhood, $\mathcal{O} \subset S \times \mathbb{R}^{+}$, of $S \times\{0\}$ which takes $(p, \lambda)$ into the point lying at affine parameter $\lambda$ on the null generator of $\mathcal{H}_{+}$through $p \in S$. By decreasing $\mathcal{O}$ if necessary, we may assume that this map is a diffeomorphism of $\mathcal{O}$ onto a neighborhood of $S$ in $\mathcal{H}_{+}$. Consequently, we may choose $\epsilon>0$ such that $\psi[S, \epsilon]$ is a smooth cross-section, $S^{+}$, of $\mathcal{H}_{+}$. Decreasing $\epsilon$ if necessary, we may assume that $S^{+} \subset \mathcal{D}\left(\Sigma_{\text {int }}\right)$.

Let $p \in S^{+}$. Then there exists $q \in \stackrel{o}{\mathcal{D}}(\Sigma)$ such that $q \in I^{-}(p)$. Since $\mathcal{W} \cap \stackrel{o}{\mathcal{D}}(\Sigma)=\emptyset$, by Corollary 3.4 there exists $t_{q}$ such that for $s \geq t_{q}$ we have $\phi[X]_{s}(q) \in I^{+}\left(\Sigma_{\text {ext }}\right)$. Set $t_{p}=t_{q}$, so that for all $s \geq t_{p}$ we have $\phi[X]_{s}(p) \in I^{+}\left(\Sigma_{\text {ext }}\right)$. Since $I^{+}\left(\Sigma_{\text {ext }}\right)$ is open, there exist open neighbourhods $\mathcal{U}_{p}$ of $\phi[X]_{2 t_{p}}(p)$ satisfying $\mathcal{U}_{p} \subset I^{+}\left(\Sigma_{\text {ext }}\right)$. Set $\mathcal{O}_{p}=\phi[X]_{-2 t_{p}}\left(\mathcal{U}_{p}\right) \cap S^{+}$. It follows (passing to open subsets of $\mathcal{U}_{p}$ if necessary) that $\left\{\mathcal{O}_{p}\right\}_{p \in S^{+}}$is a covering of $S^{+}$by open sets such that the implication $\left[q \in \mathcal{O}_{p} \Rightarrow \forall s \geq 2 t_{p} \phi[X]_{s}(q) \in I^{+}\left(\Sigma_{\text {ext }}\right)\right]$ holds. By compactness of $S^{+}$a finite covering $\left\{\mathcal{O}_{p_{i}}\right\}_{i=1}^{N}$ can be chosen. Set $t_{+}=2 \max _{i} t_{p_{i}}$, define

$$
S_{t}^{+} \equiv \phi[X]_{t}\left(S^{+}\right)
$$

By construction we have $S_{t_{+}}^{+} \subset I^{+}\left(\Sigma_{\text {ext }}\right)$. Let $\alpha$ be the minimum value of affine parameter on $S_{t_{+}}^{+}$. Then, clearly $\alpha>\epsilon$.

Now, for all $t, \phi[X]_{t}$ maps affinely parametrized null geodesic generators of $\mathcal{H}_{+}$into affinely parametrized null geodesic generators of $\mathcal{H}_{+}$. In particular, the above argument shows that $\phi[X]_{t_{+}}$maps each null geodesic segment on $\mathcal{H}_{+}$with past endpoint on $S$ and of affine length $\epsilon$ into a null geodesic segment on $\mathcal{H}_{+}$with past endpoint on $S$ and of affine length at least $\beta \epsilon$, where $\beta=\alpha / \epsilon>1$. It follows that $\phi[X]_{t_{+}}$maps any null geodesic segment on $\mathcal{H}_{+}$starting at $S$ and having affine length $\lambda$ into a similar null geodesic segment of affine length at least $\beta \lambda$. It then follows immediately that $\phi[X]_{n t_{+}}$maps each such null geodesic segment of affine length $\lambda$ into a null geodesic segment of affine length at least $\beta^{n} \lambda$. Conversely, $\phi[X]_{-n t_{+}}$maps each such null geodesic segment of affine length $\lambda$ into a similar null geodesic segment of affine length no larger than $\beta^{-n} \lambda$.

Now, let $p \in \mathcal{H}_{+}$. Consider the sequence $\phi[X]_{-n t_{+}}(p)$. Let $\left\{p_{n}\right\} \in S$ denote the intersection with $S$ of the generator of $\mathcal{H}_{+}$through $\phi[X]_{-n t_{+}}$. Compactness of $S$ implies existence of a 
subsequence which converges to $q \in S$. It then follows immediately that $q$ is an $\alpha$-limit set of the orbit through $p$, so the $\alpha$-limit set of $\phi[X]_{t}(p)$ is nonempty, as we desired to show.

The theorem for spacetimes of class (b) analogous to Theorem 3.1 is the following:

Theorem 3.2 Let $\left(M, g_{a b}\right)$ be a spacetime of class (b). Then

$$
(\mathcal{B} \cup \mathcal{W}) \cap \stackrel{o}{\mathcal{D}}(\Sigma)=\emptyset
$$

if and only if

(i) $A_{i} A_{j}>0$ for all $i, j$, where the $A_{i}$ 's are the constants $A$ of definition 2.1 (cf. eq. (2.5)) in the $i$ 'th end, and

(ii) $\mathcal{D}\left(\Sigma_{\text {int }}\right)$ is compact.

Remark: As shown in the first paragraph of the first part of the proof below, under the hypothesis that $(\mathcal{B} \cup \mathcal{W}) \cap \stackrel{o}{\mathcal{D}}(\Sigma)=\emptyset$, we have $\mathcal{B} \cap M^{\prime}=\mathcal{H}_{+}$and $\mathcal{W} \cap M^{\prime}=\mathcal{H}_{-}$. This implies that $\mathcal{H}_{+}$comprises (a portion of) the event horizon of the black hole, $\mathcal{H}_{-}$comprises (a portion of) the white hole horizon, and that the intersection of the black and white hole horizons is $S$. Thus, spacetimes of class (b) which satisfy the hypothesis of theorem 3.2 correspond to the spacetimes of "class (2)" discussed in the Introduction.

Proof of $(\mathcal{B} \cup \mathcal{W}) \cap \stackrel{o}{\mathcal{D}}(\Sigma)=\emptyset \Longrightarrow$ (i), (ii): That property (i) holds follows from lemma 3.3 . To prove (ii), we first note that by corollary 3.3, we have $S \subset(\mathcal{B} \cap \mathcal{W})$. Since $J^{+}(\mathcal{B}) \subset \mathcal{B}$, we have $\mathcal{H}_{+} \subset \mathcal{B}$. However, we must have $\left(\mathcal{H}_{-} \backslash S\right) \cap \mathcal{B}=\emptyset$, since otherwise we would have $\mathcal{B} \cap \stackrel{o}{\mathcal{D}}(\Sigma) \neq \emptyset$. This implies that $\mathcal{B} \cap M^{\prime}=\mathcal{H}_{+}$, where $M^{\prime} \equiv \stackrel{o}{\mathcal{D}}(\Sigma) \cup \mathcal{H}$. Similarly, we have $\mathcal{W} \cap M^{\prime}=\mathcal{H}_{-}$.

We shall prove compactness of $\mathcal{D}\left(\Sigma_{\text {int }}\right) \cap M^{\prime}$ from which it follows by elementary topology that $\mathcal{D}\left(\Sigma_{\text {int }}\right) \subset M^{\prime}$ and hence that $\mathcal{D}\left(\Sigma_{\text {int }}\right)$ is compact. Let $\left\{x_{i}\right\}$ be an infinite sequence in $\mathcal{D}\left(\Sigma_{\text {int }}\right) \cap M^{\prime}$. We wish to show existence of an accumulation point. Without loss of generality, we may assume that infinitely many of the $x_{i}$ lie in $\mathcal{D}^{+}\left(\Sigma_{\text {int }}\right) \cap M^{\prime}$.

Let $X^{\prime}$ be a vector field on $M$ which is transverse to $\Sigma$, (strictly) timelike on a neighbourhood of $\Sigma_{\text {int }}$ and is such that $X^{\prime}=X$ in $M_{\text {ext }}$, at least for $r$ large enough. Since $\Sigma_{\text {int }}$ is compact, there exists $\epsilon>0$ such that the induced action $\phi\left[X^{\prime}\right]_{t}(\Sigma)$ generated by $X^{\prime}$ for $t \in(-2 \epsilon, 2 \epsilon)$ yields a spacelike foliation, such that $M^{\prime} \cap \mathcal{O}$ is a closed subset of the spacetime region $\mathcal{O}$ covered by this foliation and such that $X^{\prime}$ is timelike in $\left(I^{+}\left(\Sigma_{\text {int }}\right) \cup I^{-}\left(\Sigma_{\text {int }}\right)\right) \cap \mathcal{O}$. We denote the hypersurface at $t=\epsilon$ by $\widetilde{\Sigma}$. Clearly $\widetilde{\Sigma} \subset I^{+}(\Sigma)$, and the intersection of $\widetilde{\Sigma}$ with $\mathcal{H}$ yields a cross-section of $\mathcal{H}_{+} \backslash S$. We denote by $\mathcal{R}$ the region covered by the foliation for $t \in[0, \epsilon]$. We denote by $\mathcal{R}_{\text {int }} \subset \mathcal{R}$ the portion of $\mathcal{R}$ connected to $\Sigma_{\text {int }}$ by integral curves of $X^{\prime}$. Finally, we write $\widetilde{\Sigma}_{\text {int }}=\widetilde{\Sigma} \cap \mathcal{R}_{\text {int }}$ and note that the construction ensures that $J^{-}\left(\widetilde{\Sigma}_{\text {int }}\right) \cap \mathcal{D}^{+}\left(\Sigma_{\text {int }}\right) \cap M^{\prime} \subset \mathcal{R}_{\text {int }}$ and $\mathcal{D}\left(\Sigma_{\text {int }}\right) \cap M^{\prime} \backslash \mathcal{R}_{\text {int }} \subset J^{+}\left(\widetilde{\Sigma}_{\text {int }}\right) \cap M^{\prime}$.

Now $\mathcal{R}_{\text {int }}$ is compact, since it has topology $[0, \epsilon] \times \Sigma_{\text {int }}$. Since both $\mathcal{D}^{+}\left(\Sigma_{\text {int }}\right)$ and $M^{\prime} \cap \mathcal{R}$ are closed, $\mathcal{D}^{+}\left(\Sigma_{\text {int }}\right) \cap M \cap \mathcal{R}_{\text {int }}$ is compact. Hence, $\left\{x_{i}\right\}$ must have an accumulation point unless all but finitely many elements of this sequence lie in $\mathcal{D}^{+}\left(\Sigma_{\text {int }}\right) \cap M^{\prime} \backslash \mathcal{R}_{\text {int }}$. Hence, without loss of generality, we may assume that $\left\{x_{i}\right\}$ is a sequence in $\mathcal{D}^{+}\left(\Sigma_{\text {int }}\right) \cap M^{\prime} \cap J^{+}\left(\widetilde{\Sigma}_{\text {int }}\right)$. For 
each $i$, either $x_{i} \in \stackrel{o}{\mathcal{D}}(\Sigma) \cap J^{+}\left(\widetilde{\Sigma}_{\text {int }}\right)$ or $x_{i} \in \mathcal{H}_{+}$. If $x_{i} \in \stackrel{o}{\mathcal{D}}(\Sigma)$, then by hypothesis $x_{i} \notin \mathcal{B}$ and by the same type of argument as in theorem [3.1, the half- orbit $\gamma_{i}=\cup_{(-\infty, 0]} \phi[X]_{t}\left(x_{i}\right)$ must intersect $\widetilde{\Sigma}$ in the compact set $\widetilde{\Sigma}_{\text {int }} \cap M^{\prime}$. On the other hand, if $x_{i} \in \mathcal{H}_{+}$, using lemma 3.7, it follows immediately that the similar half-orbit $\gamma_{i}$ also intersects $\widetilde{\Sigma}$ in $\widetilde{\Sigma}_{\text {int }} \cap M^{\prime}$. Since $\mathcal{W} \cap\left(\widetilde{\Sigma}_{\text {int }} \cap M^{\prime}\right)=\emptyset$, existence of an accumulation point for $\left\{x_{i}\right\}$ follows by an argument similar to that used in the proof of the corresponding part of theorem 3.1.

Proof of (i) and (ii) $\Longrightarrow(\mathcal{B} \cup \mathcal{W}) \cap \stackrel{o}{\mathcal{D}}(\Sigma)=\emptyset$ : By Lemma 3.6 we have $\mathcal{D}\left(\Sigma_{\text {int }}\right) \subset \stackrel{o}{\mathcal{D}}(\Sigma) \cup \mathcal{H}$. As in the proof of theorem 3.1, we assume that $\mathcal{W} \cap \stackrel{o}{\mathcal{D}}(\Sigma) \neq \emptyset$ and obtain a contradiction. Let $x \in \mathcal{W} \cap \stackrel{o}{\mathcal{D}}(\Sigma)$ and let $\lambda$ be a causal curve through $x$ which is past inextendible in $\stackrel{o}{\mathcal{D}}(\Sigma)$. Then either $\lambda$ is past inextendible in $\stackrel{o}{\mathcal{D}}(\Sigma) \cup \mathcal{H}$ or $\lambda$ has a past endpoint on $\mathcal{H}_{-}$. In the latter case, we have $x \in I^{+}(\gamma)$, where $\gamma$ is a null geodesic generator of $\mathcal{H}_{-}$on which $\lambda$ has a past endpoint. We then may choose a causal curve $\lambda^{\prime}$ through $x$ which lies in $I^{+}(\gamma) \cap I^{-}(x) \subset \stackrel{o}{\mathcal{D}}(\Sigma)$ and is past inextendible in $M$ (cf. lemma 8.1 .4 of [22]). Thus, in either case, we obtain a causal curve in $\stackrel{o}{\mathcal{D}}(\Sigma)$ which is past inextendible in $\stackrel{o}{\mathcal{D}}(\Sigma) \cup \mathcal{H}$. By strong causality, this curve cannot lie entirely within $\mathcal{D}\left(\Sigma_{\text {int }}\right)$, and we thereby obtain a point $p \in \mathcal{W} \cap \stackrel{o}{\mathcal{D}}(\Sigma)$ with $p \notin \mathcal{B}$. The remainder of the proof parallels the proof of the corresponding portion of theorem 3.1 .

We end this Section with some additional results. First, for any set $K \subset M$ (where $K$ is not necessarily achronal) we define the "effective domain of dependence" of $K$, denoted $\tilde{\mathcal{D}}(K)$, to consist of all $p \in M$ such that every (past and future) inextendible timelike curve through $p$ intersects $K$. We then have the following:

Corollary 3.5 Let $\left(M, g_{a b}\right)$ be a space-time of class (a), suppose that

$$
(\mathcal{B} \cup \mathcal{W}) \cap \stackrel{o}{\mathcal{D}}(\Sigma)=\emptyset
$$

For any compact $K \subset \stackrel{o}{\mathcal{D}}(\Sigma), \tilde{\mathcal{D}}(K)$ is compact.

Proof: Suppose first that $K \subset \Sigma$. One can enlarge $K$ or $\Sigma_{\text {int }}$ or both so that $K=\Sigma_{\text {int }}$ holds, and compactness of $\tilde{\mathcal{D}}(K)=\mathcal{D}(K)$ follows by Theorem 3.1.

In the general case, let $p \in K$. By Corollary 3.4 there exists $t_{p}^{ \pm}$such that $\phi[X]_{t_{p}^{ \pm}}(p) \in$ $I^{ \pm}\left(\Sigma_{\text {ext }}\right)$. Since $I^{ \pm}\left(\Sigma_{\text {ext }}\right)$ is open, there exist open neighbourhods $\mathcal{V}_{p}^{ \pm}$of $\phi[X]_{t_{ \pm}}(p)$ satisfying $\mathcal{V}_{p}^{ \pm} \subset I^{ \pm}\left(\Sigma_{\text {ext }}\right)$. Set $\mathcal{O}_{p}=\phi[X]_{-t_{p}^{ \pm}}\left(\mathcal{V}_{p}^{ \pm}\right) \cap K$. It follows that $\left\{\mathcal{O}_{p}\right\}_{p \in K}$ is a covering of $K$ by open sets satisfying $\left[q \in \mathcal{O}_{p} \Rightarrow \phi[X]_{t_{p}^{ \pm}}(q) \in I^{ \pm}(\Sigma)\right]$; by compactness of $K$ a finite covering $\left\{\mathcal{O}_{p_{i}}\right\}_{i=1}^{N}$ can be chosen. Let $t_{ \pm}=\max _{i}\left\{-t_{p_{i}}^{\mp}\right\}$; it follows that $K \subset I^{\mp}\left(\phi[X]_{t_{ \pm}}(\Sigma)\right)$. By Proposition 3.1 $\phi[X]_{t_{ \pm}}(\Sigma)$ are Cauchy surfaces, so that by [13] $K^{ \pm} \equiv J^{ \pm}(K) \cap \phi[X]_{t_{ \pm}}(\Sigma)$ are compact. Now $\tilde{\mathcal{D}}^{ \pm}(K)$ are closed subsets of $\mathcal{D}^{ \pm}\left(K^{\mp}\right)$, which have already been shown to be compact, and the result follows.

Corollary 3.6 Let $\left(M, g_{a b}\right)$ be a space-time of class (b), suppose that

$$
(\mathcal{B} \cup \mathcal{W}) \cap \stackrel{\circ}{\mathcal{D}}(\Sigma)=\emptyset
$$

For any compact $K \subset \stackrel{o}{\mathcal{D}}(\Sigma) \cup \mathcal{H}, \tilde{\mathcal{D}}(K)$ is compact. 
Proof: It suffices to prove compactness of $J^{+}(\Sigma) \cap \tilde{\mathcal{D}}(K)$. Enlarging $\Sigma_{\text {int }}$ if necessary we may assume $K \cap M_{\text {ext }}=\emptyset$. Let $\mathcal{R}, \tilde{\Sigma}$, etc., be as in the proof of Theorem 3.2, set $K_{0}=K \cap \mathcal{R}$, $K_{+}=K \cap J^{+}(\tilde{\Sigma})$. Arguing as in the proof of Corollary 3.5 there exists $t_{+}$such that $K_{+} \subset$ $\cup_{t \in\left[0, t_{+}\right]} \phi[X]_{t}(\tilde{\Sigma})$. Replacing $\mathcal{R}$ by $\mathcal{R} \cup_{t \in\left[0, t_{+}\right]} \phi[X]_{t}(\tilde{\Sigma})$ and $\tilde{\Sigma}$ by $\phi[X]_{t_{+}}(\tilde{\Sigma})$ if necessary we may without loss of generality assume $t_{+}=0$. Now the argument of the proof of Theorem 3.2 shows that $\tilde{\mathcal{D}}\left(\mathcal{R}_{\text {int }}\right)$ is compact, and the result follows since $\tilde{\mathcal{D}}(K)$ is a closed subset of $\tilde{\mathcal{D}}\left(\mathcal{R}_{\text {int }}\right)$.

Let us remark that it follows immediately from the inclusion

$$
M \backslash \stackrel{o}{\mathcal{D}}(\Sigma) \subset \mathcal{B} \cup \mathcal{W}
$$

proved in Corollary 3.1 that a space-time of class (a) in which $\mathcal{B} \cup \mathcal{W}=\emptyset$ is globally hyperbolic. We shall end this Section by proving a related result:

Proposition 3.4 Let $\left(M, g_{a b}\right)$ be a space-time of class (a), and suppose that

$$
\left(\mathcal{B}_{1} \cup \mathcal{W}_{1}\right) \cap \stackrel{o}{\mathcal{D}}(\Sigma)=\emptyset
$$

where $\mathcal{B}_{1}$ and $\mathcal{W}_{1}$ are the black hole region and the white hole region with respect to a given end $M_{1}$. Then

$$
M=\stackrel{o}{\mathcal{D}}(\Sigma)
$$

In other words, $\stackrel{\circ}{\mathcal{D}}(\Sigma)$ is inextendible in the class of space-times of class (a).

\section{Remarks:}

1. If $M$ has only one end, then clearly (3.2) is equivalent to $(\mathcal{B} \cup \mathcal{W}) \cap \stackrel{o}{\mathcal{D}}(\Sigma)=\emptyset$. It is, however, possible to have (3.2) and more than one end, as is e.g. shown by the metric $-d t^{2}+g_{i j} d x^{i} d x^{j}$, where $g_{i j} d x^{i} d x^{j}$ is the space-part of the "Schwarzschild wormhole" metric (two asymptotically flat ends connected by a throat). Other examples of this kind (with an arbitrary number of ends) can be constructed by appropriately gluing together along boundaries of world-tubes space-times of class (a) in which the Killing vector is timelike everywhere.

2. The following example shows that the hypothesis of strong causality of the potential extensions is necessary in general: Consider the space-time $M^{\prime}$ consisting of blocks $B, D$ and $E$ of Figure 1.1, let $t$ be a Killing time function on $M$ as given by Proposition 4.1 below, let $\Sigma_{0}$ be the zero level set of $t$; use $t$ to identify $M^{\prime}$ with $\mathbb{R} \times \Sigma_{0}$. On $\Sigma_{0}$ remove two balls $B_{a} \subset \Sigma_{0} \cap M_{a}, a=1,2$, and identify $\left(t, p_{1}\right), p_{1} \in \partial B_{1}$ with $\left(t, p_{2}\right), p_{2}=\phi\left(p_{1}\right) \in \partial B_{2}$, where $\phi$ is a smooth diffeomorphism from $\partial B_{1}$ to $\partial B_{2}$. Smoothing out the metric near $\mathbb{R} \times \partial B_{a}, a=1,2$ one obtains a space-time $M$ with a Killing vector field which has complete orbits. Note that $t$ passes from $M^{\prime}$ to the quotient space-time $M$ and therefore $M$ is stably causal, thus $M$ is a space-time of class (a). Clearly $M$ is extendible because $M^{\prime}$ is, and the identifications which lead to the construction of $M$ are done only in the interior of $M^{\prime}$.

3. Let us point out that neither the hypothesis of existence of Killing vectors in the (potential) extended space-time, nor the the hypothesis of stable causality of the (potential) 
extended space-time are needed to show inextendibility of $M$ when the Killing vector is strictly timelike. For suppose that $\left(M, g_{a b}\right)$ were extendible to a space-time $\left(M^{\prime}, g_{a b}^{\prime}\right)$ with a $C^{2}$ metric $g_{a b}^{\prime}$, it follows from equation (2.6) that $X$ can be extended to a continuous vector field defined on the closure $\bar{M}$ of $M$ in $M^{\prime}$. Now $\partial M$ is an achronal topological hypersurface, so that every timelike vector is necessarily transverse to $\partial M$. Let $p \in \partial M$, if $X(p)$ were timelike the orbits of $X$ could not be complete in $M$, hence $g_{a b} X^{a} X^{b}\left(p_{i}\right)$ must tend to zero on any sequence $p_{i} \in M$ such that $p_{i} \rightarrow p$. But the hypothesis that $X$ is strictly timelike on $M$ together with asymptotic flatness imply that there exists $\epsilon>0$ such that $g_{a b} X^{a} X^{b}<-\epsilon$ on $M$, and the result follows.

Proof: We note first that $\left(\mathcal{B}_{1} \cup \mathcal{W}_{1}\right) \cap \stackrel{o}{\mathcal{D}}(\Sigma)=\emptyset$ implies that for each $i$, we have $I^{-}\left(M_{i}\right) \supset \stackrel{o}{\mathcal{D}}(\Sigma)$, since by Lemma 3.1 we have $I^{-}\left(M_{i}\right) \supset M_{1}$, and, hence, $I^{-}\left(M_{i}\right) \supset I^{-}\left(M_{1}\right) \supset \stackrel{o}{\mathcal{D}}(\Sigma)$. Similarly, for each $i$ we have $I^{+}\left(M_{i}\right) \supset \stackrel{o}{\mathcal{D}}(\Sigma)$.

Suppose, now, that $M \neq \stackrel{o}{\mathcal{D}}(\Sigma)$. Then $H \neq \emptyset$, where $H$ denotes the Cauchy horizon of $\Sigma$. Without loss of generality, we may assume that $H^{+} \neq \emptyset$. Let $p \in H^{+}$. By theorem 3.1 and Lemma 3.5, we have $p \notin \mathcal{D}^{+}\left(\Sigma_{\text {int }}\right)$, and, hence, $p \in I^{+}\left(\Sigma_{\text {ext }}\right)$. Replacing $\Sigma$ by $\phi[X]_{t}(\Sigma)$ in this argument, we see that for all $t \in \mathbb{R}$ we have $p \in I^{+}\left(\phi[X]_{t}\left[\Sigma_{\text {ext }}\right]\right)$. However, this implies that $I^{-}(p) \supset M_{i}$ for some $i$, and, hence, $I^{-}(p) \supset I^{-}\left(M_{i}\right) \supset \mathcal{D}(\Sigma)$.

Now, let $\mathcal{O}$ be any open neighborhood of $p$ which does not intersect $M_{\text {ext }}$, and let $\mathcal{V}$ be any open neighborhood of $p$ contained in $\mathcal{O}$. Since $p$ lies on the boundary of $\stackrel{o}{\mathcal{D}}(\Sigma)$, there exists $q \in \mathcal{D}(\Sigma) \cap \mathcal{V}$. Since $q \notin \mathcal{B}$, there exists a future-directed causal curve $\lambda_{1}$ from $q$ to a point $r \in M_{\text {ext }}$. Since $I^{-}(p) \supset \mathcal{D}(\Sigma)$, there exists a future-directed causal curve $\lambda_{2}$ from $r$ to $p$. Clearly, the causal curve $\lambda=\lambda_{1} \cup \lambda_{2}$ has more than one connected component in $\mathcal{V}$, thus showing that strong causality is violated at $p$.

The above proof caries over immediately to spacetimes of class (b), in the following sense:

Proposition 3.5 Let $\left(M, g_{a b}\right)$ be a space-time of class (b), and suppose that

$$
\left(\mathcal{B}_{1} \cup \mathcal{W}_{1}\right) \cap \stackrel{o}{\mathcal{D}}(\Sigma)=\emptyset
$$

where $\mathcal{B}_{1}$ and $\mathcal{W}_{1}$ are the black hole region and the white hole region with respect to a given end $M_{1}$. Then $H=\mathcal{H}$, where $H$ denotes the Cauchy horizon of $\Sigma$.

\section{Maximal Slicings}

The main results of this section, and indeed, of this paper, are the following:

Theorem 4.1 Let $\left(M, g_{a b}\right)$ be a space-time of class (a) and suppose that

$$
(\mathcal{B} \cup \mathcal{W}) \cap \stackrel{o}{\mathcal{D}}(\Sigma)=\emptyset
$$

Then $\stackrel{o}{\mathcal{D}}(\Sigma)$ can be covered by a family of maximal (spacelike) asymptotically flat slices $\hat{\Sigma}_{s}$, $s \in \mathbb{R}$, which are Cauchy surfaces for $\stackrel{\circ}{\mathcal{D}}(\Sigma)$, such that

$$
\phi[X]_{t}\left(\hat{\Sigma}_{s}\right)=\hat{\Sigma}_{s+t}, \quad \text { and } \quad \hat{\Sigma}_{s} \approx \Sigma,
$$


where $\approx$ means "diffeomorphic to". If moreover $X$ is timelike on $\stackrel{\circ}{\mathcal{D}}(\Sigma)$ or if the timelike convergence condition holds,

$$
R_{a b} Z^{a} Z^{b} \geq 0 \text { for all timelike } Z^{a},
$$

then $\left\{\hat{\Sigma}_{t}\right\}_{t \in \mathbb{R}}$ foliates $\stackrel{o}{\mathcal{D}}(\Sigma)$.

Theorem 4.2 Let $(M, g)$ be a space-time of class (b) and suppose that (4.1) holds. Then $\stackrel{o}{\mathcal{D}}(\Sigma)$ can be covered by a family of maximal (spacelike) asymptotically flat hypersurfaces $\hat{\Sigma}_{s}$, $s \in \mathbb{R}$, with boundary $\partial \hat{\Sigma}_{s}=\partial \Sigma=S$, such that (4.9) holds. Moreover $\hat{\Sigma}_{s} \backslash S$ are Cauchy surfaces for $\stackrel{\circ}{\mathcal{D}}(\Sigma)$.

If moreover $X$ is timelike on $\stackrel{\circ}{\mathcal{D}}(\Sigma)$ or if the timelike convergence condition (4.⿹) holds, then $\left\{\hat{\Sigma}_{t} \backslash S\right\}_{t \in \mathbb{R}}$ foliates $\stackrel{o}{\mathcal{D}}(\Sigma)$.

\section{Remarks.}

1. Theorem 4.1 generalizes a similar Theorem proved in [5], where strict stationarity (the Killing vector timelike everywhere) is assumed.

2. As mentioned in Corollary 4.4 of [5], the existence of maximal slices restricts the allowed topologies of $\Sigma$; thus there exist no space-times satisfying the hypotheses of Theorem 4.1 and the timelike convergence condition (4.3) for which $\Sigma$ 's topology satisfies a genericity condition.

3. It should be noted that, even though asymptotically flat, the maximal hypersurfaces will in general not be asymptotic to the original $t$ slicing of the exterior region. Even if $\alpha=1$, where $\alpha$ is the fall-off rate of the metric in the asymptotic region, $c f$. Definition 2.1, one will only have $|u| \leq C(1+\ln (1+r))$, where $u$ is the "height function" of $\hat{\Sigma}_{0}$ in $M_{\text {ext }}$.

4. If the timelike convergence condition holds, one has uniqueness of $\left\{\hat{\Sigma}_{t}\right\}_{t \in \mathbb{R}}$ under some supplementary rather weak conditions [5] [Theorem 5.5]. Proposition 1 and Theorem 1 of [10] can be used to remove the "boost-domain" conditions of [5] [Theorem 5.5] if, e.g., the metric satisfies some hyperbolic field equations for which the "boost problem" ( $c f$. [9]) is well posed.

Before passing to the proofs of our results, let us present in some detail an example which shows that the hypothesis $(\mathcal{B} \cup \mathcal{W}) \cap \stackrel{o}{\mathcal{D}}(\Sigma)=\emptyset$ of Theorem 4.1 cannot be removed without imposing some other conditions: Let $\left(\hat{M}, \hat{g}_{a b}\right)$ be the maximally analytically extended Schwarzschild space-time, with "stationary" Killing vector $X$. Let $\hat{\Sigma}_{0}$ be the standard "Einstein-Rosen bridge" maximal surface extending from one end $\hat{M}_{1}$ to the other end $\hat{M}_{2}$. Let $t$ denote the Killing time function based on $\hat{\Sigma}_{0}$, defined in the ends $\hat{M}_{a}, a=1,2$. Consider any complete, asymptotically flat, maximal hypersurface $\hat{\Sigma}$ in $\hat{M}$ which is asymptotically orthogonal to the Killing vector. With a little work one shows that there exist $t_{1}, t_{2} \in \mathbb{R}$ such that $\hat{\Sigma}$ is asymptotic to the level sets $t=t_{a}$ in $\hat{M}_{a}, a=1,2$. This together with the uniqueness results in [5] implies that $\hat{\Sigma}$ is necesssarily spherically symmetric. 
We let $\Omega$ consist of the union of the causal future of the "north pole" $p \in S \equiv\{r \in \hat{M} \mid X(r)=$ $0\}$ with the causal past of the "south pole" $q \in S$ of the bifurcation sphere $S$. Consider the space-time $\left(M, g_{a b}\right)$ defined as $M \equiv \hat{M} \backslash \Omega, g_{a b}=\left.\hat{g}_{a b}\right|_{M}$. As $\Omega$ is invariant under the flow of $X$ in $\hat{M}$, so is $M$, hence all the Killing orbits in $M$ are complete. "Pushing" $\hat{\Sigma}_{o}$ slightly to the future in a neighbourhood of $q$ and slightly to the past in a neighbourhood of $p$ one obtains a complete asymptotically flat hypersurface $\Sigma_{0}$ in $M$, thus $M$ is of class (a). Moreover $M$ has both a black hole and a white hole region, thus $M$ does not satisfy the hypotheses of Theorem 4.1. Let $\Sigma$ be any complete, asymptotically flat, maximal surface in $M$ which is asymptotically orthogonal to $X$. It follows that such a surface is also a maximal surface in $\hat{M}$ which enjoys the same properties. By what has been said above it follows that $\Sigma$ must be spherically symmetric. But it is clear from e.g. the Penrose diagram for the maximally extended Schwarzschild space-time that there are no spherically symmetric complete hypersurfaces in $M$. Consequently, there are no complete, asymptotically flat, maximal surfaces in $M$ which are asymptotically orthogonal to $X$, and sharpness of Theorem 4.1 follows.

The proofs of Theorems 4.1 and 4.2 will largely run in parallel. The idea is to construct some appropriate time functions (given in Propositions 4.1 and 4.2 below), and to prove a height estimate by moving the surfaces by the isometry group. Higher order a priori estimates follow then from Bartnik's work [2], and existence is obtained by standard arguments. Note that from Theorems 3.1 and 3.2, it follows that, without loss of generality we may assume that for spacetimes of class (a) and (b) the Killing orbits in $M_{\text {ext }}$ are future oriented.

If the Killing field $X$ were timelike throughout $M$, the desired time functions for cases (a) and (b) could be constructed very simply as the parameter along the integral curves of $X$ starting at $\Sigma$; i.e. by solving $\mathcal{L}_{X} t=1$ with the initial condition $t=0$ on $\Sigma$. However, in case (a), $X$ need not be timelike in $M_{\text {int }}$, so $X$ need not be transverse to $\Sigma$, in which case this simple construction does work. The situation is worse in case (b), since the condition $\phi[X]_{t}[S]=S$ implies that no time function $t$ can satisfy $\mathcal{L}_{X} t=1$ throughout $\mathcal{D}(\Sigma)$. Nevertheless, we shall show that in case (a) an asymptotically flat slice, $\Sigma^{\prime}$ (which is a Cauchy surface for $\stackrel{o}{\mathcal{D}}(\Sigma)$ ) can be constructed which is everywhere transverse to $X$. Similarly, in case (b) an asymptotically flat hypersurface, $\Sigma^{\prime}$ with boundary $S$ can be constructed such that $\Sigma^{\prime} \backslash S$ is transverse to $X$ and is a Cauchy surface for $\stackrel{o}{\mathcal{D}}(\Sigma)$. These results will enable us to prove the following propositions:

Proposition 4.1 Let $\left(M, g_{a b}\right)$ be a space-time of class (a), suppose that

$$
(\mathcal{B} \cup \mathcal{W}) \cap \stackrel{o}{\mathcal{D}}(\Sigma)=\emptyset
$$

Then there exists a time function $\tau \in C^{\infty}(\stackrel{o}{\mathcal{D}}(\Sigma))$ with asymptotically flat level sets $\Sigma_{s}(\tau)=$ $\{p: \tau(p)=s\}$ (which are Cauchy surfaces for $\stackrel{\circ}{\mathcal{D}}(\Sigma)$ ) such that $\phi[X]_{t}$ acts by translations in $\tau$ :

$$
\phi[X]_{t}\left(\Sigma_{s}(\tau)\right)=\Sigma_{s+t}(\tau) .
$$

Proposition 4.2 Let $\left(M, g_{a b}\right)$ be a space-time of class (b), suppose that (4.4) holds. Then there exists a neighbourhood $\mathcal{U}$ of $\stackrel{\circ}{\mathcal{D}}(\Sigma) \cup \mathcal{H}$ and a time function $\tilde{\tau} \in C^{\infty}(\mathcal{U})$, with asymptotically flat level sets $\Sigma_{s}(\tilde{\tau})=\{p: \tilde{\tau}(p)=s\}$ such that

$$
\phi[X]_{t}\left(\Sigma_{s}(\tilde{\tau}) \cap \mathcal{D}(\Sigma)\right)=\Sigma_{s+t}(\tilde{\tau}) \cap \mathcal{D}(\Sigma)
$$


for $s \geq s_{0}, t \geq 0$ and for $s \leq-s_{o}, t \leq 0$, for some $s_{0}$. Moreover, for all $t, s \in \mathbb{R}$

$$
\phi[X]_{t}\left(\Sigma_{s}(\tilde{\tau}) \cap M_{\text {ext }}\right)=\Sigma_{s+t}(\tilde{\tau}) \cap M_{\text {ext }} .
$$

Furthermore, $\Sigma_{0}(\tilde{\tau}) \cap \stackrel{o}{\mathcal{D}}(\Sigma)$ is a Cauchy surface for $\stackrel{o}{\mathcal{D}}(\Sigma)$ and $\partial\left\{\Sigma_{0}(\tilde{\tau}) \cap \mathcal{D}(\Sigma)\right\}=S$.

Remark: If the timelike convergence condition (4.3) holds, for spacetimes of class (b), a somewhat shorter though certainly less elegant proof of Proposition 4.2 can be given. The relevant argument is outlined in the proof of Theorem B.1, Appendix B.

Before beginning the proofs of Propositions 4.1 and 4.2, we introduce the following notation. For any hypersurface $\hat{\Sigma} \subset M$ we set

$$
\begin{aligned}
& \hat{\Sigma}_{0, \mathrm{ext}}=\hat{\Sigma}_{\mathrm{ext}}=\hat{\Sigma} \cap M_{\mathrm{ext}} \\
& \hat{\Sigma}_{t, \mathrm{ext}}=\phi[X]_{t}\left(\hat{\Sigma}_{\mathrm{ext}}\right) \\
& \hat{\Sigma}_{0, \mathrm{int}}=\hat{\Sigma}_{\mathrm{int}}=\hat{\Sigma} \cap M_{\mathrm{int}} \\
& \hat{\Sigma}_{t, \mathrm{int}}=\phi[X]_{t}\left(\hat{\Sigma}_{\mathrm{int}}\right)
\end{aligned}
$$

and

$$
\begin{gathered}
\delta \leq 0, \quad \hat{\Sigma}_{0, \delta}=\left\{p \in \hat{\Sigma}_{\text {int }}: d_{\hat{\Sigma}}\left(p, \partial \hat{\Sigma}_{\text {int }}\right) \geq|\delta|\right\} \\
\delta \geq 0, \quad \hat{\Sigma}_{0, \delta}=\hat{\Sigma}_{\text {int }} \cup_{i=1}^{I}\left\{p \in \hat{\Sigma}_{i}: R_{i} \leq r(p) \leq R_{i}+\delta\right\},
\end{gathered}
$$

where $d_{\hat{\Sigma}}\left(p, \partial \hat{\Sigma}_{\text {int }}\right)$ is the Riemannian distance on $\hat{\Sigma}$ from $p$ to $\partial \hat{\Sigma}_{\text {int }}, r(p)$ is the coordinate radius function in the end under consideration, and the $R_{i}$ 's are the constants $R_{1}$ of Definition 2.1 in the $i$ 'th end. There exists $\epsilon_{0}>0$ such that for $\delta>-\epsilon_{0}$ the sets $\hat{\Sigma}_{0, \delta}$ are manifolds with smooth boundary.

Proof of proposition 4.1: Let $(t, x)$ be coordinates on $M_{\text {ext }}$ as given by Proposition 2.1, normalized so that $A=1$ in all the ends, $M_{i}$, and so that $g_{\mu \nu}$ is asymptotically conformal (with constant conformal factor) to the standard Minkowski metric. Let $\stackrel{o}{g}$ be any smooth Lorentzian metric on $M$ which, coincides with $g$ on $M_{\text {int }}$, and such that

$$
\stackrel{o}{g}=-d t^{2}+\sum_{i=1}^{3}\left(d x^{i}\right)^{2}
$$

for all $x$ such that $r(x) \geq R$, for some $R \geq R_{i}, i=1, \ldots, I$, on all ends $M_{i}$. Let $\tilde{X}$ be any smooth vector field defined on a neighborhood of $\Sigma$, which is transverse to $\Sigma$, and which coincides with $X$ for $r(x) \geq R$. On $M_{\text {ext }}$ define

$$
\rho=\underset{g}{\square_{g}} t
$$

where $\square_{g}$ is the d'Alembertian of the metric $\stackrel{o}{g}$. Let $\rho_{1} \in C^{\infty}(M)$ be any function satisfying $\rho_{1}=\rho$ for $r \geq R$. Define $t_{1} \in C^{\infty}(\stackrel{o}{\mathcal{D}}(\Sigma))$ as the unique solution of the Cauchy problem

$$
\begin{aligned}
\square_{g} t_{1} & =\rho_{1}, \\
\left.t_{1}\right|_{\Sigma} & =0, \\
\left.\left(\mathcal{L}_{\widetilde{X}} t_{1}\right)\right|_{\Sigma} & =1,
\end{aligned}
$$


Let $\widetilde{\mathcal{O}} \subset \stackrel{o}{\mathcal{D}}(\Sigma)$ be defined by $\widetilde{\mathcal{O}}=\left\{p \in \stackrel{o}{\mathcal{D}}(\Sigma): \nabla t_{1}\right.$ is timelike $\}$. Let $\mathcal{O} \subset \widetilde{\mathcal{O}}$ be the domain of dependence of $\Sigma$ in the space-time $\left(\widetilde{\mathcal{O}}, g_{a b}\right)$. Lie dragging along integral curves of $\nabla t_{1}, \mathcal{O}$ can be identified with $\left\{\left(t_{1}, p\right) \in \mathbb{R} \times \Sigma: t^{-}(p)<t_{1}<t^{+}(p)\right\}$ for some functions $t^{ \pm}(p) \in \mathbb{R} \cup\{ \pm \infty\}$. Compactness of $\Sigma_{0,2 R}$ implies that there exists $\epsilon>0$ such that $(-4 \epsilon, 4 \epsilon) \times \Sigma_{0,2 R} \subset \mathcal{O}$. From uniqueness of solutions of the problem (4.7) it follows that for $\left(t_{1}, x\right) \in \Omega=\{r \geq R,|t| \leq r-R\}$ we have $t_{1}=t$. Decreasing $\epsilon$ if necessary we thus have $\mathcal{O}_{4 \epsilon} \subset \mathcal{O}$, where $\mathcal{O}_{s}=(-s, s) \times \Sigma=$ $\left\{p:\left|t_{1}(p)\right|<s\right\}$. Further decreasing $\epsilon$ if necessary, we have $t_{1}=t$ at all points in $\mathcal{O}_{4 \epsilon}$ with $r>2 R$.

Next, we claim that for all $p \in \stackrel{o}{\mathcal{D}}(\Sigma)$, the set $\left\{t \in \mathbb{R}: \phi[X]_{t}(p) \in \mathcal{O}_{2 \epsilon}\right\}$ is nonempty and bounded. Namely, nonemptiness is an immediate consequence of Corollary 3.4. To prove boundedness, we note that if the orbit of $X$ through $p$ intersects $\Sigma$ at $r>2 R$ in $\Sigma_{\text {ext }}$, then boundedness follows immediately from the fact that $t_{1}=t$ at all points in $\mathcal{O}_{2 \epsilon}$ with $r>2 R$. Hence, if $\phi[X]_{t_{i}}(p) \in \mathcal{O}_{2 \epsilon}$ for an unbounded sequence $\left\{t_{i}\right\}$, then infinitely many of these points lie in the compact set $[-2 \epsilon, 2 \epsilon] \times \Sigma_{0,2 R}$. Passing to a convergent subsequence, we obtain a nonempty $\alpha$-limit set or $\omega$-limit set (or both). However, this contradicts Lemma 3.4 .

The desired time function $\tau$ may now be constructed as follows: Let $t_{2}$ be the Lipschitz continuous, piecewise smooth function defined on $M$ by

$$
t_{2}(p)= \begin{cases}t_{1}(p), & p \in \mathcal{O}_{2 \epsilon} \\ 2 \epsilon, & p \in I^{+}(\Sigma) \backslash \mathcal{O}_{2 \epsilon} \\ -2 \epsilon, & p \in I^{-}(\Sigma) \backslash \mathcal{O}_{2 \epsilon}\end{cases}
$$

Let $\varphi \in C^{\infty}(\mathbb{R})$ be any function satisfying

$$
\varphi(x)= \begin{cases}0, & x \leq-\epsilon \\ 1, & x \geq \epsilon\end{cases}
$$

$\left.\varphi^{\prime}(x)\right|_{(-\epsilon, \epsilon)}>0$, and

$$
\varphi(x)-\frac{1}{2}=-\left(\varphi(-x)-\frac{1}{2}\right)
$$

Define

$$
t_{3}=\varphi \circ t_{2}
$$

It follows that $t_{3} \in C^{\infty}(M)$ with

$$
t_{3}(p)= \begin{cases}0, & p \in I^{-}(\Sigma) \backslash \mathcal{O}_{\epsilon} \\ 1, & p \in I^{+}(\Sigma) \backslash \mathcal{O}_{\epsilon}\end{cases}
$$

and we also have

$$
\nabla t_{3}(p)= \begin{cases}0, & p \in M \backslash \mathcal{O}_{\epsilon} \\ \text { timelike, past pointing, } & p \in \mathcal{O}_{\epsilon} .\end{cases}
$$

For $p \in \stackrel{o}{\mathcal{D}}(\Sigma)$ we define

$$
\tau(p)=\int_{-\infty}^{0} t_{3}\left(\phi[X]_{s}(p)\right) d s+\int_{0}^{\infty}\left(t_{3}-1\right)\left(\phi[X]_{s}(p)\right) d s .
$$

We now claim: 


$$
\text { for } \quad p \in \stackrel{o}{\mathcal{D}}(\Sigma), \quad|\tau(p)|<\infty \text {. }
$$

Indeed let $p \in \stackrel{o}{\mathcal{D}}(\Sigma)$. Then, as shown above, there exists $t_{ \pm},\left|t_{ \pm}\right|<\infty$, such that for $t \geq t_{+}$ $t_{3}\left(\phi[X]_{t}(p)\right)=1$, and for $t \leq t_{-} t_{3}\left(\phi[X]_{t}(p)\right)=0$, so that the equation (4.11) reads

$$
\tau(p)=\int_{t_{-}}^{0} t_{3}\left(\phi[X]_{s}(p)\right) d s+\int_{0}^{t_{+}}\left(t_{3}-1\right)\left(\phi[X]_{s}(p)\right) d s
$$

which proves (4.12). Standard results about integrals with parameters and (4.13) imply $\tau \in$ $C^{\infty}(\stackrel{o}{\mathcal{D}}(\Sigma))$.

2)

$$
X(\tau)=1
$$

Namely, by a change of variables we have

$$
\tau\left(\phi[X]_{t}(p)\right)=\int_{-\infty}^{t} t_{3}\left(\phi[X]_{s}(p)\right) d s+\int_{t}^{\infty}\left(t_{3}-1\right)\left(\phi[X]_{s}(p)\right) d s,
$$

so that

$$
\left.X(\tau)\right|_{p}=\left.\frac{d}{d t} \tau\left(\phi[X]_{t}(p)\right)\right|_{t=0}=1 .
$$

[Let us note, that (4.14) implies that for $p \in \stackrel{o}{\mathcal{D}}(\Sigma)$, we have $X_{p} \neq 0$ (cf. also Corollary 3.2)].

$$
\nabla \tau \quad \text { is timelike on } \stackrel{o}{\mathcal{D}}(\Sigma)
$$

Namely, we have

$$
\nabla \tau(p)=\int_{-\infty}^{\infty}\left(\phi[X]_{s_{*}} \nabla t_{3}\right)(p) d s,
$$

so that (4.15) follows from $(4.10)$ and from the fact, that for any isometry $\psi$ defined on a connected set the tangent map $\psi_{*}$ maps timelike, consistently oriented vectors to timelike, consistently oriented vectors.

4)

$$
\text { the level sets of } \tau \text { are asymptotically flat. }
$$

To prove (4.16), consider $p=(t, x) \in\{r \geq R+\epsilon,|t| \leq r-R\}$, with $R, \epsilon$ as at the beginning of the proof of this Proposition. For such $p$ we have $t_{3}\left(\phi[X]_{s}(p)\right)=\phi[X](s+t)$, so that $\partial \tau / \partial x^{i}=0$, thus $\tau=\tau(t)$. (4.14) gives $\tau=t+\tau_{0}$ for some constant $\tau_{0}$. The symmetry condition (4.8) gives $\tau_{0}=0$, so that in $\{r \geq R+\epsilon,|t| \leq r-R\} \tau$ coincides with the original Killing time $t$.

5)

the level sets of $\tau$ are Cauchy surfaces for $\stackrel{\circ}{\mathcal{D}}(\Sigma)$. 
To prove (4.17), we note first that (4.15) implies that any level surface of $\tau$ is achronal. Consider the restriction of $\tau$ to the original slice $\Sigma$. By the previous argument, we have $\left.\tau\right|_{\Sigma}=0$ for all $r>R$, so $\left.\tau\right|_{\Sigma}$ can be nonvanishing only on a compact subset of $\Sigma$, and, hence, there exists $T \in \mathbb{R}$ such that $|\tau|<T$ on $\Sigma$. Applying $\phi[X]_{T}$ to this result (using (4.14)), we see that $\tau>0$ everywhere on $\Sigma_{T}$. Similarly, we have $\tau<0$ on $\Sigma_{-T}$. However, by Proposition 3.1, $\Sigma_{T}$ and $\Sigma_{-T}$ are Cauchy surfaces for $\stackrel{o}{\mathcal{D}}(\Sigma)$. Hence, every inextendible timelike curve in $\stackrel{o}{\mathcal{D}}(\Sigma)$ intersects $\Sigma_{T}$ and $\Sigma_{-T}$, and, thus, passes from positive to negative values of $\tau$. By continuity, every such inextendible timelike curve intersects the level set $\tau=0$, thus implying that this level set is a Cauchy surface for $\stackrel{o}{\mathcal{D}}(\Sigma)$. That all the other level sets of $\tau$ also are Cauchy surfaces for $\stackrel{o}{\mathcal{D}}(\Sigma)$ then follows by application of Proposition 3.1.

The above results complete the proof of Proposition 4.1.

In order to prove Proposition 4.2, we first shall need the following Lemma:

Lemma 4.1 Let $\left(M, g_{a b}\right)$ be a space-time of class (b) and suppose that (4.4) holds. Then there exists a smooth spacelike hypersurface $\Sigma_{0}$ with boundary $S$ such that $\Sigma_{0}$ coincides with $\Sigma$ outside of a compact set, $\Sigma_{0} \cap \stackrel{o}{\mathcal{D}}(\Sigma)$ is a Cauchy surface for $\stackrel{\circ}{\mathcal{D}}(\Sigma)$, and $X$ is everywhere transverse to $\Sigma_{0} \backslash S$

Proof: The idea of the proof is to construct a time function $\tau$ in $\stackrel{o}{\mathcal{D}}(\Sigma)$ in a way somewhat similar to that in the proof of Proposition 4.1. Supplementary difficulties arise because we need to ensure that the hypersurface $\Sigma_{0}$ defined by $\tau=0$ smoothly intersects $S$ and remains spacelike "up to boundary". Thus, the proof of the Lemma will consist mainly of constructing a function $t_{1}$ [cf. eq. (4.7)] near $S$ so that $\Sigma_{0}$ will have the desired properties there.

Let $N$ denote the normal bundle of $S$, i.e., a point of $N$ consists of a point $p \in S$ together with a vector $V^{a}$ at $p$ which is normal to $S$. As in the proof of Lemma 3.7, let $n^{a}$ denote the future-directed unit normal field to $\Sigma$ on $S$, and let $r^{a}$ denote the inward ("towards $\Sigma$ ") pointing unit normal to $S$ in $\Sigma$. Then at each $p \in S,\left(n^{a}, r^{a}\right)$ is a basis for normal vectors to $S$ at $p$, so we can uniquely express each normal vector, $V^{a}$, at $p$ as $V^{a}=T n^{a}+Z r^{a}$. Thus, each $x \in N$ is uniquely characterized by $(p, T, Z)$ with $p \in S$ and $T, Z \in \mathbb{R}$.

Consider the "wedge", $\mathcal{R}$, of $N$ defined by $Z>|T|$. It is convenient to introduce "Rindler coordinates" $\left(t^{\prime}, z^{\prime}\right)$ in $\mathcal{R}$ by,

$$
\begin{aligned}
t^{\prime} & =\tanh ^{-1}(T / Z), \\
z^{\prime} & =\sqrt{Z^{2}-T^{2}}
\end{aligned}
$$

so that $z^{\prime}$ is just the length of the normal vector to $S$. Now, since $\phi[X]_{t}$ maps $S$ into $S$ and maps vectors normal to $S$ into vectors normal to $S$, it induces an action on $N$, which we shall denote by $\phi^{\prime}[X]_{t}$. Since $\phi[X]_{t}$ preserves the length of vectors normal to $S$, it follows immediately that on $\mathcal{R}$, the action of $\phi^{\prime}[X]_{t}$ takes the form,

$$
\phi^{\prime}[X]_{t}\left(p, t^{\prime}, z^{\prime}\right)=\left(\phi[X]_{t}(p), t^{\prime}+f_{t}(p), z^{\prime}\right)
$$

for some function $f_{t}$ on $S$. Furthermore, it follows directly from the proof of Lemma 3.7 that there exists $a>0$ such that we have $f_{t_{+}}(p)>a$ for all $p \in S$, where $t_{+}$was defined in Lemma 3.7. Consequently, for all $n \in \mathbb{N}$ we have $f_{n t_{+}}(p)>n a$ for all $p \in S$, from which it follows 
that $f_{t}(p) \rightarrow \infty$ as $t \rightarrow \infty$ and $f_{t}(p) \rightarrow-\infty$ as $t \rightarrow-\infty$. In other words, along every orbit, $\phi^{\prime}[X]_{t}$, in $\mathcal{R}$, we have $t^{\prime} \rightarrow \infty$ as $t \rightarrow \infty$ and $t^{\prime} \rightarrow-\infty$ as $t \rightarrow-\infty$.

We now repeat the construction used to obtain the time function $\tau$ of Proposition 4.1, replacing $\stackrel{o}{\mathcal{D}}(\Sigma)$ by $\mathcal{R}$, replacing $\phi[X]_{t}$ by $\phi^{\prime}[X]_{t}$ and replacing the time function $t_{1}$ (defined in a neighborhood of $\Sigma$ in $\stackrel{o}{\mathcal{D}}(\Sigma)$ by eq. (4.7)) by the function $t^{\prime}$ (which is globally well defined in $\mathcal{R}$, so we choose an arbitrary $\epsilon>0$ to define the analog, $t_{2}^{\prime}$, of $\left.t_{2}\right)$. From what has been said at the end of the previous paragraph it follows that the resulting function, denoted $\tau^{\prime}$, will be well defined smooth on $\mathcal{R}$ (although it will be singular on the boundary of $\mathcal{R}$ ). Furthermore, it follows directly from the construction that throughout $\mathcal{R}$, we have $\partial \tau^{\prime} / \partial t^{\prime}>0$ and $\partial \tau^{\prime} / \partial z^{\prime}=0$, where the partial derivatives are taken holding $p$ fixed. (The first relation is the analog of property (3) in Proposition 4.1, while the second relation follows from the fact that both $t^{\prime}$ and the action of $\phi^{\prime}[X]_{t}\left[c f\right.$. eq. (4.20)] are independent of $z^{\prime}$.) It follows from the above properties of $\tau^{\prime}$ that in $\mathcal{R}$, the level surface, $\Sigma_{0}^{\prime}$, defined by $\tau^{\prime}=0$ is given by an equation of the form $t^{\prime}=g(p)$, where $\mathrm{g}$ is a smooth function on $S$. However, returning to the original, globally nonsingular coordinates $(T, Z)$ on $N$, we see immediately that any hypersurface of this form in $\mathcal{R}$ can be smoothly extended to a hypersurface $\widetilde{\Sigma}_{0}^{\prime}$ through $S$, and the magnitude of the "slope" of $\widetilde{\Sigma}_{0}^{\prime}$ at $S$ satisfies $|\partial T / \partial Z|<1$.

Now, let $\psi: N \rightarrow M$ be the map which takes each $\left(p, V^{a}\right) \in N$ to the point in $M$ lying at unit affine parameter along the geodesic in $M$ determined by the initial conditions $\left(p, V^{a}\right)$. Then, by the same arguments as used for the ordinary exponential map, it follows that $\psi$ is a diffeomorphism from some open neighborhood, $\mathcal{U}$, of $S$ in $N$ to an open neighborhood, $\mathcal{V}$ of $S$ in $M$. In particular, the image, $\widetilde{\Sigma}_{0}$, under $\psi$ of, $\widetilde{\Sigma}_{0}^{\prime}$ is a smooth hypersurface through $S$ in $M$. Furthermore, the above "slope" condition implies that $\widetilde{\Sigma}_{0}$ is spacelike at $S$.

The pullback under $\psi^{-1}$ of the function $t^{\prime}$ on $\mathcal{R} \cap \mathcal{U}$ defines a smooth function, $\hat{t}$, on $\stackrel{o}{\mathcal{D}}(\Sigma) \cap \mathcal{V}$. In $\stackrel{o}{\mathcal{D}}(\Sigma) \cap \mathcal{V}$, we define the function $\hat{\rho}$ by

$$
\hat{\rho}=\nabla^{a} \nabla_{a} \hat{t}
$$

Note that from the definition of $t^{\prime}$, it follows immediately that in a neighborhood of $S$ on $\Sigma$, we have $\hat{\lambda} \equiv n^{a} \nabla_{a} \hat{t}>0$, where $n^{a}$ here denotes the unit normal to $\Sigma$; indeed, we have $\hat{\lambda} \rightarrow \infty$ in the limit as $S$ is approached.

As in the proof of Proposition 1.1, let $(t, x)$ be coordinates on $M_{\text {ext }}$ as given by Proposition 2.1, normalized so that $A=1$ in all the ends and so that $g_{\mu \nu}$ is asymptotically conformal (with constant conformal factor) to the standard Minkowski metric. Again, we let $\stackrel{o}{g}$ be any smooth Lorentzian metric on $M$ which, coincides with $g$ on $M_{\text {int }}$, and such that

$$
\stackrel{o}{g}=-d t^{2}+\sum_{i=1}^{3}\left(d x^{i}\right)^{2}
$$

for all $x$ such that $r(x) \geq R$, for some $R \geq R_{i}, i=1, \ldots, I$, on all ends $M_{i}$. Let $\hat{X}$ be any smooth vector field defined on a neighborhood of $\Sigma$, which is transverse to $\Sigma$, and which coincides with $X$ for $r(x) \geq R$ and which coincides with the unit normal, $n^{a}$, to $\Sigma$ on a neighborhood of $S$ in $\Sigma$. Again, on $M_{\text {ext }}$ define

$$
\rho=\square_{g} t
$$


where $\square_{g}$ is the d'Alembertian of the metric $\stackrel{o}{g}$. Let $\hat{\rho}_{1} \in C^{\infty}(M)$ be any function satisfying $\hat{\rho}_{1}=\rho$ for $r \geq R$ and $\hat{\rho}_{1}=\hat{\rho}$ in the intersection of $\stackrel{o}{\mathcal{D}}(\Sigma)$ with some open neighborhood of $S$. Let $\hat{\lambda}_{1} \in C^{\infty}(\Sigma \backslash S)$ be any function satisfying $\hat{\lambda}_{1}>0, \hat{\lambda}_{1}=1$ for $r \geq R$ and $\hat{\lambda}_{1}=\hat{\lambda}$ in in the intersection of $\Sigma \backslash S$ with some open neighborhood of $S$. Let $\hat{\sigma}_{1} \in C^{\infty}(\Sigma \backslash S)$ be any function satisfying $\hat{\sigma}_{1}=0$ for $r \geq R$ and $\hat{\sigma}_{1}=\hat{t}$ in in the intersection of $\Sigma \backslash S$ with some open neighborhood of $S$. Define $\hat{t}_{1} \in C^{\infty}(\stackrel{o}{\mathcal{D}}(\Sigma))$ as the unique solution of the Cauchy problem

$$
\begin{aligned}
\square_{g} \hat{t}_{1} & =\hat{\rho}_{1}, \\
\left.\hat{t}_{1}\right|_{\Sigma} & =\hat{\sigma}_{1}, \\
\left.\left(\mathcal{L}_{\hat{X}} \hat{t}_{1}\right)\right|_{\Sigma} & =\hat{\lambda}_{1},
\end{aligned}
$$

Then $\hat{t}_{1}$ coincides with $\hat{t}$ in the intersection of $\stackrel{o}{\mathcal{D}}(\Sigma)$ with some neighborhood of $S$. On the neighbourhood $\mathcal{U}$ of $S$ defined above we have two families of maps defined, $\phi_{t}[X]$ and $\phi_{t}^{\prime}[X]$, and we claim that these maps coincide, wherever this statement makes sense. This follows from the fact that $\phi_{t}[X]$ maps geodesics starting at $S$ to geodesics starting at $S$, and preserves the geodesic distance along them. Consequently, the corresponding function $\hat{\tau}$ in $\stackrel{o}{\mathcal{D}}(\Sigma)$ obtained by the construction of Proposition 4.1 will coincide in a sufficiently small neighborhood of $S$ with the pullback under $\psi^{-1}$ of the function $\tau^{\prime}$ on $N$ constructed above. It then follows that the level surface $\hat{\tau}=0$ can be smoothly extended to $S$ so as to obtain a hypersurface with boundary satisfying all of the conditions of the Lemma.

Proof of Proposition 4.2: Let $\Sigma_{0}$ be as in Lemma 4.1, and let $\widetilde{\Sigma}$ be any smooth, spacelike extension of $\Sigma_{0}$ through $S$. Let $\tilde{n}^{a}$ denote the vector field - defined in a neighborhood, $\widetilde{\mathcal{O}}$, of $\widetilde{\Sigma}-$ of tangents to the geodesics normal to $\widetilde{\Sigma}$. In $\widetilde{\mathcal{O}} \cup M_{\text {ext }}$, define the vector field $\widetilde{X}$ by

$$
\tilde{X}^{a}=\chi \tilde{n}^{a}+(1-\chi) X^{a}
$$

where $\chi$ is any nonnegative, smooth function on $M$ such that $\chi=1$ in $M_{\text {int }}$ and for $r \leq R$ in $M_{\text {ext }}$ and $\chi=0$ for $r \geq 2 R$, where $R$ is defined as in the proof of Proposition 4.1. Then $\tilde{X}$ is transverse to $\widetilde{\Sigma}$, and by the same arguments as in the proof of the first part of Theorem 3.2 , there exists $\epsilon>0$ such that the induced action $\phi[\tilde{X}]_{\tilde{t}}$ yields a spacelike foliation of an open neighborhood of $\Sigma_{0}$ for $\tilde{t} \in(-2 \epsilon, 2 \epsilon)$. By decreasing $\epsilon$ if necessary, we can ensure that the hypersurfaces $\widetilde{\Sigma}_{ \pm \epsilon}$, defined by $t= \pm \epsilon$, intersect $\mathcal{H}$ in cross-sections of $\mathcal{H}_{+}$and $\mathcal{H}_{-}$, respectively. By further decreasing $\epsilon$ if necessary, we can ensure that $\widetilde{\Sigma}_{ \pm \epsilon} \cap M_{\text {int }}$ lies outside the causal future of the portion of $\widetilde{\Sigma}$ with $r \geq 2 R$.

We claim, now, that the hypersurfaces $\widetilde{\Sigma}_{ \pm \epsilon}$ are transverse to $X$ in an open neighborhood of $D(\Sigma)=D\left(\Sigma_{0}\right)$. Namely, in $M_{\text {ext }}$, this is obvious because $X$ is timelike. In $M_{\text {int }}$, the vector field $\tilde{n}^{a}$ is normal to $\widetilde{\Sigma}_{ \pm \epsilon}$. However, $X_{a} \tilde{n}^{a}$ is the inner product of a Killing field with a geodesic tangent, and thus is constant along the geodesics tangent to $\tilde{n}^{a}$. For any point $q \in \widetilde{\Sigma}_{ \pm \epsilon} \cap M_{\text {int }}$ the normal geodesic through $q$ is a timelike curve and, hence, must intersect $\widetilde{\Sigma}$ in $\Sigma_{0} \backslash S$. Since $X$ is transverse to $\Sigma_{0} \backslash S$, we have $X_{a} \tilde{n}^{a}<0$ everywhere on $\Sigma_{0}$, and, hence, $X_{a} \tilde{n}^{a}<0$ everywhere on $\widetilde{\Sigma}_{ \pm \epsilon} \cap D\left(\Sigma_{0}\right)$. This implies that $X$ is transverse to $\widetilde{\Sigma}_{ \pm \epsilon}$ in an open neighborhood of $D(\Sigma)$.

It follows immediately that the action of $\phi[X]_{\tilde{t}-\epsilon}$ on $\widetilde{\Sigma}_{\epsilon}$ for $\tilde{t}>\epsilon$ yields a foliation. As a consequence of Corollary 3.4 and Lemma 3.7, this foliation covers an open region containing $\left[I^{+}\left(\widetilde{\Sigma}_{\epsilon}\right)\right] \cap[\mathcal{D}(\Sigma) \cup \mathcal{H}]$. On the other hand, the transversality of $X$ to $\widetilde{\Sigma}_{\epsilon}$ and the tangency of 
$X$ to $\mathcal{H}$ imply that no point in $\left[J^{-}\left(\widetilde{\Sigma}_{\epsilon}\right)\right] \cap[\stackrel{o}{\mathcal{D}}(\Sigma) \cup \mathcal{H}]$ is covered by this foliation. Similarly, the action of $\phi[X]_{\tilde{t}+\epsilon}$ on $\widetilde{\Sigma}_{-\epsilon}$ for $\tilde{t}<-\epsilon$ yields a foliation covering an open region containing $\left[I^{-}\left(\widetilde{\Sigma}_{-\epsilon}\right)\right] \cap[\stackrel{o}{\mathcal{D}}(\Sigma) \cup \mathcal{H}]$ but containing no point in $\left[J^{+}\left(\widetilde{\Sigma}_{-\epsilon}\right)\right] \cap[\stackrel{o}{\mathcal{D}}(\Sigma) \cup \mathcal{H}]$. Consequently, by merging these foliations with the foliation defined by $\tilde{X}$ for $\tilde{t} \in[-\epsilon, \epsilon]$, we obtain a foliation covering an open region containing $\stackrel{o}{\mathcal{D}}(\Sigma) \cup \mathcal{H}$. The time function, $\tilde{t}$, of this foliation is easily verified to satisfy all the properties of this Proposition with one exception: although $\tilde{t}$ is continuous, it is only piecewise smooth in that it need not be smooth on $\widetilde{\Sigma}_{ \pm \epsilon}$ when $r>2 R$. However, since this possible non-smoothness of $\tilde{t}$ within $D(\tilde{\Sigma})$ occurs only on a set which intersects $D(\Sigma)$ compactly, we may smooth it e.g., by convolution with localized Friedrich mollifiers) to obtain a time function $\tilde{\tau}$ satisfying all the conditions of the Proposition.

Before proving Theorems 4.1 and 4.2 we prove the following Lemma.

Lemma 4.2 Let $\left(M, g_{a b}\right)$ be a space-time of class (a) or (b), let $t$ be a time function defined in a globally hyperbolic neighbourhood $\mathcal{O}$ of $\Sigma$, such that

1. $\Sigma$ is a Cauchy surface for $\mathcal{O}$, and

2. there exist constants $\theta \in[0,1), T \in \mathbb{R}$ such that for all $i=1, \ldots, I$,

$$
\mathcal{O} \cap M_{i} \subset \Omega_{\theta, R_{i}, T}=\left\{r \geq \widetilde{R},|t|<\theta\left(r-R_{i}\right)+T\right\},
$$

3. and $\left.t\right|_{\mathcal{O} \cap M_{\mathrm{ext}}}$ coincides with the Killing time based on $\Sigma \cap M_{\mathrm{ext}}$, as defined in Proposition 2.1, for $r \geq R$ for some $R$.

Let $\Sigma_{1} \subset \mathcal{O}$ be a smooth, embedded, acausal hypersurface which is a graph of a function $u_{1}$ over $\Sigma$ :

$$
\Sigma_{1}=\left\{p \in \mathcal{O}: t(p)=u_{1}(q), q \in \Sigma\right\},
$$

If $\left(M, g_{a b}\right)$ is of class (b), suppose moreover that $\left.u_{1}\right|_{S}=0$. Then $\Sigma_{1}$ is a Cauchy surface for $\stackrel{o}{\mathcal{D}}(\Sigma)$.

Proof: We can identify $\mathcal{O}$ with $\left\{(t, q): t_{-}(q)<t<t_{+}(q), q \in \Sigma\right\}$, for some functions $t_{ \pm} \in \mathbb{R} \cup\{ \pm \infty\}$, by dragging $q \in \Sigma$ along the integral curves of $\nabla t$. Let $\lambda$ be a piecewise differentiable inextendible causal curve in $\left(\mathcal{O}, g_{a b}\right)$, so $\lambda:\left(s_{-}, s_{+}\right) \ni s \rightarrow(s, \gamma(s)) \in \mathcal{O}$ for some piecewise differentiable curve $\gamma:\left(s_{-}, s_{+}\right) \rightarrow \Sigma$. Since $\Sigma$ is a Cauchy surface for $\mathcal{O}$ we have $\lambda \cap \Sigma \neq \emptyset$, and hence $0 \in\left(s_{-}, s_{+}\right)$. For any $\theta \in(0,1)$ one can choose $\mathcal{R}(\theta)$ large enough such that for $p \in M_{i}, i=1, \ldots, i$ and $r(p) \geq \mathcal{R}(\theta)$ the slopes of the light cones, in the coordinates of Proposition 2.1, lie between $\frac{1+\theta}{2}$ and $\frac{3-\theta}{2}$, which easily implies that there exists $C$ such that $\gamma\left(s_{-}, s_{+}\right) \subset \Sigma_{0, C}$. Consider a sequence $s_{i} \rightarrow s_{-}$. Compactness of $\Sigma_{0, C}$ implies that there exists $q_{-} \in \Sigma_{0, C}$ and a subsequence, still denoted by $s_{i}$, such that $\gamma\left(s_{i}\right) \rightarrow q_{-}$. Suppose that $s_{-} \neq t_{-}\left(q_{-}\right)$. Then the timelike curve $\left(t_{-}\left(q_{-}\right), s_{-}\right) \ni s \rightarrow\left(s, q_{-}\right)$extends $\lambda$ which is not possible; thus $s_{-}=t_{-}\left(q_{-}\right)$. By compactness of $\Sigma_{0, C}$ there exists $\epsilon>0$ such

\footnotetext{
${ }^{8}$ The reader should be warned about a slight clash of notation here, due to the fact that we have run out of reasonable symbols for denoting hypersurfaces: $\Sigma_{1}$ in this Lemma is not the asymptotic end of $\Sigma$, as defined in Definition 2.1
} 
that $\left.\left(u-t_{-}\right)\right|_{\Sigma_{0, C}} \geq \epsilon$. We thus have $\lim _{s_{i} \rightarrow s_{-}} u\left(\gamma\left(s_{i}\right)\right)=u\left(q_{-}\right) \geq \epsilon+t_{-}\left(q_{-}\right)=\epsilon+s_{-}$. Set $f(s)=s-u(\gamma(s))$; continuity of $u(\gamma(s))$ implies that there exists $s_{1} \in\left(s_{-}, s_{+}\right)$such that $\left.f\right|_{\left(s_{-}, s_{1}\right)}<0$. One shows similarly that there exists $s_{2} \in\left(s_{-}, s_{+}\right)$such that $\left.f\right|_{\left(s_{2}, s_{+}\right)}>0$, by continuity of $f$ there exists $s_{0} \in\left(s_{-}, s_{+}\right)$such that $f\left(s_{0}\right)=0$, so $\left(s_{0}, \gamma\left(s_{0}\right)\right) \in \Sigma_{1} \cap \lambda \neq \emptyset$, which is what had to be established.

Proof of Theorem 4.1: Let $\tau$ be the time function as given by Proposition 4.1; we want to show that Bartnik's interior condition ([2] [eq. (5.4)] or [5] [eq. (4.4)]) holds. Set

$$
M_{\delta}=\bigcup_{t \in(-\infty, \infty)} \phi[X]_{t}\left(\Sigma_{0, \delta}\right)
$$

where $\Sigma_{0, \delta} \equiv \Sigma_{0, \delta}(\tau)$, with $\Sigma_{0, \delta}(\tau)$ defined by (4.6). Let $q \in \partial M_{\delta}, p \in M_{\delta} \backslash I(q)$, where $I(q)$ denotes the set of points causally related to $q$. By Corollary 3.4 there exists $t_{0}$ such that $\phi[X]_{t_{0}}(q) \in \Sigma_{0}(\tau)$. We have

$$
\begin{aligned}
|\tau(p)-\tau(q)|=\left|\tau\left(\phi[X]_{t_{0}}(p)\right)\right| & \leq \sup _{p^{\prime} \in M_{\delta} \backslash I\left(\phi[X]_{t_{0}}(q)\right)}\left|\tau\left(p^{\prime}\right)\right| \\
& \leq \sup _{q^{\prime} \in \partial \Sigma_{0, \delta}, p^{\prime} \in M_{\delta} \backslash I\left(q^{\prime}\right)}\left|\tau\left(p^{\prime}\right)\right| .
\end{aligned}
$$

For $q^{\prime} \in \partial \Sigma_{0, \delta}$ let $\mathcal{N}=\partial\left(M_{\delta} \backslash I\left(q^{\prime}\right)\right)$; since $\mathcal{N}$ is null, uniform boundedness of light-cone slopes in $M_{\text {ext }}$ implies the existence of a constant $C=C(\delta)$ such that we have

$$
\left|\tau_{\mid \mathcal{N}}\right| \leq C
$$

It follows that $M_{\delta} \backslash I\left(q^{\prime}\right) \subset \tilde{\mathcal{D}}\left(\bigcup_{|t| \leq C} \Sigma_{t, \delta}\right)$ which by Corollary 3.5 is a compact set, and therefore there exists a constant $C_{1}$ such that

$$
|\tau(p)-\tau(q)| \leq C_{1},
$$

which had to be established. Existence of a maximal slice $\hat{\Sigma}_{0}$ follows by the arguments of Proposition 4.3 and Theorem 4.1 of [5]. $\hat{\Sigma}_{0}$ is an asymptotically flat graph by construction and thus a Cauchy surface by Lemma 4.2. The hypersurfaces $\hat{\Sigma}_{t}=\phi[X]_{t}\left(\hat{\Sigma}_{0}\right)$ cover $\stackrel{o}{\mathcal{D}}(\Sigma)$ by Corollary 3.4. If moreover the timelike convergence condition holds, the family $\left\{\hat{\Sigma}_{t}\right\}$ forms a foliation of $\stackrel{o}{\mathcal{D}}(\Sigma)$ (cf. e.g. the arguments of the proof of Theorem 5.4 of [0], and [3] [Section 5]) which completes the proof of Theorem 4.1.

Proof of Theorem 4.2: Let $\tilde{\tau}$ be the time function given by Proposition 4.2. By Theorem 3.1 of [5] $\Sigma_{0}(\tilde{\tau})$ can be deformed outside of a compact set $K \subset \Sigma_{0}(\tilde{\tau}), \partial K \supset S$, to an asymptotically flat hypersurface $\widetilde{\Sigma}$ the mean extrinsic curvature of which is compactly supported. By construction, $\widetilde{\Sigma}$ is an asymptotically flat $\tilde{\tau}$-graph of a function $\tilde{u}$, with $\left.\tilde{u}\right|_{K}=0$, so that $\widetilde{\Sigma}$ is a Cauchy surface for $\stackrel{o}{\mathcal{D}}(\Sigma)$ by Lemma 4.2 . Enlarging $K$ if necessary the function $\tilde{\tau}_{1}=\tilde{\tau}-\tilde{u}$ is a time function on a neighbourhood $\mathcal{U}$ as given by Proposition 4.2, and $\tilde{\tau}_{1 \mid M_{\text {ext }}}$ is the Killing time based on $\widetilde{\Sigma}$, as described in Proposition 2.1. Let $\rho$ be such that the hypersurfaces $\widetilde{\Sigma}_{t}$ are maximal outside of $\widetilde{\Sigma}_{t, \rho}$, set $\widetilde{\Sigma}_{\text {ext }}=\widetilde{\Sigma} \backslash \widetilde{\Sigma}_{0, \rho}$, define

$$
\partial \widetilde{M}_{\sigma}=\bigcup_{t \in[-\sigma, \sigma]} \partial \widetilde{\Sigma}_{t, \rho}
$$


Because the light cones in $\widetilde{M}_{\text {ext }}=\bigcup_{t \in(-\infty, \infty)} \widetilde{\Sigma}_{t \text {,ext }}$ have uniformly bounded slopes it follows that there exists a constant $K_{1}$ such that if $\Sigma$ is a spacelike hypersurface then we have the implication

$$
\Sigma \cap \partial \widetilde{M}_{1} \neq \emptyset \Longrightarrow \Sigma \cap \partial \widetilde{M}_{\mathrm{ext}} \subset \partial \widetilde{M}_{K_{1}}
$$

By Theorem 4.2 of [2] and by Theorem 3.2 for $i \in \mathbb{N}$ there exists a maximal surface $\Sigma_{i}$ such that $\partial \Sigma_{i}=\partial \widetilde{\Sigma}_{0, i}$.

For a set $\Omega$ define

$$
\begin{aligned}
& t_{+}(\Omega)=\sup \left\{\tilde{\tau}_{1}(p): p \in \Omega \cap \partial M_{\text {ext }}\right\} \\
& t_{-}(\Omega)=\inf \left\{\tilde{\tau}_{1}(p): p \in \Omega \cap \partial M_{\text {ext }}\right\} .
\end{aligned}
$$

We have

$$
t_{ \pm}\left(\phi[X]_{t}(\Omega)\right)=t_{ \pm}(\Omega)+t
$$

thus there exists a constant $t_{i}$ such that

$$
t_{-}\left(\phi[X]_{t_{i}}\left(\Sigma_{i}\right)\right)=1 .
$$

Set $\phi[X]_{i} \equiv \phi[X]_{t_{i}}$. (4.23) and (4.22) imply

$$
\phi[X]_{i}\left(\Sigma_{i}\right) \cap \partial \widetilde{M}_{\text {ext }} \subset \partial \widetilde{M}_{K_{1}},
$$

and spacelikeness of $\Sigma_{i}$ gives

$$
\phi[X]_{i}\left(\Sigma_{i}\right) \cap \widetilde{M}_{\text {int }} \subset K=\tilde{\mathcal{D}}\left(\bigcup_{t \in\left[-K_{1}, K_{1}\right]} \phi[X]_{t}\left(\widetilde{\Sigma}_{\text {int }}\right)\right),
$$

where $\widetilde{M}_{\text {int }}=M \backslash \widetilde{M}_{\text {ext }} . K$ is compact by Corollary 3.6, thus

$$
\left.\left|\tilde{\tau}_{1}\right|\right|_{\phi[X]_{i}\left(\Sigma_{i}\right) \cap \widetilde{M}_{\text {int }}} \leq C=\sup _{p \in K}\left|\tilde{\tau}_{1}(p)\right| .
$$

Suppose first that $t_{i} \leq 0$. It follows that $\left.\tilde{\tau}_{1}\right|_{\partial \phi[X]_{i}\left(\Sigma_{i}\right)} \leq 0$, and we conclude from the estimate (3.14) of [2] [Theorem 3.1] that there exists a constant $C$ such that the "tilt function" $\nu_{i}=$ $\nu\left(\phi[X]_{i}\left(\Sigma_{i}\right)\right)(c f$. [2] for details) satisfies

$$
\left|\nu_{i}\right|_{\phi[X]_{i}\left(\Sigma_{i}\right) \cap \partial \widetilde{M}_{\mathrm{ext}}} \leq C
$$

Thus the argument of the proof of Theorem 5.3 of [2] applies to give

$$
t_{i} \geq-C_{1}
$$

for some constant $C_{1}$. Suppose now that $t_{i} \geq K_{1}+1$. It follows from (4.22)-(4.23) that there exists $0>s_{i} \geq-K_{1}-1$ such that

$$
t_{+}\left(\phi[X]_{t_{i}+s_{i}}\left(\Sigma_{i}\right)\right)=-1
$$

and since $t_{i}+s_{i} \geq 0$ one can again conclude as above, from Theorems 3.1 and 5.3 of [2], that

$$
t_{i}+s_{i} \leq C_{2} \Longrightarrow t_{i} \leq C_{2}+K_{1}+1
$$


(4.26) together with (4.24) gives

$$
\left|t_{i}\right| \leq C_{3}
$$

for some constant $C_{3}$, and from what has been said it follows that

$$
\left.\left|\tilde{\tau}_{1}\right|\right|_{\Sigma_{i}} \leq C_{4}
$$

From the estimate (3.10) of [2] we conclude that

$$
\left|\nu\left(\Sigma_{i}\right)\right| \leq C_{5}
$$

and a standard method, together with barrier considerations using the barriers $u_{ \pm}= \pm C r^{-\alpha}$ if $\alpha \in(0,1), u_{ \pm}= \pm C r^{-1} \log r$ if $\alpha=1$ (cf. e.g. Lemma 2.2 of [5]) shows that there exists a subsequence converging to an asymptotically flat maximal surface $\hat{\Sigma}_{0}$. The hypersurfaces $\hat{\Sigma}_{t}$ are obtained by setting $\hat{\Sigma}_{t}=\phi[X]_{t}\left(\hat{\Sigma}_{0}\right)$. By Corollary 3.4 for $p \in \stackrel{o}{\mathcal{D}}(\Sigma)$ every orbit $\phi[X]_{t}(p)$ intersects $\hat{\Sigma}_{0}$, so that the hypersurfaces $\left\{\hat{\Sigma}_{t}\right\}_{t \in \mathbb{R}}$ cover $\stackrel{o}{\mathcal{D}}(\Sigma)$. If the timelike convergence condition holds, then the hypersurfaces $\hat{\Sigma}_{t} \backslash S$ foliate $\stackrel{o}{\mathcal{D}}(\Sigma)$ and the proof is completed.

\section{A A generalization — stationary-rotating space-times.}

It is an interesting feature of the causal structure theory presented in Section 3 that several results follow just from the fact that the orbits of the isometry group are time-oriented "near spacelike infinity", so that timelikeness of the Killing vector is never used. This leads to the following curious generalization of our results to space-times which are asymptotically "stationary-rotating", in the following sense:

Definition A.1 A spacetime $\left(M, g_{a b}\right)$ possessing an acausal slice $\Sigma$ expressed in the form $\Sigma=\Sigma_{1} \cup \Sigma^{\prime}$ will be called $(k, \alpha)$-asymptotically stationary-rotating with respect to the "end" $\Sigma_{1}$ if conditions 1 and G of Definition 2.1 hold, moreover:

3. On $M$ there exists a Killing vector field $X$ the orbits of which are complete. Furthermore, in the coordinate system of point 1 of Definition 2.1 we have as $r \rightarrow \infty$,

$$
X=\left(A+O\left(r^{-\alpha}\right)\right) \frac{\partial}{\partial t}+\left(\omega_{j}^{i} x^{j}+O\left(r^{-\alpha}\right)\right) \frac{\partial}{\partial x^{i}}
$$

for some constant $A(\neq 0)$, and some constant coefficients matrix $\omega_{i}^{j}$ satisfying $\omega_{i}^{j}=-\omega_{j}^{i}$. We moreover assume that the vector field

$$
Y=X-\hat{X}
$$

is uniformly timelike on $\Sigma_{1}\left(g_{\mu \nu} Y^{\mu} Y^{\nu}<-\epsilon\right.$ for some constant $\left.\epsilon>0\right)$, where

$$
\hat{X}^{\mu} \partial_{\mu}=\omega_{i}^{j} x^{i} \partial_{j}
$$

If $\omega_{i}^{j} \neq 0$ we shall moreover require that the metric satisfies

$$
\forall \quad 0 \leq|\beta|+i \leq k \quad \partial_{x}^{\beta} \mathcal{L}_{\hat{X}}^{i}\left(g_{\mu \nu}-\eta_{\mu \nu}\right)=O\left(r^{-\alpha-|\beta|-i}\right),
$$




$$
\begin{aligned}
& \forall \quad|\beta|+i=k,|x-y| \leq r(x) / 2 \quad\left|\partial_{x}^{\beta} \mathcal{L}_{\hat{X}}^{i} g_{\mu \nu}\left(0, x^{k}\right)-\partial_{x}^{\beta} \mathcal{L}_{\hat{X}}^{i} g_{\mu \nu}\left(0, y^{k}\right)\right| \leq C r^{-\alpha-k-\lambda}|x-y|^{\lambda}, \\
& \forall \quad|\beta|+i=k, 0 \leq|s| \leq 2 \pi A|\omega|^{-1} \quad\left|\partial_{x}^{\beta} \mathcal{L}_{\hat{X}}^{i}\left[(R(s)-\mathrm{id})^{*} g_{\mu \nu}\right]\left(0, x^{i}\right)\right| \leq C r^{-\alpha-k-\lambda}|s|^{\lambda},
\end{aligned}
$$

where $\beta$ is a multi-index with space indices only, $R_{i}^{j}(s)=\exp \left\{s \omega_{i}^{j}\right\},(R(s)-\mathrm{id})^{*}$ is the pull-back by the map $R_{i}^{j}(s) x^{i}-x^{j}$ and $\mathcal{L}_{\hat{X}}$ is the Lie derivative. We shall also assume, for $\omega_{i}^{j} \neq 0$, that the vector field $Y$ satisfies

$$
\begin{gathered}
\forall \quad 0 \leq|\beta|+i \leq k \quad \partial_{x}^{\beta} \mathcal{L}_{\hat{X}}^{i} Y_{\mu}=O\left(r^{-\alpha-|\beta|-i}\right), \\
\forall \quad|\beta|+i=k,|x-y| \leq r(x) / 2 \quad\left|\partial_{x}^{\beta} \mathcal{L}_{\hat{X}}^{i} Y_{\mu}\left(0, x^{k}\right)-\partial_{x}^{\beta} \mathcal{L}_{\hat{X}}^{i} Y_{\mu}\left(0, y^{k}\right)\right| \leq C r^{-\alpha-k-\lambda}|x-y|^{\lambda}, \\
\forall \quad|\beta|+i=k, 0 \leq|s| \leq 2 \pi A|\omega|^{-1} \quad\left|\partial_{x}^{\beta} \mathcal{L}_{\hat{X}}^{i}\left[(R(s)-\mathrm{id})^{*} Y_{\mu}\right]\left(0, x^{i}\right)\right| \leq C r^{-\alpha-k-\lambda}|s|^{\lambda}
\end{gathered}
$$

\section{Remarks:}

1. If $|\omega|=0$, then Definition A.1 clearly reduces to Definition 2.1.

2. If $\hat{X}=\omega_{j}^{i} x^{j} \partial_{i}$ is also a Killing vector field, such that $[X, \hat{X}]=0$, as occurs e.g. in the case of the Kerr space-time, then (A.2)-(A.7) will clearly hold if (2.1)-(2.4) are fulfilled.

3. If ( $\mathrm{A.2})$ and (A.5) hold with $1 \leq i \leq k+1$, then (A.3) $-($ A.4 $)$ and (A.6) $-($ A.7) will hold as well (with any $0 \leq \lambda \leq 1$ ).

Proposition A.1 (Killing time based on $\left.\Sigma \cap M_{1}\right)$ Let $\left(M, g_{a b}\right)$ with $\Sigma=\Sigma_{1} \cup \Sigma^{\prime}$ be asymptotically stationary with respect to $\Sigma_{1}$. Then there exists a global coordinate system on $M_{1}$ such that $M_{1} \approx \mathbb{R} \times\left(\mathbb{R}^{3} \backslash B\left(R_{1}\right)\right), \Sigma_{1} \subset\{t=0\}$, and

$$
\begin{gathered}
g_{00}<-C^{-1}, \quad A \frac{\partial g_{\mu \nu}}{\partial t}+\omega_{j}^{i} x^{j} \frac{\partial g_{\mu \nu}}{\partial x^{i}}=0 \\
\left(\Longrightarrow \quad\left\{\begin{array}{ll}
g_{\mu \nu}\left(t+s, x^{i}\right)=g_{\mu \nu}\left(t, x^{i}\right), s \in \mathbb{R} & \text { if }|\omega|=0, \\
g_{\mu \nu}\left(t+2 \pi A|\omega|^{-1}, x^{i}\right)=g_{\mu \nu}\left(t, x^{i}\right), & \text { otherwise, }
\end{array}\right)\right. \\
\forall Z^{i} \in \mathbb{R}^{3} \quad g_{i j} Z^{i} Z^{j} \geq C^{-1} \sum_{i=1}^{3}\left(Z^{i}\right)^{2} \\
\left|\partial_{\sigma_{1}} \ldots \partial_{\sigma_{i}}\left(g_{\mu \nu}-\eta_{\mu \nu}\right)\right| \leq C r^{-\alpha-i} \quad 0 \leq i \leq k
\end{gathered}
$$

for some constant $C$, with $|\omega|=\sum_{i<j} \sqrt{\left(\omega_{j}^{i}\right)^{2}} ;$ moreover, $\partial_{\sigma_{1}} \ldots \partial_{\sigma_{k}} g_{\mu \nu}$ satisfy an obvious weighted Hölder condition. 
Proof: Let $x^{\mu}$ be the coordinates of point 1 of definition 2.1, let $\phi[X]^{\mu}\left(s, y^{i}\right)$ be the unique solution of the problem

$$
\left\{\begin{array}{l}
\frac{d \phi[X]^{\mu}}{d s}\left(s, y^{i}\right)=X^{\mu}\left(\phi[X]^{\mu}\left(s, y^{i}\right)\right), \\
\phi[X]^{0}\left(0, y^{i}\right)=0, \quad \phi[X]^{j}\left(0, x^{i}\right)=x^{j} .
\end{array}\right.
$$

Let the new coordinates $\left(y^{\mu}\left(x^{\alpha}\right)\right)=\left(A s\left(x^{\alpha}\right), y^{i}\left(x^{\alpha}\right)\right)$ be implicitly defined by the equations

$$
\begin{aligned}
& x^{o}=\phi[X]^{0}\left(s, y^{i}\right), \\
& x^{i}=\phi[X]^{j}\left(s, y^{k}\right) .
\end{aligned}
$$

Since the derivatives of $X$ satisfy uniform decay conditions up to order $k$, it follows from (A.11) by standard ODE theory that the derivatives of $\phi[X]^{\mu}$ will satisfy uniform decay conditions up to order $k$, then by (A.12) the derivatives of $\partial x^{\mu} / \partial y^{\nu}$ will satisfy uniform decay conditions up to order $k-1$; it turns out, however, that decay conditions for the derivatives of the metric hold at order $k$ as well, which can be seen as follows: differentiating equation (A.12) with respect to $x^{\mu}$ one obtains:

$$
\begin{gathered}
1=\frac{\partial x^{0}}{\partial x^{0}}=X^{0} \frac{\partial s}{\partial t}+\frac{\partial \phi[X]^{0}}{\partial y^{i}} \frac{\partial y^{i}}{\partial t} \\
0=\frac{\partial x^{0}}{\partial x^{i}}=X^{0} \frac{\partial s}{\partial x^{i}}+\frac{\partial \phi[X]^{0}}{\partial y^{j}} \frac{\partial y^{j}}{\partial x^{i}}, \\
0=\frac{\partial x^{i}}{\partial t}=X^{j} \frac{\partial s}{\partial t}+\frac{\partial \phi[X]^{j}}{\partial y^{k}} \frac{\partial y^{k}}{\partial t}, \\
\delta_{j}^{i}=X^{\ell} \frac{\partial s}{\partial x^{j}}+\frac{\partial \phi[X]^{\ell}}{\partial y^{k}} \frac{\partial y^{k}}{\partial x^{j}} .
\end{gathered}
$$

From (A.11) and (A.13) at $s=0$ it follows that

$$
\begin{gathered}
\left.\frac{\partial s}{\partial t}\right|_{s=0}=\left.\left(X^{0}\right)^{-1}\right|_{s=0},\left.\quad \frac{\partial s}{\partial x^{i}}\right|_{s=0}=0, \\
\left.\frac{\partial y^{i}}{\partial t}\right|_{s=0}=-\left.\left(X^{0}\right)^{-1} X^{i}\right|_{s=0},\left.\quad \frac{\partial y^{i}}{\partial x^{j}}\right|_{s=0}=\delta_{j}^{i} .
\end{gathered}
$$

To avoid ambiguities let us write the metric in the form $g=g_{x^{\mu} x^{\nu}} d x^{\mu} d x^{\nu}=g_{y^{\mu}} y^{\nu} d y^{\mu} d y^{\nu}$; we have $X=X^{x^{0}} \partial / \partial t+X^{x^{i}} \partial / \partial x^{i}=X^{y^{0}} \partial / \partial y^{0}+X^{y^{i}} \partial / \partial y^{i}=A \partial / \partial y^{0}$, and from (A.14) one obtains

$$
\begin{aligned}
& g_{y^{i} y^{j}}\left(y^{0}, y^{i}\right)=g_{x^{i} x^{j}}\left(x^{0}=0, x^{i}=y^{i}\right), \\
& g_{y^{0} y^{i}}\left(y^{0}, y^{i}\right)=A^{-1}\left[g_{x^{i} x^{\ell}} X^{x^{\ell}}+g_{x^{i} x^{0}} X^{x^{0}}\right]\left(x^{0}=0, x^{i}=y^{i}\right), \\
& g_{y^{0} y^{0}}\left(y^{0}, y^{i}\right)=A^{-2}\left[g_{x^{0} x^{0}}\left(X^{x^{0}}\right)^{2}+g_{x^{k} x^{\ell}} X^{x^{k}} X^{x^{\ell}}+2 g_{x^{k} x^{0}} X^{x^{0}} X^{x^{k}}\right]\left(x^{0}=0, x^{i}=y^{i}\right),
\end{aligned}
$$

and for $\omega=0$ the asymptotic bounds readily follow from (2.1)-(2.5) and (2.8). If $\omega \neq 0$ the coordinates $\left\{y^{\mu}\right\}$ are, however, not asymptotically flat. This is easily cured as follows: let 
$R_{i}^{j}(s) \equiv \exp \left\{s \omega_{i}^{j}\right\}$; thus $R_{i}^{j}(s)$ is a rotation by angle $|\omega| s$ around the appropriately oriented axis $\hat{e}^{i}$ defined by $\omega_{i}^{j} \hat{e}^{i}=0$, where $|\omega|=\sqrt{\sum_{i<j}\left(\omega_{i}^{j}\right)^{2}}$, and

$$
\frac{d R_{i}^{j}(s)}{d s}=R_{i}^{k}(s) \omega_{k}^{j}=\omega_{i}^{k} R_{k}^{j}(s) .
$$

Let new coordinates $\left\{z^{\mu}\right\}$ be defined by

$$
z^{0}=y^{0}, \quad z^{i}=R_{k}^{i}(s) y^{k}
$$

one easily finds

$$
\begin{gathered}
g_{z^{i} z^{j}}\left(z^{0}, z^{i}\right)=g_{x^{k} x^{\ell}}\left(x^{0}=0, x^{i}=R_{j}^{i}\left(-A^{-1} z^{0}\right) z^{j}\right) R_{i}^{k}\left(-A^{-1} z^{0}\right) R_{j}^{\ell}\left(-A^{-1} z^{0}\right), \\
g_{z^{0} z^{i}}\left(z^{0}, z^{i}\right)=A^{-1}\left[g_{x^{k} x^{\ell}}\left(X^{x^{\ell}}-\omega_{j}^{\ell} x^{j}\right)+g_{x^{k} x^{0}} X^{x^{0}}\right]\left(x^{0}=0, x^{i}=R_{j}^{i}\left(-A^{-1} z^{0}\right) z^{j}\right) R_{i}^{k}\left(-A^{-1} z^{0}\right), \\
g_{z^{0} z^{0}}\left(z^{0}, z^{i}\right)=A^{-2}\left[g_{x^{0} x^{0}}\left(X^{x^{0}}\right)^{2}+g_{x^{k} x^{\ell}}\left(X^{x^{k}}-\omega_{j}^{k} x^{j}\right)\left(X^{x^{\ell}}-\omega_{m}^{\ell} x^{m}\right)\right. \\
\left.+2 g_{x^{k} x^{0}} X^{x^{0}}\left(X^{x^{k}}-\omega_{j}^{k} x^{j}\right)\right]\left(x^{0}=0, x^{i}=R_{j}^{i}\left(-A^{-1} z^{0}\right) z^{j}\right) .
\end{gathered}
$$

We have, e.g.,

$$
\partial_{z_{0}} g_{z^{i} z^{j}}=O\left(r^{-\alpha-1}\right) \quad \Longleftrightarrow \quad \mathcal{L}_{\hat{X}} g_{x^{k} x^{\ell}}=O\left(r^{-\alpha-1}\right),
$$

with $\hat{X}=\omega_{j}^{i} x^{j} \partial_{i}$, and (A.8)-(A.10) follow in a similar manner from (A.2)-(A.7).

Remark: Equations (A.15)-A.17) provide a very effective way of constructing stationary metrics of the form (1.1) with some "desirable" pathological properties (not necessarily, however, satisfying some reasonable field equations and/or energy inequalities): fix a three dimensional manifold $\Sigma$, with a metric $g_{x^{i} x^{j}} d x^{i} d x^{j}$, a scalar field $g_{x^{0} x^{0}}$, a one form $g_{x^{i} x^{0}} d x^{i}$, a vector field $X^{i} \partial / \partial x^{i}$, and finally a scalar field $X^{0}>0$; make sure that the matrix $g_{x^{\mu} x^{\nu}}$ has Lorentzian signature. Then the manifold $\mathbb{R} \times \Sigma$ with the metric $g_{y^{\mu}} y^{\nu} d y^{\mu} d y^{\nu}$, with $g_{y^{\mu}} y^{\nu}$ given by (A.15)-(A.17), is a Lorentzian manifold with Killing vector $X^{\mu} \partial / \partial x^{\mu}=\partial / \partial y^{0}$. As an illustration, consider $\Sigma=\mathbb{R}^{3}$ with $g_{x^{\mu} x^{\nu}}\left(x^{i}\right) d x^{\mu} d x^{\nu}=-\left(d x^{0}\right)^{2}+\sum\left(d x^{i}\right)^{2}, X^{\mu}\left(x^{i}\right) \partial / \partial x^{\mu}=$ $\phi\left(x^{i}\right) \partial / \partial x^{0}+\partial / \partial x^{1}$, where $\phi$ is any smooth radially symmetric function satisfying

$$
\phi \begin{cases}=2, & r \leq 10^{3} \\ \in\left[1+10^{-400}, 2\right], & 10^{3} \leq r \leq 10^{4} \\ =1+r^{-100}, & r \geq 10^{4}\end{cases}
$$

Then $X^{\mu} \partial / \partial x^{\mu}$ is timelike everywhere, asymptotically approaches the null vector $\partial / \partial x^{0}+$ $\partial / \partial x^{1}$, and we have $g_{y^{\mu} y^{\nu}} d y^{\mu} d y^{\nu}=\left(1-\phi^{2}\right)\left(d y^{0}\right)^{2}+2 d y^{0} d y^{1}+\sum\left(d y^{i}\right)^{2}$. Introducing $z^{0}=y^{0}$, $z^{1}=y^{1}+y^{0}, z^{A}=y^{A}, A=1,2$, one has $g_{z^{\mu} z^{\nu}} d z^{\mu} d z^{\nu}=-\tilde{\phi}^{2}\left(d z^{0}\right)^{2}+\sum\left(d z^{i}\right)^{2}$, with $\tilde{\phi}\left(z^{i}\right)=$ $\phi\left(z^{1}-z^{0}, z^{2}, z^{3}\right)$, thus all the conditions of Definition 2.1 are satisfied (with any $2 \leq k \leq 98$, $\alpha=99-k$ and $\lambda=1$ ) except for uniform timelikeness of $X$ and for equation (2.5). Moreover it is clear that no coordinates as in Proposition 2.1 exist for this metric.

We have the following

Theorem A.1 All the results of this paper hold with stationary replaced by stationary-rotating. 
The reader who wishes to check the validity of this result will notice that essentially all the proofs have been worded in a way which generalizes immediately to the stationary-rotating case. Let us mention the following. First, it is easily seen from Proposition A.1 that for any $p=(t, x) \in M_{\text {ext }}$ the orbits $\gamma_{p}(t)$ are future or past oriented, according to the sign of $A$ (the sequence $t_{i}$ can be taken to be equal to $\left.2 \pi i A|\omega|^{-1}, i \in \mathbf{Z}\right)$.

A property of the orbits of $\phi[X]$ which is often used is the following: let $p, q \in M_{i}$, then there exists $T$ such that, changing the time orientation if necessary for all $t \leq T$ we have $\phi[X]_{t}(p) \in$ $I^{-}(q)$. This can be seen as follows: joining points with curves of the form $(t+B s, r+s)$ and $(t+B s, \gamma(s))$, where $t, r$ refer to the coordinates of Proposition A.1, $B$ is a constant large enough, and $\gamma(s)$ is either a constant curve or, say, a geodesic arc in the standard round metric on the spheres $r=$ const, one finds that there exists a constant $C=C(p, q)$ such that all points $p^{\prime}=\left(t\left(p^{\prime}\right), x\left(p^{\prime}\right)\right)$ with $t\left(p^{\prime}\right) \geq \max (t(p), t(q))+C, r_{0}=\min (r(p), r(q)) \leq r\left(p^{\prime}\right) \leq$ $\max (r(p), r(q))$ satisfy $p^{\prime} \in I^{+}(p)$, and $p^{\prime} \in I^{+}(q)$; similarly if $t\left(p^{\prime}\right) \leq \min (t(p), t(q))-C$ and $r_{0}=\min (r(p), r(q)) \leq r\left(p^{\prime}\right) \leq \max (r(p), r(q))$, then $p^{\prime} \in I^{-}(p)$, and $p^{\prime} \in I^{-}(q)$. In the Killing coordinates on $M_{i}$ we have $\phi[X]_{s}(t, x)=(t+A s, R(s) x)$, where $R(s)$ is a rotation (around an appropriate axis, $c f$. the proof of Proposition A.1) by an angle $|\omega| s$, and setting $T=-A^{-1}(\max (|t(p)|,|t(q)|)+C)$ the result follows.

\section{B An Alternate Proof of Existence of a Maximal Foliation for Spacetimes of Class (b) Satisfying the Strong Energy Condition}

In this Appendix we shall outline a somewhat simpler though certainly less elegant proof of the Theorems of existence of maximal slices for space-times of class (b) when one assumes that the timelike convergence condition (4.3) holds:

Theorem B.1 Let $(M, g)$ be a space-time of class (b), suppose that (4.1) holds and that the timelike convergence condition (4.3) is satisfied. Then $\stackrel{o}{\mathcal{D}}(\Sigma)$ can be covered by a family of maximal (spacelike) asymptotically flat hypersurfaces $\Sigma_{s}, s \in \mathbb{R}$, with boundary $\partial \Sigma_{s}=\partial \Sigma=$ $S$, such that

$$
\phi[X]_{t}\left(\Sigma_{s}\right)=\Sigma_{s+t}, \quad \text { and } \quad \Sigma_{s} \approx \Sigma .
$$

Moreover $\Sigma_{s} \backslash S$ are Cauchy surfaces for $\mathcal{D}(\Sigma)$, and the hypersurfaces $\Sigma_{s} \backslash S$ foliate $\stackrel{o}{\mathcal{D}}(\Sigma)$.

Proof: The idea of the proof is similar to that of the proof of Theorem 4.2, the essential difference being how the appropriate time function is constructed. By [5] one can deform $\Sigma$ outside of a compact set so that, still denoting the deformed hypersurface by $\Sigma, \Sigma \backslash \Sigma_{0, \rho}$ is maximal. By Theorem 4.1 of [3] there exists a maximal surface $\check{\Sigma} \approx \Sigma_{0, \rho}$ such that $\partial \check{\Sigma}=$ $S \cup \partial \Sigma_{0, \rho}$. By the gluing Lemma B.1 which is proved below the hypersurface $\check{\Sigma} \cup\left(\Sigma \backslash \Sigma_{0, \rho}\right)$ can be smoothed out to a smooth hypersurface $\hat{\Sigma}$; by construction there exists $\epsilon$ such $\hat{\Sigma}_{0, \rho-\epsilon}$ and $\hat{\Sigma} \backslash \hat{\Sigma}_{0, \rho+\epsilon}$ are maximal. We can choose $\epsilon$ small enough so that $\partial \Sigma_{0, \rho \pm \epsilon}$ are smooth coordinate spheres in $M_{\text {ext }}$. Because the slopes of the light cones in $M_{\text {ext }}$ are uniformly bounded, there exists a constant $K_{1}$ such that for any spacelike surface $\tilde{\Sigma}$ we have the implication

$$
\tilde{\Sigma} \cap \mathcal{T}_{1} \neq \emptyset \Longrightarrow \tilde{\Sigma} \cap \mathcal{T}_{\infty} \subset \mathcal{T}_{K_{1}}
$$


where

$$
\mathcal{T}_{s} \equiv \cup_{t \in(-s, s)} \phi[X]_{t}\left(\hat{\Sigma}_{0, \rho+\epsilon} \backslash \hat{\Sigma}_{0, \rho-\epsilon}\right) .
$$

Let $\Sigma_{i}$ be a maximal surface such that $\partial \Sigma_{i}=S \cup \partial \hat{\Sigma}_{0, i}$. Let $s$ be such that $\phi[X]_{s}\left(\Sigma_{i}\right) \cap \mathcal{T}_{1} \neq \emptyset$. We then have $\phi[X]_{s}\left(\Sigma_{i}\right) \cap \mathcal{T}_{\infty} \subset \mathcal{T}_{K_{1}}$. Set

$$
M_{\text {int }} \equiv M \backslash\left[\cup_{t \in \mathbb{R}}\left(\hat{\Sigma}_{t} \backslash \hat{\Sigma}_{t, \rho-\epsilon}\right)\right] .
$$

It follows that $\phi[X]_{s}\left(\Sigma_{i}\right) \cap M_{\text {int }}$ is a maximal surface with boundary included in $S \cup_{t \in\left[-K_{1}, K_{1}\right]}$ $\partial \hat{\Sigma}_{t, \rho-\epsilon}$. Because $\phi[X]_{ \pm K_{1}}\left(\hat{\Sigma}_{0, \rho-\epsilon}\right)$ are maximal, the comparison principle implies that $\phi[X]_{s}\left(\Sigma_{i}\right) \cap$ $M_{\text {int }}$ is a subset of the compact set $\cup_{t \in\left[-K_{1}, K_{1}\right]} \hat{\Sigma}_{t, \rho-\epsilon}$. This observation allows one to carry out the remaining arguments of the proof as in Theorem 4.2, by using any time function which coincides with the Killing time in $M_{\text {ext }}$, and covers a neighbourhood of $\cup_{t \in\left[-K_{1}-1, K_{1}+1\right]} \hat{\Sigma}_{t, \rho+\epsilon}$. (Such a time function can be easily constructed spanning maximal surfaces on some timelike hypersurface containing $\partial \Sigma$ and on $\cup_{t \in\left[-K_{1}-1, K_{1}+1\right]} \partial \hat{\Sigma}_{t, \rho+\epsilon}$, and appropriately "rounding off" any nondifferentiability if necessary.)

Let us finally prove the "gluing Lemma":

Lemma B.1 Let $\Sigma$ be a $C^{k, \alpha}, k \geq 1,0<\alpha \leq 1$, spacelike embedded hypersurface in a Lorentzian space-time $\left(M, g_{a b}\right)$ with a $C^{k-1, \alpha}$ metric, suppose that $\widetilde{\Sigma}$ is a $C^{k, \alpha}$ spacelike embedded hypersurface in $M$ such that $\partial \widetilde{\Sigma} \subset \Sigma$ is a compact $C^{k, \alpha}$ submanifold of $\Sigma$, and assume that $\Sigma$ is the disjoint union of $\Sigma_{\text {int }}$, $\partial \widetilde{\Sigma}$ and $\Sigma_{\text {ext }}$, with $\partial \Sigma_{\text {int }}=\partial \widetilde{\Sigma}=\partial \Sigma_{\text {ext }}$. Define the (Lipschitz) piecewise $C^{k, \alpha}$ hypersurface $\hat{\Sigma}$ by:

$$
\hat{\Sigma}=\widetilde{\Sigma} \cup \Sigma_{\text {ext }} .
$$

Let $d(p)$ be the distance on $\hat{\Sigma}$ from $p$ to $\partial \widetilde{\Sigma}$. For every $\epsilon>0$ there exists a spacelike embedded $C^{k, \alpha}$ hypersurface $\hat{\Sigma}_{\epsilon}$ - "a smoothed out deformation of $\hat{\Sigma}$ " - such that

$$
p \in \hat{\Sigma}, \quad p \notin \hat{\Sigma}_{\epsilon} \Longrightarrow d(p)<\epsilon ; \quad \text { edge } \hat{\Sigma}_{\epsilon}=\operatorname{edge} \hat{\Sigma} .
$$

(In other words, $\hat{\Sigma}$ coincides with $\hat{\Sigma}_{\epsilon}$ except for points closer than $\epsilon$ to $\partial \widetilde{\Sigma}$.)

Proof: Let $t$ be any time function defined in a neighbourhood $\mathcal{O}$ of $\partial \widetilde{\Sigma}$ such that $\Sigma \cap \mathcal{O}=$ $\{t=0\}$. [Such a function can e.g. be constructed as follows: let $\mathcal{V} \subset \Sigma$ be a conditionally compact neighbourhood of $\partial \widetilde{\Sigma}$, let $\tilde{g}_{a b}$ be any smooth Lorentzian metric in a neighbourhood $\mathcal{W} \subset M$ of $\overline{\mathcal{V}}$ such that $\Sigma \cap \mathcal{W}$ is spacelike with respect to the metric $\tilde{g}_{a b}$, and such that in every tangent space the solid light cones of $\tilde{g}_{a b}$ are proper subsets of the solid light cones of $g_{a b}$. Let $t \in C^{\infty}(\widetilde{\mathcal{D}}(\mathcal{V}))$ be a solution of the problem

$$
\begin{aligned}
& \tilde{g}^{\mu \nu} \tilde{\nabla}_{\mu} \tilde{\nabla}_{\nu} t=0, \\
& \left.t\right|_{\mathcal{V} \cap \Sigma}=0,\left.\quad \tilde{n}^{\mu} \partial_{\mu} t\right|_{\mathcal{V} \cap \Sigma}=1,
\end{aligned}
$$

where $\widetilde{\nabla}_{\mu}$ is the covariant derivative of the metric $\tilde{g}_{a b}, \tilde{n}^{\mu}$ is a vector field transverse to $\mathcal{W} \cap \Sigma$ and $\widetilde{\mathcal{D}}(\mathcal{V})$ is the domain of dependence of $\mathcal{V}$ in the spacetime $\left(\mathcal{W}, \tilde{g}_{a b}\right)$. Embeddedness of $\Sigma$ implies that there exists an open neighbourhood $\mathcal{O}$ of $\partial \widetilde{\Sigma}$ in $\mathcal{W}$ such that $t$ is a time function for the metric $g_{a b}$ on $\mathcal{O}$, and we have $\Sigma \cap \mathcal{O}=\{t=0\}$.] Let $r$ be any $C^{\infty}$ function on $\mathcal{U}=\Sigma \cap \mathcal{O}$ such that $|d r|>0,\left.r\right|_{\partial \widetilde{\Sigma}}=1$. Let $v$ be any coordinates on $\partial \widetilde{\Sigma}$, one can extend $v$ to $\mathcal{U}$ by Lie 
dragging along the integral curves of $\nabla r ; r$ and $v$ can be extended to $\mathcal{O}$ by Lie dragging along $\nabla t$. By compactness of $\partial \widetilde{\Sigma}$ there exists $0<\delta \leq \frac{1}{2}$ such that we have

$$
(t, r, v) \in K_{\delta}=[-\delta, \delta] \times[1-\delta, 1+\delta] \times \partial \widetilde{\Sigma} \subset \mathcal{O}
$$

Since edge $\tilde{\Sigma}=\partial \Sigma \subset \Sigma$, decreasing $\delta$ if necessary one can find a $C^{k, \alpha}$ function $\tilde{u}$ such that $\widetilde{\Sigma} \cap K_{\delta}$ is a graph:

$$
\widetilde{\Sigma} \cap K_{\delta}=\{t=\tilde{u}(r, v), r \in[1-\delta, 1], v \in \partial \widetilde{\Sigma}\} .
$$

It follows that $\hat{\Sigma}$, as defined by $(\overline{\mathrm{B} .1})$, is a graph of the following $C^{0,1}$ function $u$ :

$$
u(r, v)=\left\{\begin{array}{ll}
\tilde{u}(r, v), & r \in[1-\delta, 1] \\
0 & r \in[1,1+\delta]
\end{array} .\right.
$$

By Whitney's extension Lemma (cf. e.g. [12]) there exists a function $\widetilde{\widetilde{u}} \in C^{k, \alpha}([1-\delta, 1+\delta] \times \partial \widetilde{\Sigma})$ such that $\left.\widetilde{\tilde{u}}\right|_{[1-\delta, 1] \times \partial \widetilde{\Sigma}}=\tilde{u}$. Let $\widetilde{\widetilde{\Sigma}}$ be the graph of $\widetilde{\widetilde{u}}$, decreasing $\delta$ if necessary we may assume that $\widetilde{\widetilde{\Sigma}}$ is a spacelike hypersurface in $K_{\delta}$. Let $m$ be the unit (spacelike) vector field proportional to $\frac{\partial}{\partial r}$, define $n$ to be the future oriented timelike, unit vector field, orthogonal to $\frac{\partial}{\partial r}$, of the form $a \frac{\partial}{\partial t}+b \frac{\partial}{\partial r}$; we thus have

$$
\begin{aligned}
\frac{\partial}{\partial t} & =A(t, r, v) n+B(t, r, v) m \quad(|B|<A) \\
\frac{\partial}{\partial r} & =C(t, r, v) m, \\
n^{2} & =-m^{2}=-1, \quad n \cdot m=0 .
\end{aligned}
$$

By compactness of $K_{\delta}$ it follows that there exists $\delta_{1}>0$ such that

$$
C>\delta_{1}, \quad A>\delta_{1}
$$

The vector field $Y=Y^{\mu} \partial_{\mu}=\frac{\partial \widetilde{\widetilde{u}}}{\partial r} \frac{\partial}{\partial t}+\frac{\partial}{\partial r}=A \frac{\partial \widetilde{\widetilde{u}}}{\partial r} n+\left(C+B \frac{\partial \widetilde{\widetilde{u}}}{\partial r}\right) m$ is tangent to $\widetilde{\Sigma}$, so that compactness of $K_{\delta}$ and spacelikeness of $\widetilde{\tilde{\Sigma}}$ imply that there exists $\delta_{2}>0$ such that

$$
C(\widetilde{\widetilde{u}}(r, v), r, v) \geq\left[A(\widetilde{\widetilde{u}}(r, v), r, v)-\operatorname{sgn}\left(\frac{\partial \widetilde{\widetilde{u}}}{\partial r}(r, v)\right) B(\widetilde{\tilde{u}}(r, v), r, v)\right]\left|\frac{\partial \widetilde{\widetilde{u}}}{\partial r}(r, v)\right|+\delta_{2},
$$

where $\operatorname{sgn}(\cdot)$ denotes the sign of $\cdot$ This together with continuity of $A, B$ and $C$ shows that, decreasing $\delta$ if necessary, there exists $\delta_{3}>0$ such that for all $(t, r, v) \in K_{\delta}$ one has

$$
C(t, r, v) \geq\left[A(t, r, v)-\operatorname{sgn}\left(\frac{\partial \widetilde{\tilde{u}}}{\partial r}(r, v)\right) B(t, r, v)\right]\left[\left|\frac{\partial \widetilde{\tilde{u}}}{\partial r}(r, v)\right|+\delta_{3}\right] .
$$

Let $\epsilon$ be as described in the statement of this Lemma, there exists a constant $K>0$ such that for $|r-1| \leq K \epsilon$ one has $d(r, v) \leq \epsilon$, where $d(p)$ is the geodesic distance on $\widetilde{\Sigma} \cup \Sigma_{\text {ext }}$ from $p=(r, v)$ to $\partial \widetilde{\Sigma}$. Without loss of generality we may assume $K \epsilon \leq \delta / 4$. Let $\phi[X]_{\epsilon} \in C^{\infty}(\mathbb{R})$ be any function satisfying $\operatorname{supp} \phi[X]_{\epsilon} \subset\left(1-\frac{K \epsilon}{2}, 1+\frac{K \epsilon}{2}\right), 0 \leq \phi[X]_{\epsilon} \leq 1, \phi[X]_{\epsilon}(x)=1$ for 
$x \in[1-K \epsilon / 4,1+K \epsilon / 4]$. Let $\psi \in C^{\infty}(\mathbb{R})$ satisfy $\operatorname{supp} \psi \in[-1,1], 0 \leq \psi \leq 1, \int_{-\infty}^{\infty} \psi(x) d x=1$. For $0<\nu<K \epsilon / 2$ set $\psi_{\nu}(x)=\psi\left(\frac{x}{\nu}\right)$, define

$$
u_{\nu, \epsilon}(r, v)=\int_{-\infty}^{\infty} \psi_{\nu}(r-s) \phi[X]_{\epsilon}(s) u(s, v) d s+\left(1-\phi[X]_{\epsilon}(r)\right) u(r, v) .
$$

It follows from (B.8) that

1. $u_{\nu, \epsilon} \leq C^{k, \alpha}([1-\delta, 1+\delta] \times \partial \widetilde{\Sigma})$,

2. $u_{\nu, \epsilon}$ converges to $u$ in $C^{0,1}([1-\delta, 1+\delta] \times \partial \widetilde{\Sigma})$ as $\nu \longrightarrow 0$.

3. For $r \notin[1-K \epsilon, 1+K \epsilon]$ we have $u_{\nu, \epsilon}(r, v)=u(r, v)$; it follows that the hypersurfaces $\Sigma_{\nu, \epsilon}$ defined as those coinciding with $\widetilde{\Sigma}$ or $\Sigma_{\text {ext }}$ outside of $K_{\delta}$, and defined as the graph of $u_{\nu, \epsilon}$ in $K_{\delta}$, are $C^{k, \alpha}$ hypersurfaces.

4. For fixed $r \in[1-\delta, 1+\delta]$ the functions $u_{\nu, \epsilon}(r, \cdot) \in C^{k, \alpha}(\partial \widetilde{\Sigma})$ converge as $\nu \longrightarrow 0$ to $u(r, \cdot)$ in $C^{k, \alpha}(\partial \widetilde{\Sigma})$; in particular there exists $\nu_{0}>0$ such that for $\nu \leq \nu_{0}$ the vector fields $\frac{\partial u_{\nu, \epsilon}}{\partial v^{A}} \frac{\partial}{\partial t}+\frac{\partial}{\partial v^{A}}$ are spacelike.

To show spacelikeness of $\Sigma_{\nu, \epsilon}$ for $\nu$ small enough it thus remains to show that the vector fields $Y_{\nu, \epsilon}=\frac{\partial u_{\nu, \epsilon}}{\partial v} \frac{\partial}{\partial t}+\frac{\partial}{\partial v}$ are spacelike. This is equivalent to the inequality

$$
C\left(u_{\nu, \epsilon}(r, v), r, v\right)>\left[A\left(u_{\nu, \epsilon}(r, v), r, v\right)-\operatorname{sgn}\left(\frac{\partial u_{\nu, \epsilon}}{\partial r}(r, v)\right) B\left(u_{\nu, \epsilon}(r, v), r, v\right)\right]\left|\frac{\partial u_{\nu, \epsilon}}{\partial r}(r, v)\right| .
$$

It follows from $(\overline{B .7}),(\overline{B .9})$ and compactness of $K_{\delta}$ that there exists $\delta_{4}>0$ such that if

$$
\beta(r, v) \equiv\left|\frac{\partial u_{\nu, \epsilon}}{\partial r}(r, v)\right| \leq 2 \delta_{4} \quad \Longrightarrow \quad Y_{\nu, \epsilon} \quad \text { is spacelike. }
$$

Set $\Xi=\left\{v \in \partial \widetilde{\Sigma}: \beta(1, v) \geq \delta_{4}\right\}$. There exists $\delta_{5}>0$ such that for $(r, v) \in\left[1-\delta_{5}, 1+\delta_{5}\right] \times \Xi$ we have $\operatorname{sgn}\left(\frac{\partial u_{\nu, \epsilon}}{\partial r}(r, v)\right)=\operatorname{sgn}\left(\frac{\partial u_{\nu, \epsilon}}{\partial r}(1, v)\right)$. Let $\delta_{6}=\min \left(\delta_{5}, K \epsilon / 8\right)$. For $(r, v) \in \Omega \equiv$ $\left(\left[1-\delta, 1-\delta_{6}\right] \cup\left[1+\delta_{6}, 1+\delta\right]\right) \times \partial \widetilde{\Sigma}$ the functions $u_{\nu, \epsilon}$ converge in $C^{k, \alpha} \subset C^{1}$ to $u$, it follows that decreasing $\nu_{0}$ if necessary spacelikeness of $Y_{\nu, \epsilon}$ for $\nu \leq \nu_{0} \leq K \epsilon / 8$ will hold on $\Omega$. On $\left[1-\delta_{6}, 1+\delta_{6}\right] \times \partial \widetilde{\Sigma}$ we have (recall that $u=0$ for $r \geq 1$ )

$$
\begin{aligned}
\frac{\partial u_{\nu, \epsilon}}{\partial r} & =I_{\nu, \epsilon}+I I_{\nu, \epsilon}, \\
I_{\nu, \epsilon} & =\int_{-\infty}^{\infty} \psi_{\nu}(r-s) \frac{\partial \widetilde{\widetilde{u}}}{\partial r}(s, v) d s, \\
I I_{\nu, \epsilon} & =-\int_{1}^{\infty} \psi_{\nu}(r-s) \frac{\partial \widetilde{\tilde{u}}}{\partial r}(s, v) d s
\end{aligned}
$$

(since $r \leq K \epsilon / 8$ and $\nu \leq K \epsilon / 8$ we have $\phi[X]_{\epsilon}(r)=1, \psi_{\nu}(r-s) \phi[X]_{\epsilon}(s)=\psi_{\nu}(r-s)$ ). For $v \notin \Xi$ spacelikeness of $Y_{\nu, \epsilon}$ has already been established; for $\nu \in \Xi$ the sign of $\frac{\partial \widetilde{\widetilde{u}}}{\partial r}$ is constant along the curves $v=$ const, so that

$$
\left|\frac{\partial u_{\nu, \epsilon}}{\partial r}\right| \leq\left|I_{\nu, \epsilon}\right|
$$

and since $I_{\nu, \epsilon}$ converges to $\frac{\partial \widetilde{\tilde{u}}_{\nu, \epsilon}}{\partial r}$ the inequality $(\mathbb{B . 9})$ follows from (B.7). 


\section{References}

[1] A. Ashtekar, B.C. Xanthopoulos, Jour. Math. Phys. 19, 2216 (1978).

[2] R. Bartnik, Commun. Math. Phys. 94, 155 (1984).

[3] R. Bartnik, Acta Math. 161, 145 (1988).

[4] J.M. Bardeen, B. Carter, S.W. Hawking, Commun. Math. Phys. 31, 161 (1973).

[5] R. Bartnik, P.T. Chruściel, N. O’Murchadha, Commun. Math. Phys. 130, 95 (1990).

[6] R. Beig, W. Simon, Proc. Roy. Soc. London A376, 333 (1981).

[7] E.M. Butterworth, J.R. Ipser, Astroph. Jour. 204, 200 (1976). Math.

[8] B. Carter, Black Hole Equilibrium States, in Black Holes, C. de Witt, B. de Witt, eds., Gordon \& Breach, New York, London, Paris, 1973.

[9] D. Christodoulou, N. O'Murchadha, Commun. Math. Phys. 80, 27 (1981).

[10] P.T. Chruściel, Jour. Math. Phys. 30, 2094 (1989).

[11] T. Damour, B. Schmidt, Jour. Math. Phys. 31, 10 (1990).

[12] D. Gilbarg, N. Trudinger, Elliptic Partial Differential Equations of Second Order, Springer Verlag, Berlin, 1983.

[13] R. Geroch, Jour. Math. Phys. 11, 437 (1970).

[14] R. Geroch, J. Hartle, Jour. Math. Phys. 23, 680 (1982).

[15] R. Geroch, G. Horowitz, Phys. Rev. Lett. 40, 203 (1978).

[16] J.B. Hartle, S.W. Hawking, Commun. Math. Phys. 26, 87 (1972).

[17] P. Hartman, Ordinary Differential Equations, John Hopkins University Press, Baltimore, 1973.

[18] S.W. Hawking, G.F.R. Ellis, The Large Scale Structure of Space-time, Cambridge University Press, Cambridge, 1973.

[19] I. Rácz, R.M. Wald, Extensions of Spacetimes with Killing Horizons, Class. Quant. Grav. (in press).

[20] W. Simon, R. Beig, Jour. Math. Phys. 24, 1163 (1983).

[21] D. Sudarsky, R.M. Wald, Phys. Rev. D46, 1453 (1992).

[22] R.M. Wald, General Relativity, University of Chicago Press, Chicago, 1984.

[23] M. Walker, Jour. Math. Phys. 11, 2280 (1970). 